\title{
Subvariedades Conformemente Planas de uma forma espacial
}

\section{Anarosa Alves Franco}

\author{
DISSERTAÇÃO APRESENTADA \\ $\mathrm{AO}$ \\ INSTITUTO DE MATEMÁTICA E ESTATÍSTICA \\ DA \\ UNIVERSIDADE DE SÃO PAULO \\ PARA OBTENÇÃO DO GRAU DE \\ MESTRE EM \\ MATEMÁTICA PURA
}

Área de Concentração: Geometria

Orientador: Prof. Dr. Antonio Carlos Asperti 


\title{
Subvariedades Conformemente Planas de uma forma espacial
}

\author{
Este exemplar corresponde à redação \\ final da dissertação devidamente corrigida \\ e defendida por Anarosa Alves Franco \\ e aprovada pela comissão julgadora.
}

São Paulo, 05 de agosto de 1994.

Banca examinadora:

- Prof. Dr. Antonio Carlos Asperti (orientador) - IME-USP

- Profa. Dra. Rosa Maria S. Barreiro Chaves - IME-USP

- Prof. Dr. Francesco Mercuri - IMECC-UNICAMP 
À memória de minha avó Profa. Maria José 


\section{Resumo}

Em termos de geometria riemanniana, uma variedade conformemente plana é uma variedade riemanniana tal que cada ponto tem uma vizinhança conformemente difeomorfa a um aberto do espaço euclidiano. Neste trabalho, nós estudamos alguns aspectos conhecidos da geometria local e global das variedades conformemente planas (assumidas como sendo) isometricamente imersas numa forma espacial. Sua parte principal (capítulo 2), descreve completamente a geometria e a topologia das hipersuperfícies conformemente planas compactas. Na última parte (capítulo 3), nós descrevemos alguns resultados algébricos, topológicos e geométricos sobre subvariedades conformemente planas em baixa codimensão. Como as formas espaciais de mesma dimensão são localmente conformemente difeomorfas, por simplicidade, nós sempre assumimos que o espaço ambiente é um espaço euclidiano. 


\section{Abstract}

In terms of Riemannian geometry, a conformally flat manifold is a Riemannian manifold such that each point has an open neighborhood conformally diffeomorphic to an open set of Euclidean space. In this work, we study some known aspects of the local and global geometry of conformally flat manifolds (assumed to be) isometrically immersed into a space form. The main part of this (chapter 2), completely describes the topology and geometry of compact conformally flat hypersurfaces. In the last part (chapter 3), we describe some algebraic, topological and geometric results on conformally flat submanifolds in low codimension. Since space forms of the same dimension are all locally conformally diffeomorphic, for simplicity, we always assume that the ambient space is an Euclidean space. 


\section{Agradecimentos}

A todas as pessoas que direta ou indiretamente contribuíram para que este trabalho chegasse a um fim, muito obrigada.

Agradecimentos especiais:

$\diamond$ Prof. Antonio Carlos Asperti, pela orientação, amizade e paciência;

$\diamond$ Profa. Maria Elisa G. G. de Oliveira, pela iniciação ao gosto pela pesquisa;

- Prof. Francesco Mercuri, pela disponibilidade na hora das dúvidas;

- Profa. Mary Lilian Lourenço, por me deixar à vontade na hora da escolha; - meus pais e minhas irmãs, pelo apoio incondicional em todos os momentos; $\diamond$ Leônidas, pela presença constante e ajuda incansável na confeç̧ão e edição das figuras que fazem parte deste trabalho. 


\section{Conteúdo}

Introdução

1 Preliminares $\quad 6$

1.1 Variedades Riemannianas - Curvaturas . . . . . . . . . . . . . 6

1.2 Imersões isométricas - As equações fundamentais . . . . . . . . . . . . 8

1.3 Variedades Conformemente Planas . . . . . . . . . . . . . . . . 10

1.4 Teoria de Morse - Alguns resultados . . . . . . . . . . . . . . 15

1.5 Colagem de alças em variedades diferenciáveis . . . . . . . . . . . 17

A Uma demonstração do Teorema de Weyl 19

2 Hipersuperfícies conformemente planas 23

2.1 Hipersuperfícies conformemente planas compactas . . . . . . . . . . . . 23

2.2 Hipersuperfícies conformemente planas sem pontos umbílicos . . . . . . . 31

2.3 Cirurgia Conforme . . . . . . . . . . . . . . . . . 40

2.4 Caracterização topológica das hipersuperfícies conformemente planas compactas 46 
2.5 Observações Finais . . . . . . . . . . . . . . . . . . . . 50

3 Subvariedades conformemente planas em baixa codimensão

3.1 Formas bilineares planas e exteriormente ortogonais . . . . . . . . . . . 52

3.2 Os teoremas de Moore e Moore-Morvan . . . . . . . . . . . . . . . . 57

Referências Bibliográficas 


\section{Introdução}

Uma variedade riemanniana (localmente) conformemente plana, é uma variedade em que todo ponto tem uma vizinhança que é conformemente difeomorfa a um aberto do espaço euclidiano de mesma dimensão. É bastante conhecido que essa condição local pode ser transformada numa condição tensorial (pontual) dada, quando a dimensão da variedade é $n \geq 4$ pelo anulamento do tensor de Weyl. Uma condição extra que facilita o estudo de uma variedade conformemente plana é supor que possa ser isometricamente imersa como hipersuperfície, ou em codimensão baixa, em um espaço euclidiano.

Apresentamos neste trabalho uma coletânea de resultados que caracterizam as variedades conformemente planas, principalmente as que podem ser imersas em codimensão relativamente baixa.

O artigo que serviu como base inicial e motivação aos nossos estudos foi Compact Conformally Flat Hipersurfaces de M. do Carmo, M. Dajczer e F. Mercuri, [C-D-M]. Neste artigo encontramos a descrição completa das hipersuperfícies conformemente planas compactas $M^{n}$, para $n \geq 4$, imersas numa variedade riemanniana completa, simplesmente conexa e de curvatura seccional constante, $M^{n+1}(c)$.

Depois disso, entramos em contato com a resenha de F. Mercuri, Conformally Flat Immersions, [M], e fomos levados de forma natural ao artigo de J.D. Moore, [Moo1], onde sào dadas caracterizações topológica e algébrica para subvariedades conformemente planas de codimensão maior que $1(\leq n-3)$, e ao artigo de Moore-Morvan, [Moo-Mor], onde é dada uma caracterização geométrica para codimensão menor ou igual a 4. Observamos que a descrição topológica de subvariedades conformemente planas refere-se apenas ao caso de subvariedades compactas.

Os três artigos acima mencionados são completamente descritos nos capítulos 2 e 3 deste trabalho, que se constitui de três capítulos. A seguir apresentamos o conteúdo de cada um 
deles.

No Capítulo 1 procuramos dar as definições e os resultados básicos que serão utilizados nos capítulos seguintes. Assim é que, neste capítulo, muitos teoremas são enunciados e poucos demonstrados. Dentre estes últimos, apresentamos na seção 1.2 uma demonstração para o seguinte resultado de Kulkarni: Seja $M^{n}, n \geq 4$, uma variedade riemanniana. Então $M^{n} e ́$ conformemente plana se, e somente se, para todo $p \in M$ e toda quádrupla $\left\{e_{1}, e_{2}, e_{3}, e_{4}\right\}$ de vetores ortonormais de $T_{p} M$, tem-se $k\left(e_{1}, e_{2}\right)+k\left(e_{3}, e_{4}\right)=k\left(e_{1}, e_{3}\right)+k\left(e_{2}, e_{4}\right)$, onde $k\left(e_{i}, e_{j}\right)$ é a curvatura seccional relativamente ao plano gerado por $\left\{e_{i}, e_{j}\right\}$; no Apêndice A damos uma demonstração, usando geometria de Lorentz, do Teorema de Weyl: Seja $M^{n}, n \geq 4$, uma variedade riemanniana. Então $M^{n}$ é conformemente plana se, e somente se, $C \equiv 0$, onde $C$ é o tensor de Weyl definido em (1.4). Tal demonstração contém uma demonstração para o seguinte resultado de Asperti-Dajczer, [A-D]: Uma variedade riemanniana $M^{n}, n \geq 3$, é conformemente plana se, e somente se, existe uma imersão isométrica local $f: M^{n} \rightarrow V^{n+1}$. Além disso, se $M$ é simplesmente conexa, a imersão é definida em toda $M$. Aqui $V^{n+1}$ é o cone de luz definido em (1.5).

O Capítulo 2 é a parte principal do nosso trabalho. Nele apresentamos os resultados de [C-D-M], os quais descrevemos a seguir. Sendo $M^{n}$ uma hipersuperfície conformemente plana compacta, $n \geq 4$, imersa numa variedade riemanniana completa, simplesmente conexa e de curvatura seccional constante, e $U$ o seu conjunto de pontos umbílicos, o Teorema 2.1.2 descreve a estrutura das componentes conexas de $M-U$ e de $U$ : (i) cada componente conexa $D$ do conjunto $M-U$ admite uma folheação de codimensão 1 por $(n-1)$-esferas; em particular $D e ́$ difeomorfo a $S^{n-1} \times(a, b)$; (ii) cada componente conexa de $U$ é um ponto, uma $(n-1)$-esfera ou um conjunto $n$-dimensional umbilico limitado por pontos ou pela união de $(n-1)$-esferas, tal que a união de quaisquer duas delas tenha, no máximo, um ponto em comum. Se $U=\emptyset$, apresentamos também a descrição de $M^{n}$ como a envolvente de uma família de $n$-esferas a um parâmetro (Proposição 2.13). Tal resultado também pode ser encontrado em [D] pag.127, Teo. 7.18. Mais adiante neste capítulo, é definido cirurgia conforme e é demonstrado quando é possível realizá-la (veja Proposição 2.3.3). Este fato é utilizado na demonstração do teorema final do Capítulo 2, Teorema 2.4.1, o qual caracteriza as variedades diferenciáveis compactas $M^{n}, n \geq 4$, que podem ser imersas como hipersuperfícies conformemente planas de um espaço euclidiano: $M^{n}$ deve ser difeomorfa a uma $n$-esfera $\operatorname{com} b$ alças, $S_{b}^{n}$, onde $b$ é o primeiro número 
de Betti de $M^{n}$.

O Capítulo 3 é um apanhado dos artigos [Moo1] e [Moo-Mor], onde apresentamos as seguintes caracterizações para subvariedades conformemente planas em codimensão baixa:

a) (caracterização algébrica) Seja $f: M^{n} \rightarrow \mathbb{R}^{n+r}$ uma imersão isométrica, $r \leq n-3$. Se $M$ é conformemente plana, para todo $p \in M$ existe um subespaço umbílico $U \subseteq T_{p} M$ tal que $\operatorname{dim} U \geq n-r$;

b) (caracterização topológica) Seja $M^{n}$ uma variedade compacta e $f: M^{n} \rightarrow \mathbb{R}^{n+r}$ uma $i$ mersão isométrica, $r \leq n-3$. Então $M^{n}$ tem o tipo homotópico de um $C W$ sem células de dimensão $k$ para $r<k<n-r$. Em particular, para $k$ nessas condições, os grupos de homologia $H_{k}\left(M^{n}, \mathbb{Z}\right)$ são nulos;

c) (caracterização geométrica) Seja $f: M^{n} \rightarrow \mathbb{R}^{n+r}$ uma imersão isométrica com $r \leq$ $\min \{4, n-3\}$. Então $M^{n}$ é conformemente plana se e somente se $f$ é quase umbítica.

Convém mencionarmos que, para a leitura desses artigos, foi necessário entrarmos em contato com as formas exteriormente ortogonais de E. Cartan, [Ca].

Pode-se notar que a sequência apresentada seguiu bem de perto a utilizada na resenha de F. Mercuri citada acima, até o início de sua seção 4. Nessa resenha são ainda descritos alguns resultados sobre subvariedades conformemente planas em codimensão 2, contidos nos seguintes artigos de M.H. Noronha: Conformally flat immersion in codimension two, Geometria Dedicata, 23, (1987), 115-130 e Conformally flat immersions and the flatness of the normal connection, Pac. J. of Math., 138, 145-150. Por motivos de espaço e tempo, o estudo desses últimos artigos não foram incluídos neste trabalho. 


\section{Capítulo 1}

\section{Preliminares}

Neste capítulo reuniremos algumas definições e resultados básicos da Geometria Riemanniana, Teoria de Morse e introduziremos alguns conceitos básicos da teoria das variedades conformemente planas que serão utilizados no decorrer do trabalho. Além disso pretendemos fixar a notação e, portanto, poucas demonstrações serão apresentadas.

\subsection{Variedades Riemannianas - Curvaturas}

Seja $\left(M^{n},<,>\right), n \geq 2$, uma variedade riemanniana n-dimensional, isto é, $M^{n}$ é uma variedade diferenciável (Hausdorff e com base enumerável) de classe $C^{\infty}$ e $<,>$ é uma métrica riemanniana em $M^{n}$. Chamaremos de $\mathcal{X}(M)$ o conjunto dos campos de vetores de classe $C^{\infty}$ tangentes a $M$ e de $\mathcal{D}(M)$ o anel das funções reais, de mesma classe, definidas em $M$. Indicaremos ainda por $\nabla$ a conexão riemanniana ou de Levi-Civita de $M^{n}$. 
A curvatura $R$ de uma variedade riemanniana $M^{n}$ é a correspondência que associa a cada par $X, Y$ em $\mathcal{X}(M)$ uma aplicação $R(X, Y): \mathcal{X}(M) \rightarrow \mathcal{X}(M)$ dada por

$$
R(X, Y) Z=\nabla_{X} \nabla_{Y} Z-\nabla_{Y} \nabla_{X} Z-\nabla_{[X, Y]} Z, Z \in \mathcal{X}(M)
$$

onde $[X, Y]$ é o colchete de Lie dos campos $X, Y$. O tensor de curvatura de $M$ é definido por

$$
R(X, Y, Z, W):=<R(X, Y) Z, W>, X, Y, Z, W \in \mathcal{X}(M)
$$

Sejam agora $p \in M$ e $\sigma \subset T_{p} M$ um subespaço bidimensional do espaço $T_{p} M$ tangente a $M$ em $p$. Se $\{x, y\}$ é uma base qualquer de $\sigma$, o número $k(\sigma)(k(x, y))$ dado por

$$
k(\sigma):=\frac{R(x, y, y, x)}{|x \wedge y|^{2}},|x \wedge y|^{2}=|x|^{2}|y|^{2}-<x, y>^{2}
$$

não depende da base $\{x, y\}$ tomada e é denominada curvatura seccional de $\sigma$ em $p$. Observamos que o conhecimento de $k(\sigma)$, para todo $\sigma \subset T_{p} M$, determina o tensor $R$ em $p$ (veja Lema 3.3 pag. 94 de [C]). Lembramos que uma variedade riemanniana $M$ tem curvatura seccional constante $c$ quando $k(\sigma)=c$, para todo $\sigma \subset T_{p} M$ e todo $p \in M$. Para $X, Y$ em $\mathcal{X}(M)$, definamos $X \wedge Y: \mathcal{X}(M) \rightarrow \mathcal{X}(M)$ por

$$
X \wedge Y:=<X, \cdot>Y-<Y, \cdot>X .
$$

O fato que a curvatura seccional determina o tensor de curvatura pode ser diretamente usado para se mostrar que $M^{n}$ tem curvatura seccional constante c se, e somente se, $R(X, Y)=$ $-c(X \wedge Y)$, para $X, Y \in \mathcal{X}(M)$. É um fato bem conhecido (veja Teorema 4.1, pag. 163 de [C]) que, se $M^{n} e ́$ uma variedade riemanniana completa com curvatura seccional constante $c$, então o seu recobrimento universal $\tilde{M}^{n}$, com a métrica do recobrimento, é isométrico a:

(i) $\mathbb{R}^{n}$, se $c=0$;

(ii) Esfera $S^{n}\left(\frac{1}{\sqrt{c}}\right):=\left\{y \in \mathbb{R}^{n+1}:\|y\|^{2}=\frac{1}{c}\right\}$, se $c>0$;

(iii) Espaço hiperbólico $H^{n}(c)=\left(B^{n}, g\right)$, se $c<0$, onde

$$
B^{n}=B^{n}\left(\frac{1}{\sqrt{-c}}\right)=\left\{x \in \mathbb{R}^{n}:\|x\|^{2}<\frac{1}{-c}\right\},
$$

e g é a métrica riemanniana definida em $x \in B^{n}$ por

$$
g_{x}:=\frac{4}{\left(1+c\|x\|^{2}\right)^{2}}<,>
$$


sendo $<,>$ o produto interno usual de $\mathbb{R}^{n}$.

Seja ainda $p \in M$ e seja $x$ um vetor unitário de $T_{p} M$. Tomemos uma base ortonormal $\left\{e_{1}, \ldots, e_{n}\right\}$ tal que $e_{1}=x$. Então

$$
\operatorname{Ric}_{p}(x):=\sum_{i=2}^{n} R\left(x, e_{i}, e_{i}, x\right)=\sum_{i=2}^{n} k\left(x, e_{i}\right)
$$

não depende de $e_{2}, \ldots, e_{n}$ e é denominada curvatura de Ricci de $M$ em $p$ na direção de $x$. Do mesmo modo,

$$
S(p):=\sum_{i=1}^{n} \operatorname{Ric} c_{p}\left(e_{i}\right)=\sum_{i \neq j} k\left(e_{i}, e_{j}\right)
$$

não depende da base ortonormal $\left\{e_{1}, \ldots, e_{n}\right\}$ de $T_{p} M$ tomada e é denominada curvatura escalar de $M$ em $p$. Finalmente definimos o tensor de Ricci de $M$ em $p$ por

$$
\operatorname{Ricc}(x, y):=\text { traço da aplicação } z \mapsto R(z, x) y,
$$

$x, y, z \in T_{p} M$. Observamos que $\operatorname{Ric}_{p}(x)=\operatorname{Ricc}(x, x)$.

\subsection{Imersões isométricas - As equações fundamentais}

Sejam $M^{n}$ e $\bar{M}^{n+r}, r \geq 1$, variedades riemannianas com as respectivas conexões $\nabla$ e $\bar{\nabla}$. Se $f: M \rightarrow \bar{M}$ é uma imersão isométrica, denotamos por $\nu M$ o fibrado normal, e por $\nabla^{\perp}$ a conexão normal da imersão. Se $X, Y \in \mathcal{X}(M)$ e se $\dot{\xi} \in \mathcal{X}(M)^{\perp}$ (=seç̧ões de $\nu M$ ), temos as fórmulas de Gauss e de Weingarten

$$
\begin{gathered}
\bar{\nabla}_{X} Y=\nabla_{X} Y+\alpha(X, Y), \\
\bar{\nabla}_{X} \xi=-A_{\xi} X+\bar{\nabla}_{X}^{\perp} \xi,
\end{gathered}
$$

respectivamente, onde $\alpha: T M \times T M \rightarrow \nu M$ é a segunda forma fundamental de $f$ e $A_{\xi}: T M \rightarrow T M$ é o operador de Weingarten na direção $\xi$. É bem sabido que $\alpha$ é ponto a ponto bilinear e simétrica, que $A_{\xi}$ é endomorfismo simétrico de $T M$ e que $\langle\alpha(X, Y), \xi\rangle=$ $=<A_{\xi} X, Y>$, onde $<,>$ denota tanto a métrica de $M$ quanto a de $\bar{M}$. 
Utilizando as fórmulas de Gauss e Weingarten, chegamos às equações fundamentais de uma imersão isométrica $f$. Para este trabalho é conveniente escrevê-las no caso em que $\bar{M}=\bar{M}(c)$ é uma variedade riemanniana com curvatura seccional constante igual a $c$.

1.2.1 Equações fundamentais de uma imersão isométrica $f: M \rightarrow \bar{M}(c)$

Para quaisquer $X, Y, Z, W \in \mathcal{X}(M), \xi, \eta \in \mathcal{X}(M)^{\perp}$, temos:

Equação de Gauss: $\quad R(X, Y, Z, W)=c(<Y, Z><X, W>-<X, Z><Y, W>)$ $+<\alpha(X, W), \alpha(Y, Z)>-<\alpha(X, Z), \alpha(Y, W)>$;

Equação de Codazzi: $\left(\nabla_{X}^{\frac{1}{}} \alpha\right)(Y, Z)=\left(\nabla_{Y}^{\perp} \alpha\right)(X, Z)$;

Equação de Ricci: $R^{\perp}(X, Y) \xi=\alpha\left(X, A_{\xi} Y\right)-\alpha\left(A_{\xi} X, Y\right)$,

onde $\nabla^{\perp} \alpha$ é a derivação covariante normal estendida para agir em tensores e $R^{\perp}$ é a curvatura normal da imersão $f$, isto é,

$$
\begin{aligned}
\left(\nabla_{X}^{\perp} \alpha\right)(Y, Z) & :=\nabla_{X}^{\perp}(\alpha(Y, Z))-\alpha\left(\nabla_{X} Y, Z\right)-\alpha\left(Y, \nabla_{X} Z\right), \\
R^{\perp}(X, Y) \xi & :=\nabla_{X}^{\perp} \nabla_{Y}^{\perp} \xi-\nabla_{Y}^{\perp} \nabla_{X}^{\perp} \xi-\nabla_{[X, Y]}^{\perp} \xi
\end{aligned}
$$

As equações acima são básicas no sentido que elas determinam a geometria da imersão em vista do teorema a seguir

Teorema 1.2.2 (Teorema fundamental da teoria das subvariedades) Seja $M^{n}$ uma variedade riemanniana, $E$ um $r$-dimensional fibrado vetorial riemanniano sobre $M$ com conexão métrica $\nabla^{\prime}$ e respectiva curvatura $R^{\prime}$. Seja $\alpha^{\prime}: T M \oplus T M \rightarrow E$ aplicação ponto a ponto bilinear simétrica e, para cada seç̧ão $\varepsilon$ de $E$, seja $A_{\varepsilon}^{\prime}$ o endomorfismo de $T M$ definido de maneira natural por $\alpha^{\prime}$. Suponha que para algum real c, os dados $\alpha^{\prime}, R^{\prime}, A_{\varepsilon}^{\prime}$ satisfazem formalmente as equações 1.2.1. Então, existe uma imersão isométrica localmente definida $f$ de $M$ em $\bar{Q}^{n+r}(c)\left(=\mathbb{R}^{n+r}, S^{n+r}\left(\frac{1}{\sqrt{c}}\right)\right.$ ou $\left.H^{n}(c)\right)$, com $\nu M$ isométrico a $E$ e com $\nabla^{\perp} e \alpha$ correspondendo a $\nabla^{\prime}$ e $\alpha^{\prime}$ através dessa isometria. Além disso, a imersão é única, a menos de isometrias de $\bar{Q}^{n+r}(c)$, e será globalmente definida se $M$ for simplesmente conexa.

Para uma demonstração do teorema acima no caso $c=0$, veja teorema 1.1 pag. 7 em [D] e no caso $c$ qualquer veja $[\mathrm{S}]$, vol. IV, Cap 7 . 


\subsection{Variedades Conformemente Planas}

Em geral, uma variedade conformemente plana é uma variedade diferenciável munida de um atlas cujas mudanças de coordenadas são difeomorfismos conforme entre abertos do espaço euclidiano correspondente. Em termos de geometria riemanniana, temos a definição seguinte, a qual usaremos neste trabalho e que, claramente, implica a noção acima.

Definição 1.3.1 Uma variedade riemanniana $M^{n}$ é (localmente) conformemente plana quando cada ponto de $M$ tem uma vizinhança que é conformemente difeomorfa a um aberto do espaço euclidiano $\mathbb{R}^{n}$.

As variedades de curvatura constante definidas na Seção 1.1 são conformemente planas. Todas as superfícies (ou variedades riemannianas de dimensão 2) também são conformemente planas como consequência da existência de parâmetros isotérmicos (veja [S], vol IV, pag.445). Para caracterizar as variedades conformemente planas de dimensão $n \geq 3$, introduziremos alguns conceitos. Primeiramente definimos o operador bilinear simétrico (ou tensor $(2,0)$ )

$L: \mathcal{X}(M) \times \mathcal{X}(M) \rightarrow \mathcal{D}(M)$ por

$$
L(X, Y):=\frac{1}{n-2}\left[\operatorname{Ricc}(X, Y)-\frac{S}{2(n-1)}<X, Y>\right] X, Y \in \mathcal{X}(M) .
$$

O tensor de Weyl é o $(3,1)$-tensor $C$ em $\mathcal{X}(M)$ definido por

$$
\begin{aligned}
<C(X, Y) Z, W>= & R(X, Y, Z, W)-\{L(X, W)<Y, Z>-L(X, Z)<Y, W>+ \\
& +L(Y, Z)<X, W>-L(Y, W)<X, Z>\} .
\end{aligned}
$$

Temos a seguinte caracterização pontual de variedades conformemente planas,

Teorema 1.3.2 (Weyl) Uma variedade riemanniana $M^{n}, n \geq 3$, é conformemente plana se, e somente se, valem

(i) $C \equiv 0$ em $M$;

(ii) $\left(\nabla_{X} L\right)(Y, Z)=\left(\nabla_{Y} L\right)(X, Z), \quad \forall X, Y, Z \in \mathcal{X}(M)$.

Além disso, se $n \geq 4$, (i) implica (ii). 
A demonstração completa do Teorema de Weyl pode ser encontrada em [B] e [E]; veja também apêndice deste capítulo. Faremos uso, no Capítulo 2 deste trabalho, do seguinte teorema clássico de $\mathrm{N}$. Kuiper [K], que vale em qualquer dimensão $n \geq 2$.

Teorema 1.3.3 (Kuiper) Seja $M^{n}$ uma variedade conformemente plana compacta, conexa e simplesmente conexa. Então $M^{n}$ é conformemente difeomorfa à esfera unitária euclidiana $S^{n}$.

Passaremos agora a descrever uma caracterização de variedades conformemente planas em termos de geometria de Lorentz. Conforme veremos, essa caracterização tem como consequência uma demonstração alternativa do Teorema 1.3.3. Seja $\mathbb{L}^{n+2}$ o espaço de Lorentz de dimensão $n+2$, isto é, $\mathbb{L}^{n+2}=\left(\mathbb{R}^{n+2},(),\right)$, onde $($,$) denota a aplicação bilinear simétrica não$ degenerada (métrica indefinida) de $\mathbb{R}^{n+2}$ dada por

$$
(x, y)=\sum_{i=1}^{n+1} x_{i} y_{i}-x_{n+2} y_{n+2}, \quad x=\left(x_{1}, \ldots, x_{n+2}\right), y=\left(y_{1}, \ldots, y_{n+2}\right) \in \mathbb{R}^{n+2}
$$

Se $\left\{e_{1}, \ldots, e_{n+2}\right\}$ é base canônica de $\mathbb{R}^{n+2}$, então $\left(e_{i}, e_{j}\right)=\delta_{i j},\left(e_{i}, e_{n+2}\right)=0,1 \leq i, j \leq n+1$, e $\left(e_{n+2}, e_{n+2}\right)=-1$. O cone de luz em $\mathbb{L}^{n+2}$ é definido por

$$
V^{n+1}=\left\{x \in \mathbb{L}^{n+2}:(x, x)=0, x_{n+2}>0\right\} .
$$

É fácil ver que os vetores $v_{1}(x)=\left(1,0, \ldots, 0, \frac{x_{1}}{x_{n+2}}\right), \ldots, v_{n+1}(x)=\left(0, \ldots, 0,1, \frac{x_{n+1}}{x_{n+2}}\right)$ formam uma base do espaço tangente a $V^{n+1}$ em $x$. Observamos que $x=\sum_{i=1}^{n+1} x_{i} v_{i}(x)$ é, ele próprio, tangente ao cone em $x$. Temos que

$$
\left(v_{i}(x), v_{j}(x)\right)=-\frac{x_{i} x_{j}}{x_{n+2}^{2}}, \quad(x, x)=0,
$$

donde a métrica $($, ) restrita ao cone é semidefinida e as direções de norma nula são as geratrizes do cone.

Teorema 1.3.4 Uma variedade riemanniana $M^{n}, n \geq 3$, é conformemente plana se, e somente se, existe uma imersão isométrica local $f: M^{n} \rightarrow V^{n+1}$. Além disso, se $M$ é simplesmente conexa, a imersão é definida em toda $M$. 
O teorema acima encontra-se demonstrado em [A-D] e [D]. No Apêndice A apresentaremos uma demonstração quase completa do Teorema 1.3.2 (Weyl) usando a teoria das subvariedades de um espaço de Lorentz $\mathbb{L}^{n}$, e nessa demonstração estará contida uma demonstração do Teorema 1.3.4. Neste capítulo apresentaremos a seguinte consequência do teorema acima.

Demonstração do teorema 1.3.3 (Kuiper) Se $n=2$ segue diretamente do teorema de Gauss-Bonet. Se $n \geq 2$, seja $f: M^{n} \rightarrow V^{n+1}$ a imersão isométrica global dada pelo Teorema 1.3.4. Como $(f(p), f(p))=0, \forall p \in M$, temos que $0=X_{p}(f, f)=\left(d f_{p} X, f(p)\right)$ para todo $p \in M$ e $X \in T_{p} M$. Temos ainda que $\varphi: M^{n} \rightarrow \mathbb{R}$ dada por $\varphi(p)=\left(f(p), e_{n+2}\right)$ é sempre diferente de zero, uma vez que, estando $f(p)$ em $V^{n+1}$, a sua $(n+2)$-ésima coordenada é sempre positiva.

Seja $\bar{S}^{n}=\left\{x \in V^{n+1}:\left(x, e_{n+2}\right)=-1\right\}$ e observemos que $($,$) restrito a \bar{S}^{n}$ o torna isométrico à esfera unitária euclidiana $S^{n}$. Definamos então $F: M^{n} \rightarrow \bar{S}^{n} \subset V^{n+1}$ por $F(p)=-\frac{f(p)}{\varphi(p)}$. Temos que $(F, F)=0$ e, consequentemente, $\left(d F_{p} X, F(p)\right)=0, \quad X \in T_{p} M$. Segue-se daí que

$$
\left(d F_{p} X, d F_{p} Y\right)=\frac{1}{\varphi^{2}(p)}\left(d f_{p} X, d f_{p} Y\right)=\frac{1}{\varphi^{2}(p)}<X, Y>, \quad X, Y \in T_{p} M
$$

Assim, $F$ define uma imersão conforme (global), $\tilde{F}$, da variedade riemanniana $M^{n}$ na esfera euclidiana de mesma dimensão n. Então $\tilde{F}: M^{n} \rightarrow S^{n}$ é um difeomorfismo local globalmente definido. Como $M$ é compacta, $\tilde{F}$ é uma aplicação de recobrimento e, como $S^{n}$ é simplesmente conexa, $\tilde{F}$ é difeomorfismo conforme.

Para finalizarmos esta seção, vamos apresentar uma caracterização das variedades conformemente planas com dimensão $n \geq 4$ em termos de curvaturas seccionais, devida a Kulkarni [Kul-2] e que será utilizada no decorrer do trabalho. Antes disso, precisamos de algumas definições.

Definição 1.3.5 (Veja [Kul-2]) Seja $\sigma$ um 2-plano de $T_{p} M$ e seja $\left\{e_{1}, \ldots, e_{n}\right\}$ base ortonormal de $T_{p} M$ tal que $\sigma=\left[e_{1}, e_{2}\right]$. Denotemos $k\left(e_{i}, e_{j}\right)$ por $k_{i j}, i \neq j$. Chamamos de curvatura seccional conforme do 2-plano $\sigma$ ao número real

$$
k_{\text {con }}(\sigma):=k(\sigma)-\frac{1}{n-2}\left\{\sum_{i \neq 1} k_{1 i}+\sum_{i \neq 2} k_{2 i}\right\}+\frac{S}{(n-1)(n-2)} .
$$


O tensor de Ricci correspondente, chamado tensor de Ricci conforme é dado por

$$
\operatorname{Ricc}_{c o n}(X, Y):=\text { traço da aplicação } Z \mapsto C(Z, X) Y
$$

$A$ curvatura escalar conforme é dada por

$$
S_{\text {con }}:=\text { traço Ricc con }_{\text {. }}
$$

Observemos que $k_{\text {con }}\left(e_{i}, e_{j}\right)=k\left(e_{i}, e_{j}\right)-L\left(e_{i}, e_{i}\right)-L\left(e_{j}, e_{j}\right)=<C\left(e_{i}, e_{j}\right) e_{j}, e_{i}>$ e que $S_{c o n}=\sum_{i \neq j} k_{c o n}\left(e_{i}, e_{j}\right)$.

Proposição 1.3.6 Ricc con $\equiv 0$ e consequentemente $S_{\text {con }} \equiv 0$.

Demonstração: Basta demonstrarmos que $\operatorname{Ricc}_{c o n}\left(e_{1}, e_{1}\right)=0$ pois $\operatorname{Ricc}_{\text {con }}$ é bilinear simétrica.

De fato,

$\operatorname{Ricc}_{c o n}\left(e_{1}, e_{1}\right)=\operatorname{Ricc}\left(e_{1}, e_{1}\right)-\frac{1}{n-2}\left[\sum_{i>1}\left(\operatorname{Ricc}\left(e_{1}, e_{1}\right)+\operatorname{Ricc}\left(e_{i}, e_{i}\right)\right)\right]+\frac{S}{(n-1)(n-2)}(n-1)=$ $=\operatorname{Ricc}\left(e_{1}, e_{1}\right)-\frac{1}{n-2}\left[(n-1) \operatorname{Ricc}\left(e_{1}, e_{1}\right)+S-\operatorname{Ricc}\left(e_{1}, e_{1}\right)\right]+\frac{S}{n-2}=0$.

Finalmente podemos apresentar o

Teorema 1.3.7 As seguintes condições sobre uma variedade riemanniana $M^{n}$, com $n \geq 4$, são equivalentes

(i) $M$ é conformemente plana;

(ii) $C \equiv 0$ em $M$;

(iii)para todo ponto $p \in M$ e para toda quádrupla $\left\{e_{1}, e_{2}, e_{3}, e_{4}\right\}$ de vetores ortonormais em $T_{p} M$, tem-se

$$
k_{12}+k_{34}=k_{13}+k_{24} .
$$

Demonstração: A equivalência entre (i) e (ii) é o Teorema 1.3.2 (Weyl) acima e uma demonstração será apresentada no Apêndice A. Faremos aqui a demonstração da equivalência (ii) e (iii). 
Supondo primeiramente que $C \equiv 0$ temos, pela definição de $C$ em (1.4) ,que $R(X, Y, Z, W)=L(X, W)<Y, Z>-L(X, Z)<Y, W>+L(Y, Z)<X, W>-L(Y, W)<X, Z>$ para todos $X, Y, Z, W \in \mathcal{X}(M)$. Segue-se daí que, se $X_{1}, X_{2}, X_{3}, X_{4}$ são quaisquer campos ortonormais,

$$
k\left(X_{i}, X_{j}\right)=R\left(X_{i}, X_{j}, X_{j}, X_{i}\right)=L\left(X_{i}, X_{i}\right)+L\left(X_{j}, X_{j}\right), \quad 1 \leq i \neq j \leq 4
$$

Desta igualdade conclui-se que vale (iii).

Supondo agora (iii), vamos começar mostrando que $k_{\text {con }} \equiv 0$ em $M$. Seja $\sigma$ um 2-plano do espaço $T_{p} M, p \in M$, e seja $\left\{e_{1}, \ldots, e_{n}\right\}$ base ortonormal de $T_{p} M, \operatorname{com} \sigma=\left[e_{1}, e_{2}\right]$. Temos $k_{\text {con }}\left(e_{i}, e_{j}\right)=k_{\text {con }}\left(e_{i}, e_{l}\right)$ para todos $i=1, \ldots, n$ e $j, l \neq i$. De fato, por exemplo

$$
\begin{aligned}
k_{\text {con }}\left(e_{1}, e_{2}\right)-k_{\text {con }}\left(e_{1}, e_{3}\right) & =k_{12}-k_{13}-\frac{1}{n-2}\left(\sum_{i \neq 1} k_{1 i}+\sum_{j \neq 2} k_{2 j}\right)+\frac{1}{n-2}\left(\sum_{l \neq 1} k_{1 l}+\sum_{l \neq 3} k_{3 l}\right) \\
& =k_{12}-k_{13}-\frac{1}{n-2}\left(\sum_{i \neq 2} k_{2 i}-\sum_{j \neq 3} k_{3 j}\right) \\
& =k_{12}-k_{13}-\frac{1}{n-2}\left(\left(k_{12}-k_{13}\right)+\left(k_{24}-k_{34}\right)+\ldots+\left(k_{2 n}-k_{3 n}\right)\right) \\
& =k_{12}-k_{13}-\frac{1}{n-2}\left\{(n-2)\left(k_{12}-k_{13}\right)\right\}=0,
\end{aligned}
$$

onde usamos (iii) para ver que $k_{12}-k_{13}=k_{24}-k_{34}=\ldots=k_{2 n}-k_{3 n}$. Segue-se daí que, $\forall i, j, l, m$ dois a dois distintos,

$$
k_{\text {con }}\left(e_{i}, e_{j}\right)-k_{\text {con }}\left(e_{l}, e_{m}\right)=\left[k_{\text {con }}\left(e_{i}, e_{j}\right)-k_{\text {con }}\left(e_{i}, e_{l}\right)\right]+\left[k_{\text {con }}\left(e_{l}, e_{i}\right)-k_{\text {con }}\left(e_{l}, e_{m}\right)\right]=0
$$

isto é, $k_{\text {con }}\left(e_{i}, e_{j}\right)=a \quad \forall 1 \leq i \neq j \leq n$. Mas,

$$
0 \equiv S_{c o n}=\sum_{i \neq j} k_{c o n}\left(e_{i}, e_{j}\right)=n(n-1) a,
$$

logo $a=0$, portanto $k_{c o n}(\sigma)=k_{c o n}\left(e_{1}, e_{2}\right)=0$, o que prova a afirmação feita.

Como $k_{\text {con }}(X, Y)=k(X, Y)-L(X, X)-L(Y, Y)$ para qualquer par ortonormal $(X, Y)$, temos

$$
k(X, Y)=L(X, X)+L(Y, Y), X \text { e } Y \text { ortonormais. }
$$


Para concluir, definimos o $(4,0)$-tensor $R^{\prime}$ em $M$

$$
R^{\prime}(X, Y, Z, W):=R(X, Y, Z, W)-<C(X, Y) Z, W>
$$

e observamos, após cálculos diretos, que $R^{\prime}$ satisfaz as mesmas propriedades fundamentais do tensor de curvatura $R$ (veja [C], pag. 91). Observamos também que a curvatura seccional $k^{\prime}(\sigma)$ de $R^{\prime}$ (veja $(1.1),(1.2)$ ) coincide com $k(\sigma)$, devido a (1.6). Como a curvatura seccional determina o tensor de curvatura, temos $R^{\prime} \equiv R$ e, portanto $C \equiv 0$ em $M$.

1.3.8 Observação: Decorre da demonstração acima que $k_{\text {con }} \equiv 0$ é equivalente a $C \equiv 0$ e, portanto, é equivalente a $M^{n}$ ser conformemente plana, para $n \geq 4$. Além disso, (1.6) é equivalente a $C \equiv 0$, fato que será utilizado no Capítulo 3 .

\subsection{Teoria de Morse - Alguns resultados}

Seja $g: M^{n} \rightarrow \mathbb{R}$ uma função diferenciável. Um ponto $p \in M$ é chamado um ponto crítico de $g$ em $p$ se a aplicação induzida $d g_{p}: T_{p} M \rightarrow T_{g(p)} \mathbb{R}$ é nula. Se $\left(U,\left(x_{1}, \ldots, x_{n}\right)\right)$ é um sistema de coordenadas em $p$, isso é equivalente a

$$
\frac{\partial g}{\partial x_{1}}=\ldots=\frac{\partial g}{\partial x_{n}}=0
$$

Se $p \in M$ é um ponto crítico de $g$, a matriz dada por

$$
d^{2} G=\left(\frac{\partial^{2} g}{\partial x_{i} \partial x_{j}}(p)\right)
$$

define uma aplicação bilinear simétrica denominada o hessiano de $g$ em $p$. Além disso, o ponto $p$ é chamado não degenerado se, e somente se, essa matriz é não singular.

$\mathrm{O}$ índice de uma aplicação bilinear $H$ sobre um espaço vetorial $V$ é a máxima dimensão de um subespaço de $V$ onde $H$ é negativa definida. Ao índice de $d^{2} G$ sobre $T_{p} M$ chamaremos apenas de índice de $g$ em $p$. 
Dois espaços $X$ e $Y$ têm o mesmo tipo homotópico se existem aplicações contínuas $\varphi_{1}: X \rightarrow Y$ e $\varphi_{2}: Y \rightarrow X$ tais que $\varphi_{1} \circ \varphi_{2} \simeq i d_{Y}: Y \rightarrow Y$ e $\varphi_{2} \circ \varphi_{1} \simeq i d_{X}: X \rightarrow X$, onde $i d_{X}$ e $i d_{Y}$ são as aplicações identidade de $X$ e $Y$, respectivamente, e o símbolo $\simeq$ significa a existência de homotopia entre as aplicações correspondentes.

Teorema 1.4.1 (Teorema fundamental da teoria de Morse) Se g é uma função diferenciável a valores reais sobre uma variedade $M$ sem pontos críticos degenerados, e se cada conjunto $M^{c}=\{p \in M: g(p) \leq c\}$, para $c \in \mathbb{R}$, é compacto, então $M$ tem o tipo homotópico de um $C W$, com uma célula de dimensão $k$ para cada ponto crítico de índice $k$.

A demonstração do teorema acima encontra-se em [Mil], pag. 23.

Seja $f: M^{n} \rightarrow \mathbb{R}^{n+r}, r \geq 1$, uma imersão isométrica. De um modo geral, se $\xi$ é um vetor unitário fixo em $\mathbb{R}^{n+r}$, definimos a função altura de $f$ na direção $\xi$ por

$$
h_{\xi}(p):=<f(p), \xi>, \forall p \in M
$$

Se $\alpha$ é a segunda forma fundamental de $f$ e $\left(U, \varphi=\left(x_{1}, \ldots, x_{n}\right)\right)$ é um sistema de coordenadas em $p \in M$, temos

$$
\frac{\partial^{2} h_{\xi}}{\partial x_{i} \partial x_{j}}(p)=<\alpha\left(\frac{\partial}{\partial x_{i}}, \frac{\partial}{\partial x_{j}}\right)(p), \xi>
$$

De fato, seja $\left(V, \psi=\left(y_{1}, \ldots, y_{n+r}\right)\right)$ um sistema de coordenadas em $f(p)$ tal que

$$
\frac{\partial}{\partial y_{i}}(p)=Y_{i}, \quad \bar{\nabla}_{\frac{\partial}{\partial y_{i}}} \frac{\partial}{\partial y_{j}}(f(p))=0,
$$

onde $i, j=1, \ldots, n+r$, e $\left\{Y_{1}, \ldots, Y_{n+r}\right\}$ é base ortonormal de $T_{f(p)} \mathbb{R}^{n+r}$ tal que $Y_{i} \in$ $d f_{p}\left(T_{p} M\right), i=1, \ldots, n$ e $\bar{\nabla}$ é a conexão riemanniana de $\mathbb{R}^{n+r}$. Então,

$$
\alpha\left(\frac{\partial}{\partial x_{i}}, \frac{\partial}{\partial x_{j}}\right)(p)=\sum_{k=1}^{n+r} \frac{\partial^{2}\left(y_{k} \circ f\right)}{\partial x_{i} \partial x_{j}}(p) Y_{k} .
$$

Como

$$
\frac{\partial h_{\xi}}{\partial x_{j}}(p)=\frac{\partial\left(h_{\xi} \circ \varphi^{-1}\right)}{\partial x_{j}}(\varphi(p))
$$


concluimos que

$$
\frac{\partial^{2} h_{\xi}}{\partial x_{i} \partial x_{j}}(p)=\sum_{k=1}^{n+r} \frac{\partial^{2}\left(y_{k} \circ f\right)}{\partial x_{i} \partial x_{j}}(p)<Y_{k}, \xi>_{f(p)}=<\alpha\left(\frac{\partial}{\partial x_{i}}, \frac{\partial}{\partial x_{j}}\right)(p), \xi>.
$$

Seja $M^{n}$ variedade orientável e $f: M \rightarrow \mathbb{R}^{n+1}$ uma imersão isométrica com campo normal unitário $\xi$. A aplicação normal de Gauss $\phi: M^{n} \rightarrow S^{n} \subset \mathbb{R}^{n+1}$ associa a cada ponto $p \in$ $M$ o vetor $\phi(p)=\xi_{p} \in S^{n}$ que é obtido pela identificação $T_{f(p)} \mathbb{R}^{n+1} \equiv \mathbb{R}^{n+1}$. Como $d f_{p}\left(T_{p} M\right)$ e $T_{\phi(p)} S^{n}$ são paralelos, podemos olhar $d \phi_{p}: T_{p} M \rightarrow T_{\phi(p)} S^{n}$ como $d \phi_{p}: T_{p} M \rightarrow T_{p} M$, e isto feito, temos que

$$
d \phi_{p}=-A_{\xi_{p}} .
$$

Então, se $h_{\xi_{p}}(p)=<f(p), \xi_{p}>$ é a função altura na direção $\xi_{p}$, por ( 1.7$)$ temos que

$$
\frac{\partial^{2} h_{\xi_{p}}}{\partial x_{i} \partial x_{j}}(p)=<A_{\xi_{p}}\left(\frac{\partial}{\partial x_{i}}\right),\left(\frac{\partial}{\partial x_{j}}\right)>=<-d \phi_{p}\left(\frac{\partial}{\partial x_{i}}\right), d f_{p}\left(\frac{\partial}{\partial x_{j}}\right)>.
$$

\subsection{Colagem de alças em variedades diferenciáveis}

Aqui, chamaremos à bola aberta de raio $r$ em $\mathbb{R}^{n}$ de $r B^{n} \subset \mathbb{R}^{n}$ e faremos a identificação da coroa esférica $\left(\bar{B}^{n}-\frac{1}{3} B^{n}\right)$ com o produto cartesiano $S^{n-1} \times\left(\frac{1}{3}, 1\right)$, onde $\bar{B}^{n}$ denota o fecho de $B^{n}$.

Trabalharemos apenas no caso em que a variedade é orientável.

Iniciaremos a construção da colagem de alças numa variedade diferenciável $M^{n}$ tomando mergulhos $f_{1}, f_{2}: B^{n} \rightarrow M^{n}$ com as seguintes propriedades: $f_{1}$ preserva e $f_{2}$ inverte orientação e $f_{1}\left(B^{n}\right) \cap f_{2}\left(B^{n}\right)=\emptyset$. Denotaremos por $M_{f}$ a variedade diferenciável obtida a partir de $M-\left(f_{1}\left(\frac{1}{3} B^{n}\right) \cap f_{2}\left(\frac{1}{3} B^{n}\right)\right)$ pela identificação $f_{1}(t x)=f_{2}((1-t) x)$, para $t \in\left(\frac{1}{3}, \frac{2}{3}\right), \quad x \in S^{n-1}$, como é mostrado na figura 1.1. 


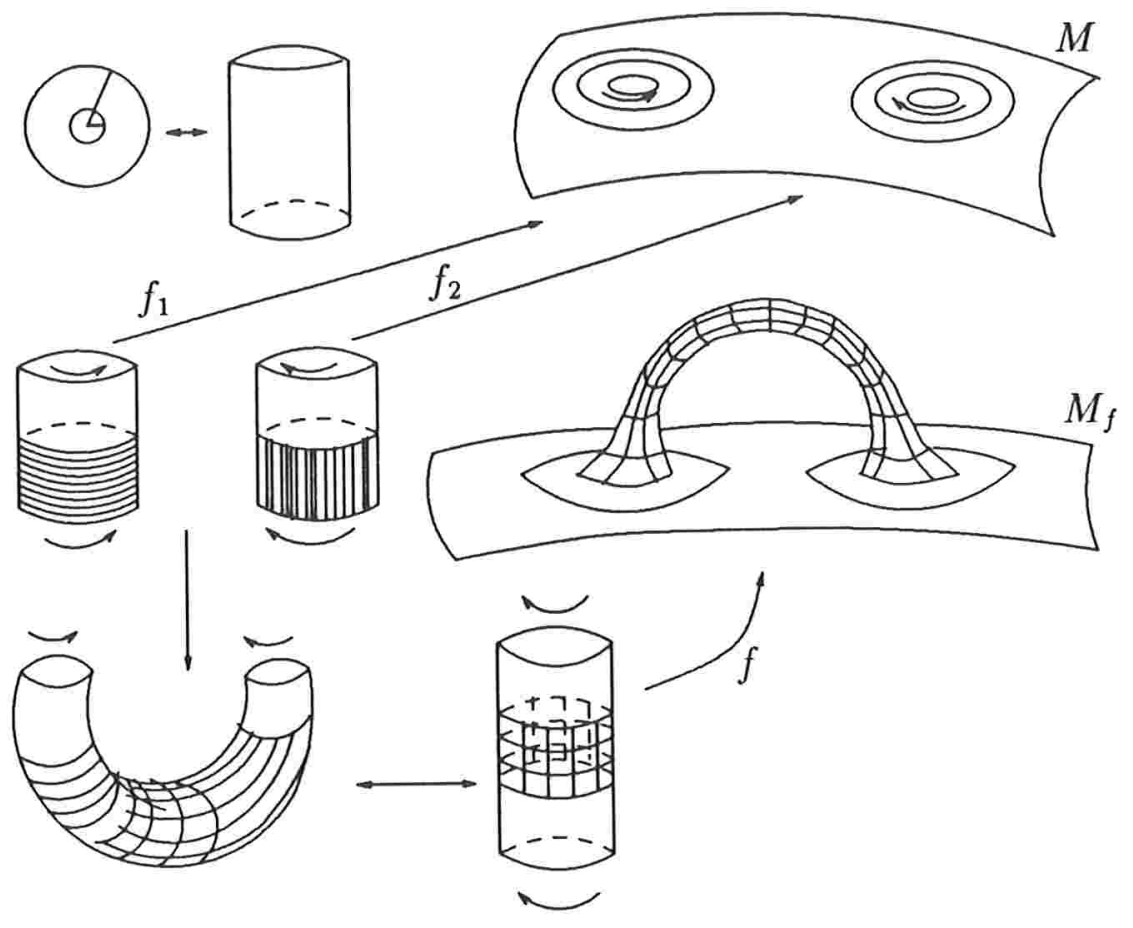

Figura 1.1: esquema de colagem de alça

Observamos que a variedade obtida é diferenciável e que, se tomarmos um outro par de mergulhos $g_{1}, g_{2}$ satisfazendo as propriedades citadas anteriormente, a variedade obtida é difeomorfa a $M_{f}$. Diremos que a variedade $M_{f}$ é obtida de $M$ a partir da colagem de uma alça.

Chamaremos $S_{b}^{n}$ à variedade obtida de $S^{n}$ após a colagem de $b$ alças. 


\section{Apêndice A}

\section{Uma demonstração do Teorema de Weyl}

No que se segue, daremos uma demonstração do Teorema 1.3.2, onde usaremos a teoria das subvariedades do espaço de Lorentz $\mathbb{L}^{n}$. Uma exposição recente deste material encontrase no capítulo 4 de [O'N]. Observamos aqui que o enunciado e a demonstração do teorema fundamental das subvariedades na sua versão lorentziana (que é o que usaremos a seguir) segue as mesmas linhas do caso euclidiano, cujo enunciado preciso é o Teorema 1.2.2. Os argumentos abaixo estão largamente baseados na seção 2 de [A-D].

\section{Demonstração do Teorema 1.3.2:}

$(\Longrightarrow) \operatorname{Seja}\left(M^{n},<,>\right)$ uma variedade conformemente plana segundo a Definição 1.3.1. Como (i) e (ii) são condições pontuais podemos supor que $M^{n}$ é ela toda conforme a um aberto do $\mathbb{R}^{n}$. Segue-se daí que existe uma aplicação conforme $F: M \rightarrow S^{n}$, onde estaremos olhando $S^{n}$ como o subconjunto $\bar{S}^{n}=\left\{x \in V^{n+1}:\left(x, e_{n+2}\right)=-1\right\}$, onde $e_{n+2}=(0, \ldots, 0,1) \in \mathbb{L}^{n+2}$ e $V^{n+1}$ é o cone de luz definido em (1.5). Resumindo, existem $\lambda: M \rightarrow \mathbb{R}$ função positiva e $F: M \rightarrow \bar{S}^{n} \subset V^{n+1}$ tais que

$$
(d F X, d F Y)=\lambda^{2}<X, Y>
$$


para todo $p \in M$ e $X, Y \in T_{p} M$. Como $(F, F)=0$, temos que

$$
\left(d F_{p} X(p), F(p)\right)=0 \quad \forall X \in T_{p} M .
$$

Tomando $\mu=\frac{1}{\lambda}$ e $f=\mu F$, temos que $f(M) \subset V^{n+1}$ e, pela observação acima,

$$
(d f X, d f Y)=<X, Y>
$$

Assim, construimos uma imersão isométrica $f: M \rightarrow V^{n+1} \subset \mathbb{L}^{n+2}$. Sejam $i: V^{n+1} \rightarrow \mathbb{L}^{n+2}$ a aplicação inclusão e $\delta: M \rightarrow \mathbb{L}^{n+2}$ dada por $\delta=i \circ f$. Pelas mesmas razões acima temos que

$$
\left(d \delta_{p} X(p), d \delta_{p} Y(p)\right)=0 \quad \forall p \in M, \quad \forall X, Y \in T_{p} M
$$

Então podemos pensar em $\delta$ como sendo ele mesmo um campo normal à imersão $\delta$. Sendo $\bar{\nabla}$ a conexão métrica de $\mathbb{L}^{n+2}$, temos que $\bar{\nabla}_{X} \delta=d \delta X \equiv X$, donde $\bar{\nabla}_{X}^{\perp} \delta=0$, isto é, $\delta$ é paralelo na conexão normal $\bar{\nabla}^{\perp}$ do fibrado normal $T M^{\perp}$ da imersão $\delta$ e, além disso, a sua segunda forma fundamental $A_{\delta}$ satisfaz

$$
A_{\delta}=-I d
$$

Para $p \in M$ (arbitrário) fixo, $\left(T_{p} M\right)^{\perp}$ é um espaço de Lorentz de dimensão 2 e $\delta(p)$ é um vetor nulo neste espaço, daí existem $\xi_{p}$ e $\eta_{p}$ em $\left(T_{p} M\right)^{\perp}$ tais que $\delta_{p}=\xi_{p}-\eta_{p},-\left\|\xi_{p}\right\|^{2}=\left\|\eta_{p}\right\|^{2}=1$. Então, $A_{\xi_{p}}-A_{\eta_{p}}=A_{\delta_{p}}=-I d$. A partir daí, verifica-se que o tensor de curvatura normal $R^{\perp}$ da conexão $\bar{\nabla}^{\perp}$ é identicamente nulo e isto implica que existem extensôes locais de $\xi_{p}$ e $\eta_{p}$ a campos normais paralelos $\xi$ e $\eta$, que podem ser tomados globalmente definidos, quando $M^{n}$ é simplesmente conexa.

Como $\xi-\eta$ e $\delta$ são campos nulos em $\mathcal{X}(M)^{\perp}$ e como cada $\left(T_{q} M\right)^{\perp}$ é um espaço de Lorentz bidimensional, concluimos que

$$
\delta=\xi-\eta
$$

em $M$ pois $\delta(p)=\xi_{p}-\eta_{p}$. Seja $q \in M$ qualquer e $\left\{e_{1}, \ldots, e_{n}\right\}$ uma base ortonormal de $T_{q} M$ que diagonaliza simultaneamente $A_{\xi}$ e $A_{\eta}$, isto é, $A_{\xi} e_{i}=\lambda_{i} e_{i}$ e $A_{\eta} e_{i}=\mu_{i} e_{i}$ para bem determinados números reais $\lambda_{i}$ e $\mu_{i}, i=1, \ldots, n$. Segue imediatamente de (A.1) e (A.2) que $\lambda_{i}-\mu_{i}=-1 \mathrm{e}$, com isto e com a equação de Gauss de $\delta$ obtemos

$$
k\left(e_{i}, e_{j}\right):=R\left(e_{i}, e_{j}, e_{j}, e_{i}\right)=1+\lambda_{i}+\lambda j .
$$


Além disso, substituição direta na definição de $L$ nos dá que

$$
L\left(e_{i}, e_{j}\right)=\frac{1+2 \lambda_{i}}{2} \delta_{i j}
$$

o que implica que

$$
L(X, Y)=\frac{<X, Y>}{2}+<A_{\xi} X, Y>
$$

Segue daí e de (A.2) que

$$
<A_{\xi} X, Y>=L(X, Y)-\frac{<X, Y>}{2},<A_{\eta} X, Y>=L(X, Y)+\frac{<X, Y>}{2}
$$

para todo $X, Y \in \mathcal{X}(M)$.

Usando (A.4), verificamos que as equações de Gauss e Codazzi implicam nas condições (mais que isso, são equivalentes a) (i) e (ii) do Teorema 1.3.2, respectivamente, o que finaliza a primeira implicação do mesmo.

( ) Reciprocamente, se são válidas (i) e (ii) podemos supor, sem perda de generalidade, que $M$ é simplesmente conexa e vamos mostrar, inicialmente, que existe uma imersão isométrica $g: M^{n} \rightarrow \mathbb{L}^{n+2}$ com $g(M)$ contida em algum cone de luz de $\mathbb{L}^{n+2}$. Para tanto, consideremos o fibrado vetorial trivial $T=M^{n} \times \mathbb{L}^{2}$ munido de uma conexão compatível $\nabla^{\prime}$ que torna paralelas as seç̧ões $\xi(q)=\left(q, e_{1}\right)$ e $\eta(q)=\left(q, e_{2}\right)$, onde $e_{1}=(1,0)$ e $e_{2}=(0,1)$.

Definimos agora endomorfismos $A_{\xi}$ e $A_{\eta}$ de $T M$ por

$$
<A_{\xi} X, Y>=L(X, Y)-\frac{<X, Y>}{2},<A_{\eta} X, Y>=L(X, Y)+\frac{<X, Y>}{2}
$$

e a forma bilinear simétrica $\beta: T M \times T M \rightarrow T$ por

$$
\beta(X, Y):=-<A_{\xi} X, Y>\xi+<A_{\eta} X, Y>\eta
$$

Cálculos diretos utilizando as definições acima mostram que (i) e (ii) implicam que $\nabla^{\prime}, \beta$ e os dados riemannianos de $M$ verificam as equações de Gauss e Codazzi de uma imersão isométrica $M^{n}$ em $\mathbb{L}^{n+2}$; a respectiva equação de Ricci decorre do fato de $A_{\xi}$ e $A_{\eta}$ poderem ser simultaneamente diagonalizados. Em vista da versão lorentziana do teorema fundamental das subvariedades (veja-se início deste apêndice), existe uma imersão isométrica $g: M^{n} \rightarrow \mathbb{L}^{n+2}$ com segunda forma fundamental $\beta$ e com conexão normal $\nabla^{\prime}$. Para mostrar que $g(M)$ está 
contida em algum cone de luz de $\mathbb{L}^{n+2}$, consideremos $h=g-(\xi-\eta)$. Então, para qualquer $X \in \mathcal{X}(M)$ temos

$$
X(h)=X(g)-X(\xi-\eta)=X-\left(\nabla_{X}^{\prime} \xi-A_{\xi} X-\nabla_{X}^{\prime} \eta+A_{\eta} X\right)=X+A_{\xi-\eta} X .
$$

Pela definição de $A_{\xi}$ e $A_{\eta}$ concluimos que $A_{\xi-\eta}=-I d$, logo

$$
X(h)=0 .
$$

Consequentemente, $h=$ cte, $g=h+(\xi-\eta),(g-h, g-h)=0$ e $g-h \neq 0$. Assim, $g(M)$ está contida num cone de luz de vértice $h$.

Observamos que temos, neste ponto, uma demonstração do Teorema 1.3.4.

Tomemos agora $f: M \rightarrow \mathbb{L}^{n+2}$ dada por $f=g-h$. Claramente $f$ é uma imersão isométrica e $(f, f)=(g-h, g-h)=0$, donde, a menos de se trocar $f$ por $-f$, temos que $f(M) \subset V^{n+1}$. Segue-se daí que $\lambda: M \rightarrow \mathbb{R}$ dada por $\lambda(p)=\left(f(p), e_{n+2}\right)$ é diferenciável e positiva em $M$. Definindo $F: M \rightarrow V^{n+1} \subset \mathbb{L}^{n+2}$ por $F=-\lambda^{-1} f$, observamos que $\left(F, e_{n+2}\right) \equiv-1$, ou seja $F(M) \subset \bar{S}^{n}$, e que $(F, F) \equiv 0$, donde $(d F X, F)=0$ e, consequentemente,

$$
(d F X, d F Y)=\frac{1}{\lambda^{2}}(d f X, d f Y)=\frac{1}{\lambda^{2}}\langle X, Y\rangle,
$$

para todo $X, Y \in \mathcal{X}(M)$. Obtivemos assim uma imersão conforme $F: M^{n} \rightarrow V^{n+1} \subset \mathbb{L}^{n+2}$ com $F(M) \subset \bar{S}^{n} \equiv S^{n}$, donde $M$ é conformemente plana. 


\section{Capítulo 2}

\section{Hipersuperfícies conformemente planas}

Neste capítulo vamos estudar hipersuperfícies conformemente plana compactas $f: M^{n} \rightarrow$ $\tilde{M}^{n+1}(c), n \geq 4$, onde $\tilde{M}^{n+1}(c)$ é uma variedade riemanniana completa, simplesmente conexa, de curvatura seccional constante igual a $c$. Como $\tilde{M}^{n+1}(c)$ é conformemente difeomorfa, a menos de um ponto (no caso $c>0$ ), a $\mathbb{R}^{n+1}$ e $f\left(M^{n}\right)$ é compacta, assumiremos nas demonstrações (e portanto no enunciado de alguns lemas), que $f$ é uma hipersuperfície compacta de $\mathbb{R}^{n+1}$.

\subsection{Hipersuperfícies conformemente planas compactas}

O principal resultado desta seção é o teorema 2.1.2, um teorema sobre a estrutura das componentes conexas dos conjuntos de pontos umbílicos e não umbílicos de $M^{n}$. Chamaremos o conjunto dos pontos umbílicos de $M^{n}$ de $U$. Utilizaremos em vários momentos o teorema a seguir, cuja demonstração pode ser encontrada em [D],pag. 118, Teorema 7.11.

Teorema 2.1.1 Seja $f: M^{n} \rightarrow \tilde{M}^{n+1}(c), n \geq 4$, uma imersão isométrica. Então $M^{n} e^{\prime}$ conformemente plana se, e somente se, $f$ tem uma curvatura principal de multiplicidade maior 
ou igual $a(n-1)$.

O resultado principal desta seção é o

Teorema 2.1.2 Seja $f: M^{n} \rightarrow \tilde{M}^{n+1}(c)$ uma hipersuperfície conformemente plana imersa, compacta e conexa, $n \geq 4$. Vamos assumir $U$ não vazio. Então

(i) cada componente conexa $D$ do conjunto $M-U$ admite uma folheação de codimensão 1 por $(n-1)$-esferas; em particular $D$ é difeomorfo a $S^{n-1} \times(a, b)$;

(ii) cada componente conexa de $U$ é um ponto, uma (n-1)-esfera ou um conjunto $n$-dimensional umbílico limitado por pontos ou pela união de $(n-1)$-esferas, tal que a união de quaisquer duas delas tenha, no máximo, um ponto em comum.

A demonstração do item (i) do Teorema 2.1.2 está basicamente no seguinte Lema

Lema 2.1.3 Seja $M^{n}$ uma variedade conformemente plana compacta, $n \geq 4$, e seja $f: M^{n} \rightarrow$ $\mathbb{R}^{n+1}$ uma imersão isométrica. Então o conjunto $M-U$ tem uma folheação de codimensão 1 tal que a imagem $f(\Sigma)$ de cada folha $\Sigma$ é uma $(n-1)$-esfera. Em particular, cada componente conexa aberta de $M-U$ é difeomorfa a $S^{n-1} \times(a, b)$.

Demonstração: Como estamos em codimensão 1, escolhemos uma orientação local $N$ numa vizinhança apropriada de cada ponto $p \in M-U$.

Sejam $\lambda$ e $\mu$ os autovalores de $A_{N}$, e $D_{\lambda}$ e $D_{\mu}$ as respectivas distribuições definidas tomandose, para cada ponto $p \in M-U$, o auto-espaço associado ao respectivo autovalor. Estas distribuições independem da orientação local, logo estão definidas em $M-U$. Além disso, por um resultado bastante conhecido ([Ry] pag. 372), e pela forma como foram definidas, as distribuições são diferenciáveis, involutivas e, como $\operatorname{dim} D_{\lambda}=n-1>1$, o autovalor $\lambda$ é constante ao longo de cada folha $\Sigma_{\lambda}$ da distribuição.

Isto indica que podemos tomar coordenadas locais $\left(u_{1}, u_{2}, \ldots, u_{n-1}, t\right)$ tais que os campos coordenados $V_{i}=\frac{\partial}{\partial u_{i}}$ e $T=\frac{\partial}{\partial t}$ satisfazem

$$
A_{N}\left(V_{i}\right)=\lambda V_{i}=-\bar{\nabla}_{V_{i}} N, \quad i=1, \ldots, n-1,
$$




$$
A_{N}(T)=\mu T=-\bar{\nabla}_{T} N
$$

onde $\bar{\nabla}$ denota a conexão euclidiana de $\mathbb{R}^{n+1}$. Como $\mathbb{R}^{n+1}$ é plano, temos que $R\left(V_{i}, T\right) N=0$. Como T e $V_{i}$ são campos coordenados em $\mathrm{M},\left[T, V_{i}\right] \equiv 0$, o que nos dá

$$
\begin{gathered}
\bar{\nabla}_{V_{i}} T=\bar{\nabla}_{T} V_{i}, \\
\bar{\nabla}_{V_{i}} \bar{\nabla}_{T} N-\bar{\nabla}_{T} \bar{\nabla}_{V_{i}} N=0 .
\end{gathered}
$$

Usando (2.1) e (2.2), concluímos de (2.4) que

$$
\lambda^{\prime} V_{i}+(\lambda-\mu) \bar{\nabla}_{T} V_{i}-V_{i}(\mu) T=0,
$$

onde $\lambda^{\prime}=\frac{d \lambda}{d t}$. Como $\lambda \neq \mu$ em $M-U$, temos

$$
\bar{\nabla}_{T} V_{i}=-\frac{\lambda^{\prime}}{\lambda-\mu} V_{i}+\frac{V_{i}(\mu)}{\lambda-\mu} T=\bar{\nabla}_{V_{i}} T
$$

Consideremos agora a folha $\Sigma_{\lambda} \subset M$ e seja $i: \Sigma_{\lambda} \rightarrow M$ a aplicação inclusão. Então, se $\|T\|=\alpha^{-1}$ e $\tilde{A}_{N}$ e $\tilde{A}_{\alpha T}$ são os operadores de Weingarten de $f \circ i: \Sigma_{\lambda} \rightarrow \mathbb{R}^{n+1}$, obtemos respectivamente

$$
\begin{gathered}
\tilde{A}_{N}\left(V_{i}\right)=\left(-\bar{\nabla}_{V_{i}} N\right)^{T_{\Sigma_{\lambda}}}=-\bar{\nabla}_{V_{i}} N=\lambda V_{i} \\
\tilde{A}_{\alpha T}\left(V_{i}\right)=\left(-\bar{\nabla}_{V_{i}} \alpha T\right)^{T_{\Sigma_{\lambda}}}=\left(-V_{i}(\alpha) T-\alpha \bar{\nabla}_{V_{i}} T\right)^{T_{\Sigma_{\lambda}}}=\alpha \frac{\lambda^{\prime}}{\lambda-\mu} V_{i}
\end{gathered}
$$

onde ( $)^{T_{\Sigma_{\lambda}}}$ significa a projeção ortogonal no espaço tangente a $\Sigma_{\lambda}$. Decorre de (2.6) e (2.7) que $\Sigma_{\lambda}$ é umbílica relativamente à imersão $f$ o $i$. Podemos tomar uma base ortonormal do plano $\{T, N\}$ formada por vetores $\xi$ e $\xi^{\perp}$ tal que o operador $\tilde{A}$ satisfaça $\tilde{A}_{\xi}\left(V_{i}\right)=\beta V_{i}$ e $\tilde{A}_{\xi^{\perp}}\left(V_{i}\right)=0$, para $i=1, \ldots, n-1$, sendo $\beta^{2}=\left(\frac{\lambda^{\prime}}{\lambda-\mu} \alpha\right)^{2}+\lambda^{2}$ a curvatura seccional de $\Sigma_{\lambda}$. Veja figura 2.1.

De fato, se $\beta \neq 0$ tomamos

$$
\begin{aligned}
\xi & =\left(\alpha \frac{\lambda^{\prime}}{\lambda-\mu} \alpha T+\lambda N\right) \beta^{-1} \\
\xi^{\perp} & =\left(\lambda \alpha T-\alpha \frac{\lambda^{\prime}}{\lambda-\mu} N\right) \beta^{-1}
\end{aligned}
$$

e temos

$$
<\xi, \xi^{\perp}>=\left(\alpha \frac{\lambda^{\prime}}{\lambda-\mu} \lambda \alpha^{2}(\|T\|)^{2}-\lambda \alpha \frac{\lambda^{\prime}}{\lambda-\mu}\right) \beta^{-2}=0
$$




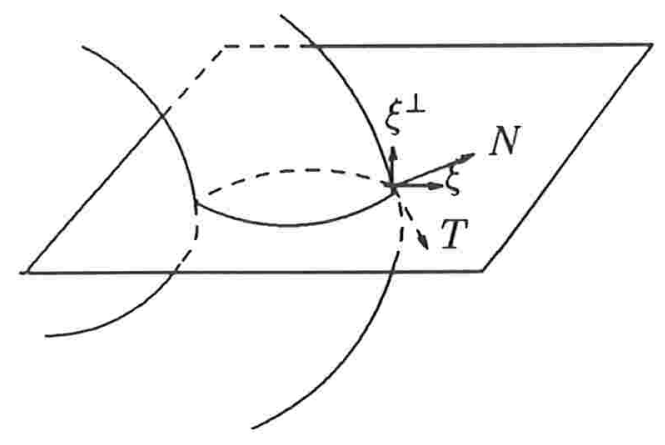

Figura 2.1: existência de $\xi$ e $\xi^{\perp}$

e

$$
\tilde{A}_{\xi}\left(V_{i}\right)=\left(-\bar{\nabla}_{V_{i}} \xi\right)^{T_{\Sigma_{\lambda}}}=\left(-\bar{\nabla}_{V_{i}}\left(\frac{\lambda^{\prime}}{\lambda-\mu} \alpha T+\lambda N\right) \beta^{-1}\right)^{T_{\Sigma_{\lambda}}}=\beta^{-1}\left(\alpha^{2}\left(\frac{\lambda^{\prime}}{\lambda-\mu}\right)^{2}+\lambda^{2}\right) V_{i}=\beta V_{i}
$$

Além disso,

$$
\tilde{A}_{\xi^{\perp}}\left(V_{i}\right)=\left(-\bar{\nabla}_{V_{i}} \xi^{\perp}\right)^{T_{\Sigma_{\lambda}}}=\left(-\bar{\nabla}_{V_{i}}\left(\lambda \alpha T-\frac{\lambda^{\prime}}{\lambda-\mu} \alpha N\right) \beta^{-1}\right)^{T_{\Sigma_{\lambda}}}=0
$$

Se $\beta=0, \tilde{A} \equiv 0$ para qualquer vetor normal à folha $\Sigma_{\lambda}$ (veja (2.6) e (2.7) ).

Até aqui, vimos que em cada ponto $p \in M$ passa uma folha $\Sigma_{\lambda}$ tal que $f\left(\Sigma_{\lambda}\right)$ está contida num $(n-1)$-plano (se $\beta=0$ ) ou numa $(n-1)$-esfera (se $\beta \neq 0$ ). Resta-nos mostrar que $f\left(\Sigma_{\lambda}\right)$ é uma $(n-1)$-esfera. Para tanto utilizaremos o seguinte teorema de Reckziegel, veja pag. 8 de $[R e]$.

"Se G é um subconjunto de $M$ no qual a função $p \longmapsto \operatorname{dim} D_{\lambda}(p)$ (auto-espaço associado ao auto-valor $\lambda)$ é minimal, $\lambda$ é covariante constante ao longo de $D_{\lambda}(p)$ e $M$ é completa, então, todas as folhas de $D_{\lambda}$ também são espaços completos.”

Tomando $G=M-U$ e observando que $\mathrm{M}$ é completa (M é compacta), $\operatorname{dim} D_{\lambda}(p)=n-1$ é minimal para todo $p$ em $M-U$ e $\lambda$ é constante ao longo de cada folha, (constante ao longo de $\left.D_{\lambda}\right)$, concluimos que $\Sigma_{\lambda}$ é completa. Então $f\left(\Sigma_{\lambda}\right)$ é um $(n-1)$-plano ou uma $(n-1)$-esfera. Como $f\left(\Sigma_{\lambda}\right) \subset f(M)$ e $M$ é compacta, $f\left(\Sigma_{\lambda}\right)$ é uma $(n-1)$-esfera, portanto $\beta \neq 0$. 
Observemos que $\Sigma_{\lambda}$ é completa e de curvatura seccional constante positiva, e $f\left(\Sigma_{\lambda}\right)$ é uma $(n-1)$-esfera. Como $f$ é imersão isométrica, $\Sigma_{\lambda}$ é uma $(n-1)$-esfera.

É razoavelmente claro neste ponto que $D$ é difeomorfo a $S^{n-1} \times(a, b)$, porém um argumento um pouco mais preciso demonstrando esse fato será apresentado em 2.2.2.

Corolário 2.1.4 Se $M$ é uma hipersuperfície conformemente plana compacta sem pontos umbiticos então, se $M$ é orientável, $M$ é homeomorfa a $S^{n-1} \times S^{1}$ e, caso contrário, $M$ é homeomorfa a uma "garrafa de Klein generalizada".

Demonstração: Veja comentários em 2.2 .2

Lema 2.1.5 Seja $U$ não vazio e $D$ uma componente conexa de $M-U$. Então sua fronteira $\partial D$ tem no máximo duas componentes conexas e cada componente é um ponto, uma $(n-1)$-esfera ou a união de duas $(n-1)$-esferas com um ponto em comum.

Demonstração: Seja $p \in \partial D$ e seja $\left\{p_{j}\right\}$ uma sequência de pontos com $p_{j} \in D$ e $p_{j} \rightarrow p$. Então temos pelo lema anterior que, para cada $j \in I N$ existe uma $(n-1)$-esfera $\Sigma_{j} \subset M$ passando por $p_{j}$. Podemos escolher a sequência $\left\{p_{j}\right\}$ de tal forma que $\Sigma_{j} \neq \Sigma_{m}$ se $m \neq j$. Isto é possível pois se existisse $\Sigma_{j}$ tal que $\Sigma_{j}=\Sigma_{i}, i \neq j$ e $i \in I \subsetneq I N$ com $\sharp I=\infty$, a sequência $\left\{p_{i}\right\}$ seria tal que $p_{i} \in \Sigma_{j}, \forall i \in I$. Como $\Sigma_{j}$ é completa e está contida em $M$ (que é compacta), então $\Sigma_{j}$ é compacta. Então existe uma subsequência $\left\{p_{i_{k}}\right\}$ de $\left\{p_{i}\right\}$ que é convergente. Como $p_{j} \rightarrow p$ então $p_{i_{k}} \rightarrow p$ e teríamos $p \in \Sigma_{j} \subset D$ o que seria uma contradição. Podemos ainda assumir que, para cada $j, p_{j} \in V$ onde $V \subset M$ é uma vizinhança de $p$ tal que $f_{\left.\right|_{V}}$ é um mergulho.

Sejam agora $T_{j}=T_{f\left(p_{j}\right)}\left(f\left(\Sigma_{j}\right)\right), r_{j}=$ raio de $f\left(\Sigma_{j}\right)$ e $\xi_{j}$ vetor unitário normal a $f\left(\Sigma_{j}\right)$ em $f\left(p_{j}\right)$ e contido no hiperplano afim que contém $f\left(\Sigma_{j}\right)$, aqui chamado de $\left(\mathbb{R}^{n}\right)_{j}$. Definimos assim, sequências $\left\{T_{j}\right\},\left\{r_{j}\right\},\left\{\xi_{j}\right\},\left\{\left(\mathbb{R}^{n}\right)_{j}\right\}$ e $\left\{\Sigma_{j}\right\}$. Veja figura 2.2.

Queremos concluir que $T_{j} \rightarrow T, r_{j} \rightarrow r, \xi_{j} \rightarrow \xi, \Sigma_{j} \rightarrow \Sigma$ e $\left(\mathbb{R}^{n}\right)_{j} \rightarrow\left(\mathbb{R}^{n}\right)_{\Sigma}$, onde $\Sigma$ é uma $(n-1)$-esfera de raio $r$ contida em $\left(\mathbb{R}^{n}\right)_{\Sigma}$ e tal que o vetor $T$ lhe é tangente em $f(p)$. 


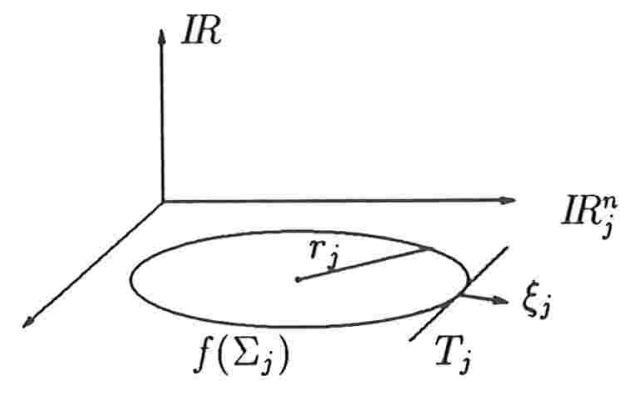

Figura 2.2: definição das sequências

Para concluirmos o que foi dito, iniciamos fazendo a identificação de cada $p_{j}$ com a origem da $n$-esfera unitária $S^{n}$, interceptando $\left(\mathbb{R}^{n}\right)_{j} \operatorname{com} S^{n}$, veja figura 2.3. Então, a sequência $\left\{\xi_{j}\right\}$ está agora em $S^{n}$ que é compacta, logo, existe subsequência $\left\{\xi_{j_{k^{\prime}}}\right\}$ tal que $\xi_{j_{k^{\prime}}} \rightarrow \xi$. Tomemos agora a sequência $\left\{N_{j_{k^{\prime}}}\right\}$ de vetores normais unitários a cada $\left(\mathbb{R}^{n}\right)_{j_{k^{\prime}}}$. Analogamente à sequência anterior, esta também é limitada, logo existe $\left\{N_{j_{k^{\prime \prime}}}\right\}$ tal que $N_{j_{k^{\prime \prime}}} \rightarrow N \in S^{n}$. Então, este vetor unitário é normal a um único hiperplano $\left(\mathbb{R}^{n}\right)_{\Sigma}$. É claro que $\left(\mathbb{R}^{n}\right)_{j_{k^{\prime \prime}}} \rightarrow\left(\mathbb{R}^{n}\right)_{\Sigma}$. Além disso, ainda temos $\xi_{j_{k^{\prime \prime}}} \rightarrow \xi$, onde $\xi \perp N$ portanto $\xi / /\left(\mathbb{R}^{n}\right)_{\Sigma}$. Consideremos $T$ o (único) hiperplano de $\left(\mathbb{R}^{n}\right)_{\Sigma}$ tal que $\xi \perp T$. Da mesma forma que acima, $T_{j_{k^{\prime \prime}}} \rightarrow T$ pois $\xi_{j_{k^{\prime \prime}}} \rightarrow \xi$. A sequência dada por $\left\{r_{j_{k^{\prime \prime}}}\right\}$ é limitada devido à compacidade de $M$, logo existe subsequência $\left\{r_{j_{k}}\right\}$ tal que $r_{j_{k}} \rightarrow r$. Daí, $r_{j_{k}} \rightarrow r, \xi_{j_{k}} \rightarrow \xi, T_{j_{k}} \rightarrow T$ e $\left(\mathbb{R}^{n}\right)_{j_{k}} \rightarrow\left(\mathbb{R}^{n}\right)_{\Sigma}$, o que nos dá $\Sigma_{j_{k}} \rightarrow \Sigma$, onde $\Sigma$ é uma $(n-1)$ - esfera que satisfaz os requisitos impostos por nós inicialmente.
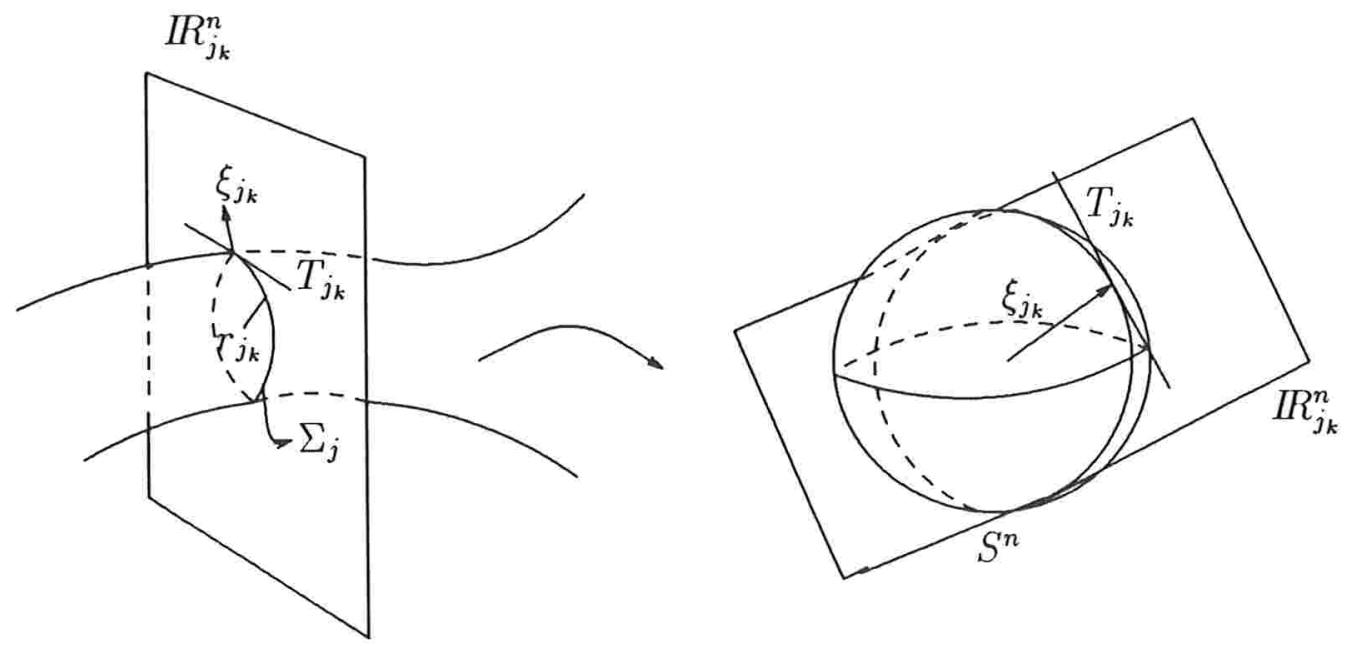

Figura 2.3: identificação em $S^{n}$ 
Como $U \subset M$ é fechado, $\partial U \subset U$. Além disso, $\partial U=\partial(M-U)$. Da conexidade de $D$ vem que $\partial D \subset \partial(M-U)=\partial U$, ou seja, $p \in U$. Ora, $p \in U$ e $p \in \Sigma$, logo $\Sigma \subset U$. De fato, suponhamos que exista $q \in \Sigma$ tal que $q \notin U$. Então $q$ não é umbílico e, pelo Lema 2.1.3 temos que uma $(n-1)$-esfera $\Sigma_{q}$ da folheação que passa por $q$. Então $\Sigma_{q} \equiv \Sigma$ e $\Sigma \subset M-U$, absurdo!!

Como $\partial D \subset U$, usando argumento análogo temos que $\Sigma \subset \partial D$. Notamos ainda que, se $r=\lim _{j \rightarrow \infty} r_{j}=0$, então $\Sigma$ se reduz a um ponto.

Observemos que $D$ não é compacto pois $U \neq \emptyset$. Pelo Lema 2.1.3 temos que $D$ é homeomorfo a $S^{(n-1)} \times(-1,1)$ (veja novamente 2.2 .2 ), e o homeomorfismo leva cada fatia $S^{(n-1)} \times\{t\}, t \in$ $(-1,1)$ sobre uma folha $\Sigma_{t}$ da folheação de $D$. Pelo que acabamos de ver, $\partial D$ é a união de $(n-1)$-esferas (possivelmente degeneradas).

Afirmação: Quando $t \rightarrow 1$, temos $\Sigma_{t}$ convergindo para uma única $(n-1)$-esfera (talvez degenerada) $\Sigma_{1}$.

De fato, suponhamos que existam sequências $\Sigma_{t}$ e $\Sigma_{t_{m}}^{\prime}$ convergindo para $\Sigma_{1}$ e $\Sigma_{1}^{\prime}$ respectivamente, $\operatorname{com} \Sigma_{1} \neq \Sigma_{1}^{\prime}$. Sejam $p \in \Sigma_{1}, p \notin \Sigma_{1}^{\prime}$ e $V$ uma vizinhança conexa de $p$. Então, se $V$ é suficientemente pequena, $V$ intercepta $\Sigma_{t_{j}}$ e não intercepta $\Sigma_{t_{m}}^{\prime}$ para $m$ e $j$ suficientemente grande. Consideremos a imagem inversa de $\left(V \cap\left(\bigcup_{t, t_{j}, 0} \Sigma_{t_{j}}\right)\right)$ pelo homeornorfismo descrito acima. Este conjunto é conexo e é dado por $\left(S^{(n-1)} \cap \tilde{V}\right) \times(\alpha, 1)$, onde $\tilde{V}$ é aberto em $\mathbb{R}^{n}$ e $\alpha<1$. Tomemos $\Sigma_{t_{m}}^{\prime}$ tal que $\Sigma_{t_{m}}^{\prime} \cap V=\emptyset$ e $m$ suficientemente grande tal que $t_{m}>\alpha$. Então, $t_{m}$ não pertence à imagem inversa de $V \cap\left(\bigcup_{j>\jmath_{0}} \Sigma_{t_{j}}\right)$, o que a desconecta, contradição. De modo análogo concluímos que, se $t \rightarrow-1$ temos $\Sigma_{t} \rightarrow \Sigma_{-1}$ e $\Sigma_{-1}$ é única. Segue daí que $\partial D=\Sigma_{1} \cup \Sigma_{-1}$.

Se $\Sigma_{1}$ se reduz a um ponto $\left(\Sigma_{1}=q\right)$, então este ponto é uma componente conexa isolada de $\partial D$. Isso decorre do fato de que, se $V$ é uma vizinhança conexa de $q=\Sigma_{1}$ suficientemente pequena, então, para $t$ suficientemente próximo de 1 temos $\Sigma_{t} \subset V$. Mas, $\Sigma_{t} \subset D \operatorname{logo}$, se tomarmos $p \in \partial D$ não nos será possível encontrar um caminho contínuo $\gamma: I \rightarrow \partial D$ ligando $p$ a $q=\Sigma_{1}$, pois $\gamma(I) \cap \Sigma_{t} \neq \emptyset$.

Concluimos então que $q=\Sigma_{1}$ é uma componente conexa isolada de $\partial D$ e $\partial D=\Sigma_{1} \cup \Sigma_{-1}$. Analogamente, se $\Sigma_{-1}$ também se reduzir a um ponto, ainda assim teremos $\Sigma_{1} \cap \Sigma_{-1}=\emptyset$. 
Vamos assumir agora que $\Sigma_{1}$ e $\Sigma_{-1}$ não são degeneradas. Mostraremos que, neste caso, temos $\Sigma_{1}$ e $\Sigma_{-1}$ tangentes ou disjuntas.

Suponhamos que ocorra o contrário, ou seja, que $\Sigma_{1}$ e $\Sigma_{-1}$ se interceptam transversalmente. Como o estado de transversalidade não se modifica por pequenas perturbações, tomemos $\Sigma_{t} \mathrm{e}$ $\Sigma_{-t}$ para um valor de $t$ próximo de 1 . Neste caso, $\Sigma_{t}$ e $\Sigma_{-t}$ ainda se interceptam transversalmente e $\Sigma_{t}$ e $\Sigma_{-t}$ pertencem à folheação de $D$, o que é um absurdo! Então, $\Sigma_{1} \cap \Sigma_{-1}=\{p\}$ ou $\Sigma_{1} \cap \Sigma_{-1}=\emptyset$ e, neste caso, $\Sigma_{1}$ e $\Sigma_{-1}$ são as componentes conexas de $\partial D$, o que completa a demonstração.

A demonstração do item (ii) do teorema 2.1.2 está no seguinte corolário

Corolário 2.1.6 Seja $M$ uma hipersuperfície compacta, conformemente plana e $U$ o conjunto de pontos umbilicos de $M$. Então, cada componente conexa de $U$ é um ponto, uma $(n-1)$ esfera ou um conjunto n-dimensional limitado por pontos ou pela união de $(n-1)$-esferas, tal que duas $(n-1)$-esferas tenham no máximo um ponto em comum.

Demonstração: Se interior de $U$ é vazio, $U=\partial U=\partial(M-U)$. Pelo Lema 2.1.5, cada componente conexa de $U$ é um ponto, uma $(n-1)$-esfera ou a união de duas $(n-1)$-esferas com um ponto em comum. Mostraremos que a última possibilidade não pode ocorrer.

Suponhamos que $C=\Sigma \cup \Sigma^{\prime}$ é uma componente conexa de $U$ com $\Sigma \cap \Sigma^{\prime}=\{q\}$, e sejam $\varepsilon>0$ e $V_{\varepsilon}=\{p \in M-U: d(p, \Sigma)<\varepsilon\}$, onde $d$ é a distância intrínseca da variedade $M$. Seja $\gamma:[-\delta, \delta] \rightarrow M$, diferenciável, parametrizada pelo comprimento de arco e tal que $\gamma(0)=q \in \Sigma, \gamma^{\prime}(0) \perp T_{q} \Sigma, \gamma([-\delta, \delta]) \subset V_{\varepsilon}$ e $\gamma([-\delta, \delta]) \cap \Sigma=\{q\}$. Consideremos as $(n-1)$ esferas da folheação que passam por $\gamma(\delta)$ e $\gamma(-\delta), \Sigma_{\gamma(\delta)}$ e $\Sigma_{\gamma(-\delta)}$ respectivamente. Então, como $\Sigma^{\prime} \cap V_{\varepsilon} \neq \emptyset, \Sigma_{\gamma(\delta)} \cap \Sigma^{\prime} \neq \emptyset$ ou $\Sigma_{\gamma(-\delta)} \cap \Sigma^{\prime} \neq \emptyset$, temos uma contradição pois $\Sigma^{\prime} \subset U$ e as outras duas $(n-1)$-esferas estão em $M-U$. Veja figura 2.4 .

Se interior de $U$ é não vazio, como $U \subset M$ então $U$ é um conjunto $n$-dimensional. Novamente temos $\partial U=\partial(M-U)$, logo, se $A$ é uma componente conexa de $U$, vale $\partial A \subset \partial U$ e então $\partial A$ é composta pela união de $(n-1)$-esferas com, no máximo, um ponto em comum e por pontos. Estes pontos são dados pelo limite de sequências de $(n-1)$-esferas com um ponto 


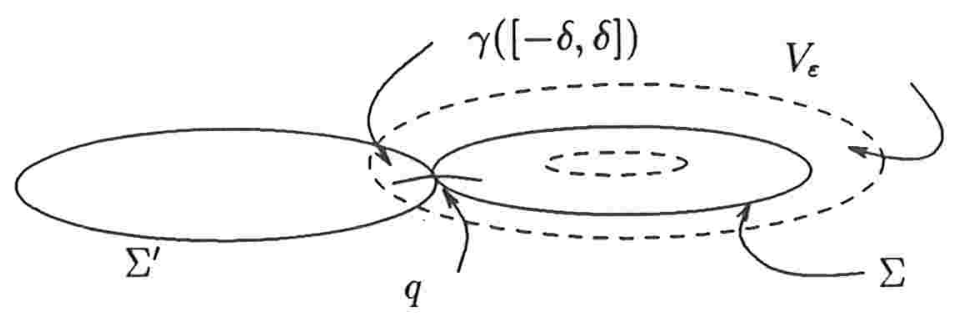

Figura 2.4:

em comum e raios que convergem para zero.

Para finalizar esta seção, observamos que os Lemas 2.1.3 e 2.1.5 e Corolário 2.1.6 nos dão o Teorema 2.1.2 no caso $c=0$. Os outros casos seguem-se das observações feitas no início do capítulo.

\subsection{Hipersuperfícies conformemente planas sem pon- tos umbílicos}

Nesta seção damos uma caracterização local das hipersuperfícies conformemente planas sem pontos umbílicos, introduzimos o conceito de vizinhança saturada e caracterizamos os pontos singulares destas mesmas hipersuperfícies, resultados que utilizaremos nas seções seguintes.

Seja $f: M^{n} \rightarrow \mathbb{R}^{n+1}, n \geq 4$, uma hipersuperfície conformemente plana sem pontos umbílicos e seja $p \in M$. Já vimos que existe numa vizinhança $W$ de $p$, um sistema de coordenadas $\left(u_{1}, \ldots, u_{n-1}, t\right)$ adaptado às distribuições $D_{\lambda}$ e $D_{\mu}$ e uma orientação local $N$. Sejam $V_{i}=\frac{\partial}{\partial u_{i}}, T=\frac{\partial}{\partial t}$ os campos coordenados em $W$, e $N$ uma orientação local tal que $<A_{N} V_{i}, V_{i}>=\lambda\left\|V_{i}\right\|^{2} \leq 0$.

Suponhamos $\lambda \neq 0$. Tomemos a aplicação $c(u, t)=f+\lambda^{-1} N$ e observemos que $\frac{\partial c}{\partial u_{i}}=0$ 
pois

$$
\frac{\partial f}{\partial u_{i}}+\frac{\partial}{\partial u_{i}}\left(\lambda^{-1} N\right)=\frac{\partial f}{\partial u_{i}}+\lambda^{-1} \frac{\partial N}{\partial u_{i}}=V_{i}-\lambda^{-1} A_{N}\left(V_{i}\right)=0,
$$

para $i=1,2, \ldots, n-1$. Isso implica que $c=c(t)$. Como $\lambda=\lambda(t)$, nós obtemos uma curva $c(t)$ e uma família a um parâmetro de $n$-esferas $\Sigma_{t}^{n}$, dadas por $\|y-c(t)\|^{2}=\lambda^{-2}$ tal que, para cada $t$, a esfera $\Sigma_{t}^{n}$ é tangente à hipersuperfície $f(M)$ pois $\|f-c(t)\|^{2}=\lambda^{-2}$. Como $f_{\mid W}$ satisfaz $\|f-c(t)\|^{2}=\lambda^{-2}$ e $\left\langle f-c, c^{\prime}\right\rangle=\lambda^{-3}=-\lambda^{-1}\left(\lambda^{-1}\right)^{\prime}, f$ é localmente a envolvente de uma família de esferas a um parâmetro de centros $c(t)$ e raios $-\lambda^{-1}(t)$.

Notemos que, se $\lambda=0$ a família de $n$-esferas torna-se um hiperplano tangente, o que, do ponto de vista da geometria conforme, é indistinguível de uma $n$-esfera pois, a menos de um ponto, ela é conformemente difeomorfa a um hiperplano $\mathbb{R}^{n}$. Logo podemos dizer que uma hipersuperfície conformemente plana sem pontos umbílicos é localmente a envolvente de uma família a um parâmetro de hipersuperfícies umbílicas. Podemos assumir, sem perda de generalidade, que $\lambda \neq 0$ pois, se num ponto $p \in M$ tem-se $\lambda=0$, podemos considerar $H$ o hiperplano tangente a $f(M)$ em $f(p)$, escolher $q \in \mathbb{R}^{n+1}$ tal que $q \notin H$ e $q \notin f(M)$ e, fazendo uma inversão $\tau$ em $\mathbb{R}^{n+1}$ com centro em $q$, obtemos que $\tau(H)$ é uma esfera, logo o valor de $\lambda$ em $p$ na hipersuperfície transformada $\tau \circ f: M^{n} \rightarrow \mathbb{R}^{n+1}$ é diferente de zero.

\subsubsection{Como construir uma hipersuperfície conformemente plana sem pontos umbílicos.}

Isso pode ser feito pela construção que veremos a seguir, a qual descreve uma envolvente de uma família a um parâmetro de $n$-esferas, $(\lambda \neq 0)$. Sejam $c:(a, b) \subseteq \mathbb{R} \rightarrow \mathbb{R}^{n+1}$ uma curva imersa e $r:(a, b) \subseteq \mathbb{R} \rightarrow \mathbb{R}$ uma função positiva real diferenciável que satisfaz $\left|r^{\prime}(t)\right|<\left\|c^{\prime}(t)\right\| \quad \forall t \in(a, b)$. Chamemos

$$
S=\frac{r r^{\prime}}{\left\|c^{\prime}\right\|^{2}}
$$

$\mathrm{e}$

$$
R=r\left[1-\left(\frac{r^{\prime}}{\left\|c^{\prime}\right\|}\right)^{2}\right]^{\frac{1}{2}}
$$

Para cada $t$ consideremos o hiperplano afim $\mathbb{R}_{t}^{n}$, de $\mathbb{R}^{n+1}$, que é ortogonal a $c^{\prime}(t)$ e passa por

$$
\gamma(t)=c(t)-S(t) c^{\prime}(t) .
$$


Ainda neste hiperplano, consideremos uma $(n-1)$-esfera $\sum_{t}^{n-1}$ com raio $R(t)$ e centro $\gamma(t)$. Então o conjunto descrito por $\Sigma_{t}^{n-1}$, quando $t$ percorre $(a, b)$, é a imagem da aplicação $y: S^{n-1} \times(a, b) \rightarrow \mathbb{R}^{n+1}$ dada por

$$
y(q, t)=c(t)-S c^{\prime}(t)+R \phi(q, t)=\gamma(t)+R \phi(q, t), \quad t \in(a, b), \quad q \in S^{n-1},
$$

onde $S^{n-1}$ é uma $(n-1)$-esfera unitária fixada num $n$-espaço euclidiano e $\phi: S^{n-1} \times(a, b) \rightarrow$ $\mathbb{R}^{n+1}$ é uma aplicação diferenciável que, para $t$ fixado satisfaz

(i) $\phi_{t}: S^{n-1} \rightarrow \mathbb{R}^{n+1}$ é uma imersão;

(ii) $<\phi_{t}, c^{\prime}(t)>=0$ e $\left\|\phi_{t}(q)\right\|^{2}=1, \forall q \in S^{n-1}$.

Está claro que, para qualquer $\phi$ que satisfaça as condições (i) e (ii), a imagem de $y$ será a mesma. Além disso, fazendo uma mudança de parâmetro $s=s(t) \operatorname{com} \frac{d s}{d t} \neq 0$, não modificamos a condição $\left|r^{\prime}\right| \leq\left\|c^{\prime}\right\|$ ou a imagem de $y$.

Uma maneira de definir tal $\phi$ pode ser como a seguir. Seja $S^{n} \subset \mathbb{R}^{n+1}$ a $n$-esfera unitária euclidiana e $S^{n-1}=S^{n} \cap\left\{x \in \mathbb{R}^{n+1}: x=\left(x_{1}, \ldots, x_{n}, 0\right)\right\}$. Consideremos a aplicação

$$
t \longmapsto \frac{c^{\prime}(t)}{\left\|c^{\prime}(t)\right\|} \in S^{n} \subset \mathbb{R}^{n+1},
$$

e seja $A(t)$ uma família diferenciável a um parâmetro de transformações ortogonais de $\mathbb{R}^{n+1}$ que satisfaz

$$
A(t) e_{n+1}=\frac{c^{\prime}(t)}{\left\|c^{\prime}(t)\right\|}, \quad t \in(a, b) .
$$

Definimos $\phi: S^{n-1} \times(a, b) \rightarrow \mathbb{R}^{n+1} \operatorname{como} \phi(q, t)=A(t) q$. Então $\phi$ é diferenciável, $\phi_{t}$ é imersão ( mais que isso, é um mergulho), e

$$
<\phi(q, t), c^{\prime}(t)>:=<A(t) q, c^{\prime}(t)>=\left\|c^{\prime}(t)\right\|<A(t) q, A(t) e_{n+1}>=0 \text { e }\left\|\phi_{t}(q)\right\|=1 .
$$

\subsubsection{Considerações sobre o final da demonstração do Lema 2.1.3 e Corolário} 2.1.4.

Lembramos que $M$ é uma hipersuperfície conformemente plana compacta e $U$ é seu conjunto de pontos umbílicos. 
Queremos verificar agora, com um pouco mais de detalhes, que se $D$ é uma componente conexa aberta de $M-U$ então ela é difeomorfa a $S^{n-1} \times(a, b)$.

Para determinarmos o intervalo $(a, b)$, consideremos $V \in \mathcal{X}(D)$ campo unitário normal às folhas da folheação de $D$ e seja $\alpha:(a, b) \rightarrow D$ uma curva integral maximal do campo $V$. Seja ainda $\gamma:(a, b) \rightarrow \mathbb{R}^{n+1}$ a curva dos centros de $\Sigma_{t}^{n-1}$ (folhas da folheação de $D$ ), mais precisamente, $\gamma(t)$ é univocamente determinada por

$$
\|p(t)-\gamma(t)\|^{2}=R^{2}(t), \quad \forall p(t) \in \Sigma_{t}^{n-1}
$$

onde $R(t)$ é o raio de $f\left(\Sigma_{t}^{n-1}\right)$ definido em (2.11). Definimos $\varphi: S^{n-1} \times(a, b) \rightarrow D \subset \mathbb{R}^{n+1}$ por $\varphi(q, t)=\gamma(t)+R \phi(q, t)$. Então, $\varphi$ é da forma de $y$, (2.13), e $y$ é localmente homeomorfo a $D$ (pela Proposição 2.2 .3 a seguir). Como $\varphi$ é diferenciável, $\varphi$ é um difeomorfismo local sobre $D$. Mas por construção $\varphi$ é injetora, logo é um difeomorfismo global sobre $D$ o que finaliza a demonstração do Lema 2.1.5.

Com relação ao Corolário 2.1.4, queremos verificar que se $M$ náo tem pontos umbílicos então $M$ é homeomorfa a $S^{n-1} \times S^{1}$, se $M$ é orientável ou a uma garrafa de Klein generalizada, caso contrário. Observamos que todo ponto de $M$ admite uma vizinhança aberta homeomorfa a $S^{n-1} \times(a, b)$ (chamada vizinhança saturada, veja definição 2.2.4), onde o intervalo $(a, b)$ define a curva dos centros das $(n-1)$-esferas das folheação de $M$ restrita a tal vizinhança. Observamos ainda que esta vizinhança é homeomorfa a um fibrado de $(n-1)$-esferas sobre um intervalo. Este fato, aliado à compacidade de $M$, nos dá que a curva dos centros das $(n-1)$ esferas da folheação de $M$ é fechada. Além disso, tal curva é uma variedade 1-dimensional compacta, logo homeomorfa a $S^{1}$. Concluimos então que $M$ é homeomorfa a um fibrado de $(n-1)$-esferas sobre $S^{1}$, donde vem que $M$ é homeomorfa a $S^{n-1} \times S^{1}$ (se $M$ é orientável) ou a uma "garrafa de Klein generalizada", veja [St] pag. 134.

Veremos agora quais condições $y$ deve satisfazer para ser uma hipersuperfície conformemente plana imersa. Chamaremos $\lambda$ ao autovalor de multiplicidade $(n-1)$ do operador de Weingarten de $y$.

Proposição 2.2.3 Assuma que $y(q, t)=c(t)-S c^{\prime}(t)+R \phi(q, t)$, dada por (2.13), é uma hipersuperfície imersa. Então y é uma hipersuperfície conformemente plana sem pontos umbilicos 
e $\lambda \neq 0, \forall t$. Reciprocamente, qualquer hipersuperficie conformemente plana $f: M^{n} \rightarrow \mathbb{R}^{n+1}$, $n \geq 4$, sem pontos umbilicos e com $\lambda(t) \neq 0 \forall t$, é localmente da forma $y$. Além disso, se $M$ é orientável, $f(M)$ está contido na imagem de uma hipersuperfície da forma $y$.

Demonstração: Primeiro vamos demonstrar a recíproca. Pelas considerações feitas anteriormente, podemos tomar uma vizinhança $W$ de $p \in M$, uma curva $c(t)=f+\lambda^{-1} N$ e uma função positiva $r(t)=\lambda^{-1}$. Derivando $c=f+\lambda^{-1} N$ com relação a $t$, temos

$$
c^{\prime}(t)=\frac{\partial f}{\partial t}+\lambda^{-1} \frac{\partial N}{\partial t}-\frac{\lambda^{\prime}}{\lambda^{2}} N=T+\lambda^{-1} \bar{\nabla}_{T} N-\frac{\lambda^{\prime}}{\lambda^{2}} N=\frac{(\lambda-\mu)}{\lambda} T-\frac{\lambda^{\prime}}{\lambda^{2}} N,
$$

onde $\mu$ é tal que $A_{N} T=\mu T$. Então, tomando $\alpha=\|T\|^{-1}$, temos

$$
\left\|c^{\prime}(t)\right\|^{2}=\frac{(\lambda-\mu)^{2}}{\lambda^{2}}\|T\|^{2}+\left(\frac{\lambda^{\prime}}{\lambda^{2}}\right)^{2}=\frac{(\lambda-\mu)^{2}}{\lambda^{2} \alpha^{2}}+\left(r^{\prime}\right)^{2} .
$$

Como $\lambda-\mu \neq 0$, concluímos que $\left\|c^{\prime}\right\|^{2}>\left(r^{\prime}\right)^{2}$, ou melhor, $\left\|c^{\prime}\right\|>\left|r^{\prime}\right|$. Então, a curva $c(t)$ e a função positiva $r(t)$ satisfazem a condição imposta nos comentários iniciais.

Observemos agora que, no Lema 2.1.3, a compacidade de $M$ foi utilizada apenas para garantir que $\beta \neq 0$, onde $\beta^{2}$ é a curvatura das folhas da folheação de $M$ dada pela distribuição $D_{\lambda}$. Como temos $\lambda \neq 0$, podemos usar o mesmo argumento para concluir que $M$ é folheada por $(n-1)$-esferas $\Sigma^{n-1}$ com raio $\frac{1}{\beta}$ onde

$$
\frac{1}{\beta}=\left(\lambda^{2}+\frac{\lambda^{\prime 2} \alpha^{2}}{(\lambda-\mu)^{2}}\right)^{-\frac{1}{2}}=R \text {. }
$$

De fato,

$$
R=r\left(1-\frac{r^{\prime 2}}{\left\|c^{\prime}\right\|^{2}}\right)^{\frac{1}{2}}=\left(\frac{r^{2}}{\left\|c^{\prime}\right\|^{2}}\left(\left\|c^{\prime}\right\|^{2}-r^{\prime 2}\right)\right)^{\frac{1}{2}}
$$

Mas usando (2.14) vem que

$$
R=\left(\lambda^{2} \frac{\lambda^{2} \alpha^{2}}{(\lambda-\mu)^{2}}\left(\frac{(\lambda-\mu)^{2}}{\lambda^{2} \alpha^{2}}+\frac{\lambda^{\prime 2}}{\lambda^{4}}\right)\right)^{-\frac{1}{2}}=\left(\lambda^{2}+\frac{\alpha^{2} \lambda^{\prime 2}}{(\lambda-\mu)^{2}}\right)^{-\frac{1}{2}}=\frac{1}{\beta} .
$$

Como $c^{\prime}$ está no plano gerado por $T$ e $N$ e o vetor $\xi=\left(\alpha \frac{\lambda^{\prime}}{\lambda-\mu} \alpha T+\lambda N\right) \beta^{-1}$ é tal que $<c^{\prime}, \xi>=0$, temos que $\left\{\frac{c^{\prime}}{\left\|c^{\prime}\right\|}, \xi\right\}$ forma uma base ortonormal para o plano por $T$ e $N$. Então,

$$
N=<N, \frac{c^{\prime}}{\left\|c^{\prime}\right\|}>\frac{c^{\prime}}{\left\|c^{\prime}\right\|}+<N, \xi>\xi
$$


o que, após algumas substituições é equivalente a

$$
N=\frac{1}{r}\left(S c^{\prime}-R \xi\right) .
$$

Como $c(t)=f(q, t)+r(t) N(q, t)$ temos que

$$
f(q, t)=c(t)-r(t) N=c(t)-S c^{\prime}(t)+R \xi(q, t),
$$

ou seja, a restrição $f_{\left.\right|_{W}}$ é localmente da forma $y$. Notemos que, se $M$ é orientável, a curva $c(t)$ e a função positiva $r(t)$ estão definidas em toda a hipersuperfície $M$. Como a mudança de parâmetro para $c(t)$ não modifica a imagem de $y$, tomaremos como parâmetro a função comprimento de arco $s$ e então obteremos uma hipersuperfície $y$ tal que, localmente, a imagem de $f$ está contida na imagem de $y$. Daí, se tomarmos $p \in M$ tal que $U$ e $V$ são vizinhanças de $p$ com $U \neq V, f(U)$ está contido numa hipersuperfície da forma $y$, bem como $f(V)$. Mas, como $U \cap V \neq \emptyset$, temos que $f(U)$ e $f(V)$ estão contidos na mesma hipersuperfície $y$. Tomando uma carta para $M$ notamos que esta propriedade é global, o que finaliza esta parte da demonstração.

Agora, vamos assumir que $y(q, t)=c(t)-S c^{\prime}(t)+R \phi(q, t)$ é uma hipersuperfície imersa. Como $\left\langle c^{\prime}, \phi\right\rangle=0$ então

$$
<y-c, y-c\rangle=\frac{\left(r r^{\prime}\right)^{2}}{\left\|c^{\prime}\right\|^{2}}+r^{2}-\frac{\left(r r^{\prime}\right)^{2}}{\left\|c^{\prime}\right\|^{2}}=r^{2},
$$

portanto, $\|y-c\|^{2}=r^{2}$. Escolhendo coordenadas ortogonais $\left(u_{1}, \ldots, u_{n-1}\right)$ para $S^{n-1}$ e diferenciando $\|y-c\|^{2}=r^{2}$ com relação a $t$ e $u_{i}$ para $i=1,2, \ldots, n-1$, obtemos

$$
\begin{gathered}
<y-c, \frac{\partial y}{\partial t}-c^{\prime}(t)>=r(t) r^{\prime}(t), \\
<y-c, \frac{\partial y}{\partial u_{i}}>=0, \quad i=1,2, \ldots, n-1 .
\end{gathered}
$$

Da expressão de $y$ e do fato de $\left\langle c^{\prime}, \phi\right\rangle=0$ concluímos que $\left\langle y-c, c^{\prime}\right\rangle=-r r^{\prime}$. Isso nos leva, por (2.15) ao seguinte resultado

$$
<y-c, \frac{\partial y}{\partial t}>=0 \text {. }
$$

Segue de (2.16) e (2.17) que $(y-c)$ é normal a $y\left(S^{n-1} \times(a, b)\right)$ logo, existe $N$ normal unitária a $y\left(S^{n-1} \times(a, b)\right)$ tal que $y-c=r N$. Então,

$$
\frac{\partial y}{\partial u_{i}}=r \frac{\partial N}{\partial u_{i}}=r \bar{\nabla}_{V_{i}} N=-r A_{N}\left(V_{i}\right)
$$


ou seja, $V_{i}=-r A_{N}\left(V_{i}\right)$. Concluímos então que $y$ é uma hipersuperfície conformemente plana com $\lambda=-r^{-1}$ como autovalor de $A_{N}$ com multiplicidade (pelo menos) $n-1$.

Para mostrarmos que $y$ é não umbílica vamos provar a existência de $\mu \neq \lambda$ tal que $\mu$ é autovalor de $A_{N}$. Observemos inicialmente que $y\left(S^{n-1} \times(a, b)\right)$ é naturalmente folheada por $(n-1)$-esferas (por construção). Então podemos utilizar um sistema de coordenadas ortogonais $\left(u_{1}, \ldots, u_{n-1}, \tau\right)$ para $y$ adaptado a esta folheação. Pelos comentários iniciais, a aplicação

$$
c(u, \tau)=y+\lambda^{-1} N
$$

é tal que $\frac{\partial c}{\partial u_{i}}=0$, logo $c$ só depende de $\tau$ e, portanto, $y-c(\tau)=-\lambda^{-1} N$ e $y-c(t)=r N=$ $-\lambda^{-1} N$, logo $\left(u_{1}, \ldots u_{n-1}, t\right)$ é um sistema de coordenadas ortogonais pois

$$
\left\|\frac{\partial y}{\partial t}-c^{\prime}(t)\right\|=\left\|\frac{\partial y}{\partial \tau}-\dot{c}(\tau(t))\right\|
$$

Daí, $\frac{\partial}{\partial t}=T$ é tal que $-\bar{\nabla}_{T} N=A_{N}(T)=\mu T$. Além disso, como $\left\|c^{\prime}\right\|^{2}>\left(r^{\prime}\right)^{2}$, e

$$
\left\|c^{\prime}\right\|^{2}=\left\langle\frac{\partial y}{\partial t}-r^{\prime} N-r \frac{\partial N}{\partial t}, \frac{\partial y}{\partial t}-r^{\prime} N-r \frac{\partial N}{\partial t}\right\rangle=\left(r^{\prime}\right)^{2}+\left\|\frac{\partial y}{\partial t}-r \frac{\partial N}{\partial t}\right\|^{2},
$$

deduz-se que $\frac{\partial y}{\partial t}-r \frac{\partial N}{\partial t} \neq 0, \operatorname{logo}, T-\frac{\mu}{\lambda} T \neq 0$, ou seja, $\mu \neq \lambda$.

Definição 2.2.4 Uma hipersuperfície conformemente plana não umbílica $M$ está saturada pela folheação $D_{\lambda}$ se para cada $p \in M$ ela contém o completamento da folha passando por $p$.

Corolário 2.2.5 Seja $f: M^{n} \rightarrow \mathbb{R}^{n+1}, n \geq 4$, uma imersão conformemente plana não umbilica e assuma que $M$ é saturada pela folheação $D_{\lambda}$. Seja $H$ uma vizinhança saturada de uma folha de $D_{\lambda}$, assuma que $\lambda \neq 0$ e $H$ é homeomorfo a $S^{n-1} \times(a, b)$. Então existe uma imersão $y: H \rightarrow \mathbb{R}^{n+1}$ da forma (2.13) tal que $f(H)=y(H)$.

Demonstração: Como $H$ é homeomorfo a $S^{n-1} \times(a, b)=B$ e $B$ é simplesmente conexo, então $H$ é simplesmente conexo, logo $H$ é orientável. Pela Proposição 2.2.3 temos que $f(H) \subset g(B)$ para $g(q, t)=c(t)-S c^{\prime}(t)+R \phi(q, t)$. Mas, se $h: B \rightarrow H$ é o homeomorfismo mencionado então $y=g \circ h^{-1}: H \rightarrow \mathbb{R}^{n+1}$ é tal que $f(H) \subset y(H)=g(B)$. Por outro lado, $y(H) \subset f(H)$ 
pois, como $H$ é saturada, dado $p \in H$ e considerando $\Sigma_{p}$ a $(n-1)$-esfera da folheação de $H$ que passa por $p$, existe $q \in \Sigma_{p}$ tal que $f(q)=y(p)$. Mas, como $q \in \Sigma_{p}$ concluimos que $f(q) \in f\left(\Sigma_{p}\right) \subset f(H)$, logo $y(p) \in f(H)$, ou seja, $y(H) \subset f(H)$.

Um ponto $(q, t)$ da aplicação $y(q, t)$ definida em (2.13) é singular quando a diferencial de $y$ é não injetiva.

Proposição 2.2.6 Seja $y(q, t)=c(t)-S c^{\prime}(t)+R \phi(q, t)$ como em (2.13). Um ponto $(q, t) e^{\prime}$ singular para y se e somente se as duas condiçôes abaixo são satisfeitas

(i) $1-S^{\prime}=\left\|c^{\prime}\right\|^{-2}\left\{R<\phi, c^{\prime \prime}>+S<c^{\prime}, c^{\prime \prime}>\right\}$,

(ii) $R^{\prime}=S\left\langle c^{\prime \prime}, \phi\right\rangle$, onde $R$ e $S$ são definidos em (2.11) e (2.10).

A condição (i) implica a condição (ii) e, se $S \neq 0$, (ii) implica (i). Além disso, (i) pode ser escrita como

$$
\left\|c^{\prime}\right\|^{2}-\left(r^{\prime}\right)^{2}-r r^{\prime \prime}+S<c^{\prime}, c^{\prime \prime}>=R<\phi, c^{\prime \prime}>
$$

Demonstração: Usaremos as coordenadas ortogonais utilizadas na demonstração da Proposição 2.2.3, $\left(u_{1}, . ., u_{n-1}, t\right)$. Então, como $y=c-S c^{\prime}+R \phi$, chamando $\frac{\partial y}{\partial t}$ de $y^{\prime}$ e $\frac{\partial \phi}{\partial t}$ de $\phi^{\prime}$, vem que $y^{\prime}=c^{\prime}-S^{\prime} c^{\prime}-S c^{\prime \prime}+R^{\prime} \phi+R \phi^{\prime}=\left(1-S^{\prime}\right) c^{\prime}-S c^{\prime \prime}+R^{\prime} \phi+R \phi^{\prime}$. Por outro lado, $\frac{\partial y}{\partial u_{i}}=R \frac{\partial \phi}{\partial u_{i}}$ $\mathrm{e}<\frac{\partial y}{\partial u_{i}}, y^{\prime}>=0$ pois são parâmetros ortogonais para $y$, logo $<\frac{\partial \phi}{\partial u_{i}}, y^{\prime}>=0$. Daí ,

$$
0=<\frac{\partial \phi}{\partial u_{i}}, y^{\prime}>=<\frac{\partial \phi}{\partial u_{i}},\left(1-S^{\prime}\right) c^{\prime}-S c^{\prime \prime}+R^{\prime} \phi+R \phi^{\prime}>=-S<c^{\prime \prime}, \frac{\partial \phi}{\partial u_{i}}>+R<\phi^{\prime}, \frac{\partial \phi}{\partial u_{i}}>,
$$

para $i=1,2, \ldots, n-1$.

Segue-se que $<-S c^{\prime \prime}+R \phi^{\prime}, \frac{\partial \phi}{\partial u_{i}}>=0$ ou seja, $-S c^{\prime \prime}+R \phi^{\prime}$ é ortogonal a $\frac{\partial \phi}{\partial u_{i}}$ para todo $i \in\{1,2, \ldots, n-1\}$. Então, $-S c^{\prime \prime}+R \phi^{\prime}$ está no plano gerado por $c^{\prime}$ e $\phi$. Por esse motivo podemos escrever

$$
R \phi^{\prime}-S c^{\prime \prime}=<R \phi^{\prime}-S c^{\prime \prime}, c^{\prime}>\frac{c^{\prime}}{\left\|c^{\prime}\right\|^{2}}+<R \phi^{\prime}-S c^{\prime \prime}, \phi>\phi
$$

Como $\left\langle\phi^{\prime}, \phi>=0 \mathrm{e}\left\langle\phi, c^{\prime}\right\rangle=0\right.$, concluimos que $\left\langle\phi^{\prime}, c^{\prime}\right\rangle=-\left\langle\phi, c^{\prime \prime}\right\rangle$, logo $<R \phi^{\prime}, c^{\prime}>=-R<\phi, c^{\prime \prime}>$ e, portanto,

$$
R \phi^{\prime}-S c^{\prime \prime}=<-R \phi-S c^{\prime}, c^{\prime \prime}>\frac{c^{\prime}}{\left\|c^{\prime}\right\|^{2}}-<S c^{\prime \prime}, \phi>\phi .
$$


Como $y^{\prime}=\left(1-S^{\prime}\right) c^{\prime}-S c^{\prime \prime}+R \phi^{\prime}+R^{\prime} \phi$, temos ainda que

$$
y^{\prime}=\left[\left\|c^{\prime}\right\|^{2}\left(1-S^{\prime}\right)+<-R \phi-S c^{\prime}, c^{\prime \prime}>\right] \frac{c^{\prime}}{\left\|c^{\prime}\right\|^{2}}+\left[R^{\prime}-S<c^{\prime \prime}, \phi>\right] \phi .
$$

Decorre daí que $y^{\prime}=0$ se, e somente se, $\left\|c^{\prime}\right\|^{2}\left(1-S^{\prime}\right)+<-R \phi-S c^{\prime}, c^{\prime \prime}>=0$ e $R^{\prime}-S\left\langle c^{\prime \prime}, \phi\right\rangle=0$, pois $c^{\prime}$ e $\phi$ são linearmente independentes. Portanto,

$$
y^{\prime}=0 \Leftrightarrow\left\{\begin{array}{l}
1-S^{\prime}=\frac{1}{\left\|c^{\prime}\right\|^{2}}\left[S<c^{\prime \prime}, c^{\prime}>+R<\phi, c^{\prime \prime}>\right] \\
R^{\prime}=S<c^{\prime \prime}, \phi>.
\end{array}\right.
$$

Calculando agora as derivadas de $S$ e $R$ temos,

$$
\begin{gathered}
S^{\prime}=\left(\frac{r r^{\prime}}{\left\|c^{\prime}\right\|^{2}}\right)^{\prime}=-2 S \frac{<c^{\prime}, c^{\prime \prime}>}{\left\|c^{\prime}\right\|^{2}}+\frac{\left(\left(r^{\prime}\right)^{2}+r r^{\prime \prime}\right)}{\left\|c^{\prime}\right\|^{2}}, \\
R^{\prime}=\left(r\left(1-\frac{\left(r^{\prime}\right)^{2}}{\left\|c^{\prime}\right\|^{2}}\right)^{\frac{1}{2}}\right)^{\prime}=r^{\prime}\left(1-\frac{\left(r^{\prime}\right)^{2}}{\left\|c^{\prime}\right\|^{2}}\right)^{\frac{1}{2}}+\left(1-\frac{\left(r^{\prime}\right)^{2}}{\left\|c^{\prime}\right\|^{2}}\right)^{-\frac{1}{2}}\left(<c^{\prime}, c^{\prime \prime}>\frac{S r^{\prime}}{\left\|c^{\prime}\right\|^{2}}-S r^{\prime \prime}\right),
\end{gathered}
$$

portanto$$
1-S^{\prime}=\frac{\left\|c^{\prime}\right\|^{2}-\left(r^{\prime}\right)^{2}-r r^{\prime \prime}}{\left\|c^{\prime}\right\|^{2}}+2<c^{\prime}, c^{\prime \prime}>\frac{S}{\left\|c^{\prime}\right\|^{2}}
$$

e, fazendo $R^{\prime}=R^{-1} R R^{\prime}$, concluimos que

$$
R^{\prime}=R^{-1} S\left(\left\|c^{\prime}\right\|^{2}-\left(r^{\prime}\right)^{2}+S<c^{\prime}, c^{\prime \prime}>-r r^{\prime \prime}\right),
$$

donde

$$
R R^{\prime}=S\left(\left\|c^{\prime}\right\|^{2}-\left(r^{\prime}\right)^{2}+S<c^{\prime}, c^{\prime \prime}>-r r^{\prime \prime}\right) .
$$

Segue que, se (i) é satisfeita então

$$
\frac{\left\|c^{\prime}\right\|^{2}-\left(r^{\prime}\right)^{2}-r r^{\prime \prime}}{\left\|c^{\prime}\right\|^{2}}+2<c^{\prime}, c^{\prime \prime}>\frac{S}{\left\|c^{\prime}\right\|^{2}}=1-S^{\prime}=\left\|c^{\prime}\right\|^{-2}\left\{R<\phi, c^{\prime \prime}>+S<c^{\prime}, c^{\prime \prime}>\right\},
$$

portanto $\left\|c^{\prime}\right\|^{2}-\left(r^{\prime}\right)^{2}-r r^{\prime \prime}+S<c^{\prime}, c^{\prime \prime}>=R<\phi, c^{\prime \prime}>$, logo

$$
S\left(\left\|c^{\prime}\right\|^{2}-\left(r^{\prime}\right)^{2}-r r^{\prime \prime}+S<c^{\prime}, c^{\prime \prime}>\right)=S R<\phi, c^{\prime \prime}>.
$$

Por (2.18) temos $R R^{\prime}=S R<\phi, c^{\prime \prime}>$, logo, como $R \neq 0$ a condição (ii) é satisfeita.

Se $S \neq 0$ e (ii) é satisfeita, novamente por (2.18) vem que

$$
S\left(\left\|c^{\prime}\right\|^{2}-\left(r^{\prime}\right)^{2}-r r^{\prime \prime}+S<c^{\prime}, c^{\prime \prime}>\right) R^{-1}=R^{\prime}=S<\phi, c^{\prime \prime}>,
$$


logo

$$
\left\|c^{\prime}\right\|^{2}-\left(r^{\prime}\right)^{2}-r r^{\prime \prime}+S<c^{\prime}, c^{\prime \prime}>=R<\phi, c^{\prime \prime}>
$$

o que nos leva a

$$
\left(1-S^{\prime}\right)\left\|c^{\prime}\right\|^{2}=R<\phi, c^{\prime \prime}>+S<c^{\prime}, c^{\prime \prime}>
$$

Finalmente, como

$$
1-S^{\prime}=\frac{\left\|c^{\prime}\right\|^{2}-\left(r^{\prime}\right)^{2}-r r^{\prime \prime}}{\left\|c^{\prime}\right\|^{2}}+2<c^{\prime}, c^{\prime \prime}>\frac{S}{\left\|c^{\prime}\right\|^{2}}
$$

e

$$
1-S^{\prime}=\left\|c^{\prime}\right\|^{-2}\left\{R<\phi, c^{\prime \prime}>+S<c^{\prime}, c^{\prime \prime}>\right\}
$$

concluimos que

$$
\left\{R<\phi, c^{\prime \prime}>+S<c^{\prime}, c^{\prime \prime}>\right\}=\left\|c^{\prime}\right\|^{2}-\left(r^{\prime}\right)^{2}-r r^{\prime \prime}+2 S<c^{\prime}, c^{\prime \prime}>,
$$

ou seja,

$$
R<\phi, c^{\prime \prime}>=\left\|c^{\prime}\right\|^{2}-\left(r^{\prime}\right)^{2}-r r^{\prime \prime}+S<c^{\prime}, c^{\prime \prime}>.
$$

\subsection{Cirurgia Conforme}

O objetivo desta seção é apresentar um método de se realizar uma cirurgia numa hipersuperfície conformemente plana, de tal forma que a hipersuperfície resultante ainda seja conformemente plana. Para tanto, consideremos $f: M^{n} \rightarrow \mathbb{R}^{n+1}$ uma hipersuperfície conformemente plana e $p \in M$ um ponto não umbílico.

Já foi visto que se $H$ é uma vizinhança saturada de uma folha esférica que passa por $p$, então sua imagem pode ser escrita como $y(H)$ onde $y$ é da forma $y(q, t)=c(t)-S c^{\prime}(t)+R \phi(q, t)$. Definiremos tal $H$ como uma alça conforme ao redor de $p$.

Iremos mostrar que podemos retirar uma pequena alça conforme $H^{\prime} \subset H$ e "fechar" os "buracos esféricos" resultantes de tal forma que a hipersuperfície obtida ainda seja conformemente plana. Esse processo é denominado cirurgia conforme ao longo da folha que passa por $p$. 
Para realizá-lo, consideramos $H$ uma alça conforme e assumimos que a curva $c(t)$ da expressão $y(q, t)=c(t)-S c^{\prime}(t)+R \phi(q, t)$ é tal que $\left\|c^{\prime}\right\|=c t e$. Tomamos um ponto $p$ pertencente à folha correspondente a $t=t_{0}$ e retiramos de $H$ a alça correspondente ao intervalo $\left(t_{0}-2 \varepsilon, t_{0}+2 \varepsilon\right)$, onde $\varepsilon>0$ é pequeno. Em $\left(t_{0}-2 \varepsilon, t_{0}-\varepsilon\right)$ mantemos os valores de $r(t) \mathrm{e}$ $\left\|c^{\prime}\right\|$ e trocamos a curva $c(t)$ de tal forma que, no intervalo $\left(t_{0}-\varepsilon, t_{0}\right)$, ela se torne uma reta. Isto pode ser feito sem a introdução de singularidades, uma vez que a condição da Proposição 2.2.6 se reduz a $\left\|c^{\prime}\right\|^{2}-r^{\prime 2}-r r^{\prime \prime}=R\left\langle\phi, c^{\prime \prime}\right\rangle$, pois $\left\langle c^{\prime}, c^{\prime \prime}\right\rangle=0$ e o processo todo pode ser realizado fazendo $R<\phi, c^{\prime \prime}>$ tender monotonamente para zero. Vamos mostrar que podemos fazer $R \rightarrow 0$ quando $t \rightarrow t_{0}$ sem introduzir singularidades e mantendo a hipersuperfície conformemente plana. Como modificamos y para uma hipersuperfície de rotação próximo de $t_{0}$, certamente devemos ter $R^{\prime} \rightarrow-\infty$ quando $t \rightarrow t_{0}$.

Geometricamente a situação é a observada na figura 2.5 .

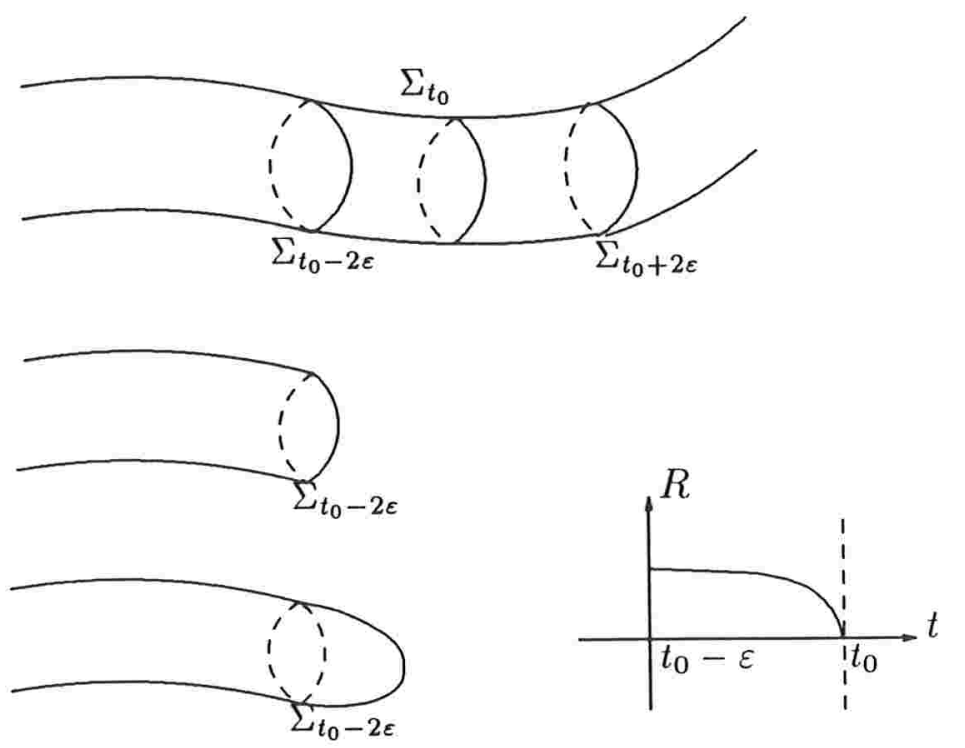

Figura 2.5: esquema geométrico da cirurgia conforme

O lema que se segue nos dá condições para que $R^{\prime} \rightarrow-\infty$ quando $t \rightarrow t_{0}$, ou seja, $R \rightarrow 0$.

Lema 2.3.1 Quando $t \rightarrow t_{0}$, seja $\left\|c^{\prime}\right\| \rightarrow \alpha>0,\left\|c^{\prime}\right\|^{\prime} \rightarrow \gamma \leq 0, r^{\prime} \rightarrow \stackrel{+}{-} \alpha, r \rightarrow \beta>0 e$ $r^{\prime \prime} \rightarrow \delta$. Então $R=r\left(1-\frac{r^{\prime 2}}{\left\|c^{\prime}\right\|^{2}}\right)^{\frac{1}{2}} \rightarrow 0 e$

(i) se $r^{\prime} \rightarrow \alpha e-\delta<|\gamma|$, então $R^{\prime} \rightarrow-\infty$,

(ii) se $r^{\prime} \rightarrow-\alpha$ e $\delta<|\gamma|$, então $R^{\prime} \rightarrow-\infty$. 
Demonstração: Por hipótese temos que

$$
R=r\left(1-\frac{r^{2}}{\left\|c^{\prime}\right\|^{2}}\right)^{\frac{1}{2}} \rightarrow \beta\left(1-\frac{\alpha^{2}}{\alpha^{2}}\right)^{\frac{1}{2}}=0 .
$$

Por outro lado,

$$
R^{\prime}=r^{\prime}\left(1-\frac{r^{\prime 2}}{\left\|c^{\prime}\right\|^{2}}\right)^{\frac{1}{2}}-\frac{r r^{\prime}}{\left\|c^{\prime}\right\|^{2}}\left(1-\frac{r^{\prime 2}}{\left\|c^{\prime}\right\|^{2}}\right)^{-\frac{1}{2}}\left(\frac{r^{\prime \prime}}{\left\|c^{\prime}\right\|}-\frac{r^{\prime}}{\left\|c^{\prime}\right\|} \frac{\left\|c^{\prime}\right\|^{\prime}}{\left\|c^{\prime}\right\|}\right)
$$

e então, quando $t \rightarrow t_{0}$ temos

(i)se $r^{\prime} \rightarrow \alpha$ e $-\delta<|\gamma|$, então $R^{\prime} \rightarrow-\beta\left(\frac{1}{0}\right)\left(\frac{\delta-\gamma}{\alpha}\right) \rightarrow-\infty$

(ii)se $r^{\prime} \rightarrow-\alpha$ e $\delta<|\gamma|$, então $R^{\prime} \rightarrow \beta\left(\frac{1}{0}\right)\left(\frac{\delta+\gamma}{\alpha}\right) \rightarrow-\infty$, donde decorre o lema.

Lema 2.3.2 Seja y dado no intervalo $\left(t_{0}-\varepsilon, t_{0}\right)$ por $y(q, t)=c(t)-S c^{\prime}(t)+R \phi(q, t)$, onde $c(t)$ é uma reta com $\left\|c^{\prime}\right\|=$ cte. Então nós podemos trocar $r(t)$ e $\left\|c^{\prime}\right\|$ tal que $R \rightarrow 0$ quando $t \rightarrow t_{0}$, de tal forma que a hipersuperfície resultante seja conformemente plana e não foram introduzidas singularidades.

Demonstração: O problema encontrado na prova do lema é o controle da ocorrência de singularidades.

Nas condições acima, temos que $S \neq 0$, logo, a Proposição 2.2 .6 nos dá que os pontos singulares de $y$ satisfazem a equação $\left.\left\|c^{\prime}\right\|^{2}-r^{2}-r r^{\prime \prime}+S\left\langle c^{\prime}, c^{\prime \prime}\right\rangle=R<c^{\prime \prime}, \phi\right\rangle$.

Como $\left\|c^{\prime}\right\|$ é constante e queremos verificar o que ocorre quando $R \rightarrow 0$, a expressão passa a ser

$$
A=\left\|c^{\prime}\right\|^{2}-r^{2}-r r^{\prime \prime}=0 .
$$

A partir daqui, a prova seguirá da análise de vários casos.

caso 1: $r^{\prime}>0, r^{\prime \prime}>0,\left\|c^{\prime}\right\|^{2}-r^{2}>r r^{\prime \prime}$, então $A>0$.

Para estudar este caso, vamos dividir o intervalo $\left(t_{0}-\varepsilon, t_{0}\right)$ em quatro partes, denotando-as por (I), (II), (III) e (IV) e mostrar que $A$ não muda de sinal em $\left(t_{0}-\varepsilon, t_{0}\right)$.

Em (I) partindo por $r^{\prime}>0$ e $r^{\prime \prime}>0$, podemos fazer $r^{2}$ crescer mas não atingir $\left\|c^{\prime}\right\|$ e $r^{\prime \prime}$ decrescer monotonamente $\left(r^{\prime \prime \prime}<0\right)$ tal que, no ponto final de (I) tenhamos $r^{\prime \prime}=0$ e $r^{\prime 2}>0$. Então, $A>0 \mathrm{em}$ (I) pois tomamos $\left\|c^{\prime}\right\|=$ cte e tínhamos inicialmente $\left\|c^{\prime}\right\|^{2}-r^{\prime 2}>r r^{\prime \prime}>0$. 
Em (II), continuamos fazendo $r^{\prime \prime}$ decrescer, bem como iniciamos o decrescimento de $r^{\prime 2}$ monotonamente, até termos, no ponto final de (II), $r^{\prime 2}=0$ e $r^{\prime \prime}<0$. Durante este processo tomamos o cuidado de manter $r^{\prime \prime \prime}<0$ mas, no ponto final de (II) fizemos $r^{\prime \prime \prime} \rightarrow 0$. Então, $A^{\prime}=-3 r^{\prime} r^{\prime \prime}-r r^{\prime \prime \prime}>0, \operatorname{logo} A$ é crescente em (II). Como $A>0$ no ponto inicial de (II), concluimos que $A>0$ em (II). Em (III) continuamos decrescendo $r^{\prime}$ tal que, no ponto final de (III) tenhamos $r^{\prime}=-\alpha$ onde $\alpha$ é uma constante positiva menor que $\left\|c^{\prime}\right\|$. Além disso fazemos $r^{\prime \prime}$ crescer, mantendo-a negativa, até que no ponto final de (III) tenhamos $r^{\prime \prime}=0$. Como $A^{\prime}=-3 r^{\prime} r^{\prime \prime}-r r^{\prime \prime \prime}<0$ temos que $A$ é decrescente em (III) de $A=\left\|c^{\prime}\right\|^{2}+r\left|r^{\prime \prime}\right|>0$ para $A=\left\|c^{\prime}\right\|^{2}-r^{\prime 2}>0, \operatorname{logo} A>0$ em (III).

Até aqui mantivemos $\left\|c^{\prime}\right\|$ constante e variamos $r^{\prime}$. Em (IV) mantemos $r^{\prime}=-\alpha$ e fazemos $\left\|c^{\prime}\right\| \rightarrow \alpha>0$ quando $t \rightarrow t_{0}$. Pelo Lema 2.3.1 sabemos que não são introduzidas singularidades em $t=t_{0}$. Vamos mostrar que $A>0$ em (IV).

Notamos que no ponto inicial de (IV) $A>0$ e, da continuidade de $A$, temos que isso se verifica numa vizinhança desse ponto. Então

$$
A^{\prime}=\frac{2\left\|c^{\prime}\right\|^{2}+r^{\prime 2}}{\left\|c^{\prime}\right\|}\left\|c^{\prime}\right\|^{\prime}-\frac{r r^{\prime}}{\left\|c^{\prime}\right\|^{2}}\left(\left\|c^{\prime}\right\|^{\prime}\right)^{2}+\frac{r r^{\prime}}{\left\|c^{\prime}\right\|^{2}}\left\|c^{\prime}\right\|^{\prime \prime}
$$

e podemos fazer $\left\|c^{\prime}\right\|$ decrescer de tal forma que $\left\|c^{\prime}\right\|^{\prime}<0$ e $\left\|c^{\prime}\right\|^{\prime \prime}<0$. Se necessário, podemos ajustar o comprimento de (IV) para que possamos tomar $\left\|c^{\prime}\right\|^{\prime}$ tão grande que $\frac{r r^{\prime}}{\left\|c^{\prime}\right\|^{2}}\left(\left\|c^{\prime}\right\|^{\prime}\right)^{2}$ seja maior que o primeiro fator da soma. Como $r^{\prime}<0$ então teremos $A^{\prime}>0$, portanto $A$ crescente em (IV), o que nos dá $A>0$ em (IV). Veja figura 2.6

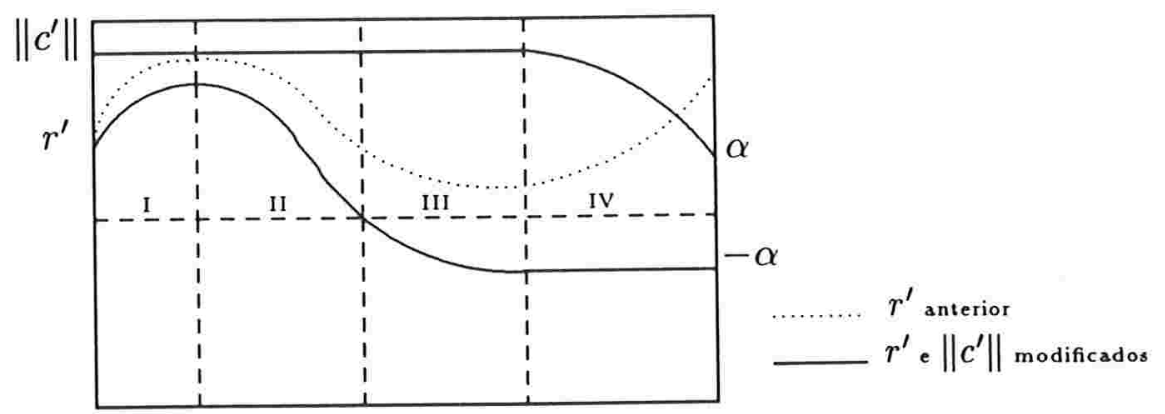

Figura 2.6: caso 1

caso 2: $r^{\prime}>0, r^{\prime \prime}>0,\left\|c^{\prime}\right\|^{2}-r^{\prime 2}<r r^{\prime \prime}$. Então $A<0$.

Se tomarmos inicialmente $r^{\prime \prime \prime}>0$, temos $A^{\prime}=-3 r^{\prime} r^{\prime \prime}-r r^{\prime \prime \prime}<0$ e $A$ decrescente. Basta estudarmos o comportamento de $A$ próximo de $t_{0}$. Fazendo $r^{\prime} \rightarrow\left\|c^{\prime}\right\|=$ cte quando $t \rightarrow t_{0} \mathrm{e}$ 
mantendo $r^{\prime}, r^{\prime \prime}, r^{\prime \prime \prime}$ positivos de tal forma que as condições do Lema 2.3.1 se verifiquem, temos $A<0$ para $t$ próximo de $t_{0}$.

Por outro lado, tomando $r^{\prime \prime \prime}<0$, fazemos $r^{\prime \prime \prime} \rightarrow 0$ mantendo $r^{\prime}$ e $r^{\prime \prime}$ positivos e tais que $A<0$ ( $r^{\prime \prime}$ decrescente mas positivo). A partir daí passamos para $r^{\prime \prime \prime}>0$ e repetimos o argumento anterior.

caso 3: $r^{\prime}<0, r^{\prime \prime}<0,\left\|c^{\prime}\right\|^{2}-r^{\prime 2}>r r^{\prime \prime}$. Então $A>0$.

Tomando inicialmente $r^{\prime \prime \prime}<0$ temos $r^{\prime \prime}$ decrescente e, por hipótese, $A=\left\|c^{\prime}\right\|^{2}-r^{\prime 2}+r\left|r^{\prime \prime}\right|>0$. Basta verificarmos o que acontece próximo de $t_{0}$. Fazendo $r^{\prime} \rightarrow-\left\|c^{\prime}\right\|=c t e$ quando $t \rightarrow t_{0} \mathrm{e}$ mantendo $r^{\prime \prime}$ e $r$ "' negativos de tal forma que as condiçôes do Lema 2.3.1 se verifiquem, temos $A>0$ para $t$ próximo de $t_{0}$. Por outro lado, tomando $r^{\prime \prime \prime}>0$, fazemos $r^{\prime \prime \prime} \rightarrow 0$ mantendo $r^{\prime} \mathrm{e}$ $r^{\prime \prime}$ negativos e tais que $A>0$ ( $r^{\prime \prime}$ crescente mas negativo). A partir daí passamos para $r^{\prime \prime \prime}<0$ e repetimos o argumento anterior.

caso 4: $r^{\prime}<0, r^{\prime \prime}>0,\left\|c^{\prime}\right\|^{2}-r^{\prime 2}>r r^{\prime \prime}$. Então $A>0$.

Iniciamos dividindo o intervalo $\left(t_{0}-\varepsilon, t_{0}\right)$ em duas partes (I) e (II).

Em (I) fazemos $r^{\prime 2}$ decrescer monotonamente $\left(2 r r^{\prime \prime}<0\right)$ de tal forma que, no ponto final de $(\mathrm{I}), r^{\prime} \rightarrow 0$. Observamos que se $r^{\prime \prime \prime}>0,\left(r^{\prime \prime \prime} \rightarrow 0\right.$ no ponto final de (I) $)$ tal que $r^{\prime \prime}$ cresce suavemente, $A$ varia de $\left(\left\|c^{\prime}\right\|^{2}-r^{\prime 2}-r r^{\prime \prime}\right)>0$ para $\left(\left\|c^{\prime}\right\|^{2}-r r^{\prime \prime}\right)$ que ainda é positivo, portanto $A>0$ em (I). Se $r^{\prime \prime \prime}<0, r^{\prime \prime}$ é decrescente mas positivo e observamos que $A^{\prime}>0$, logo $A$ é crescente em (I) ou seja, $A>0$ em (I). Em qualquer das hipóteses, em (II) recaimos no caso 1.

caso 5: $r^{\prime}>0, r^{\prime \prime}<0,\left\|c^{\prime}\right\|^{2}-r^{\prime 2}>r r^{\prime \prime}$. Então $A>0$.

Iniciamos dividindo o intervalo $\left(t_{0}-\varepsilon, t_{0}\right)$ em três partes (I), (II) e (III).

Em (I) fazemos $r^{\prime 2}$ decrescer monotonamente, então $2 r^{\prime} r^{\prime \prime}<0,\left(r^{\prime}>0, r^{\prime \prime}<0\right)$. Podemos fazer isso de tal forma que $r^{\prime \prime}$ decresça $\left(r^{\prime \prime \prime}<0\right)$, com $r^{\prime \prime \prime} \rightarrow 0$ e $r^{\prime} \rightarrow 0$ no ponto final de (I). Então $A^{\prime}=-3 r^{\prime} r^{\prime \prime}-r r^{\prime \prime \prime}>0, \operatorname{logo} A$ crescente em (I), portanto $A>0$ em (I).

Em (II), decrescemos $r^{\prime}$ e fazemos $r^{\prime \prime}$ crescer de tal forma que no ponto final de (II) tenhamos $r^{\prime} \rightarrow-\alpha$ e $r^{\prime \prime} \rightarrow 0$. Daí, $A^{\prime}=-3 r^{\prime} r^{\prime \prime}-r r^{\prime \prime \prime}<0$, ou seja, $A$ é decrescente em (II) de $A=\left\|c^{\prime}\right\|^{2}+r\left|r^{\prime \prime}\right|$ para $A=\left\|c^{\prime}\right\|^{2}-\alpha^{2}>0$ (por hipótese), logo $A>0$ em (II).

Em (III) mantemos $r^{\prime}=-\alpha$ e fazemos $\left\|c^{\prime}\right\| \rightarrow \alpha$ quando $t \rightarrow t_{0}$, tal que tenhamos $\left\|c^{\prime}\right\|^{\prime}<0$ 
$\mathrm{e}\left\|c^{\prime}\right\|^{\prime \prime}<0$. Como

$$
A^{\prime}=\frac{2\left\|c^{\prime}\right\|^{2}+r^{2}}{\left\|c^{\prime}\right\|}\left\|c^{\prime}\right\|^{\prime}-\frac{r r^{\prime}}{\left\|c^{\prime}\right\|^{2}}\left(\left\|c^{\prime}\right\|^{\prime}\right)^{2}+\frac{r r^{\prime}}{\left\|c^{\prime}\right\|^{2}}\left\|c^{\prime}\right\|^{\prime \prime},
$$

podemos modificar o comprimento de (III) a fim de termos o primeiro fator da soma menor que a soma dos outros dois, e daí $A^{\prime}>0$ portanto $A$ crescente. Como $A>0$ numa vizinhança do ponto inicial de (III) então $A>0$ em (III). Veja figura 2.7.

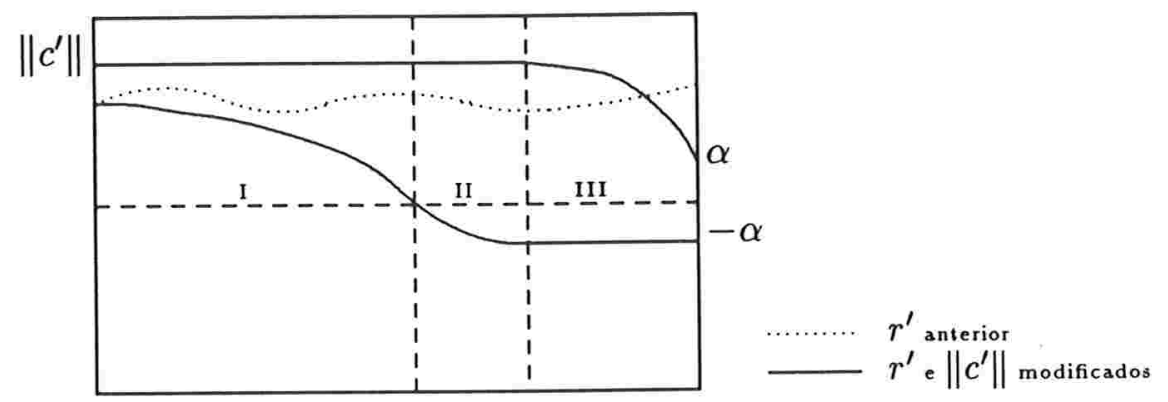

Figura 2.7: caso 5

caso 6: $r^{\prime}<0, r^{\prime \prime}>0,\left\|c^{\prime}\right\|^{2}-r^{\prime 2}<r r^{\prime \prime}$. Então $A<0$.

Iniciamos dividindo o intervalo em duas partes (I) e (II).

Supondo $r^{\prime \prime \prime}>0$ inicialmente, em (I) temos $r^{\prime}<0$ e $r^{\prime \prime}>0$, ambos crescendo. Fazemos $r^{\prime} \rightarrow 0$ e $r^{\prime \prime \prime} \rightarrow 0$ no ponto final de (I) de tal forma que $r^{\prime \prime}$ é grande o suficiente para que $\left\|c^{\prime}\right\|^{2}<r r^{\prime \prime}$. Então $A<0$ em (I). Em (II) temos $r^{\prime}>0, r^{\prime \prime}>0$ e $r^{\prime \prime \prime}<0$, logo recaimos no caso 3 (segunda parte).

Por outro lado, se $r^{\prime \prime \prime}<0$ temos $r^{\prime}<0$ crescendo e $r^{\prime \prime}>0$ decrescendo. Mantemos $r^{\prime}<0$ e $r^{\prime \prime}>0$ tal que $A>0$ e fazemos $r^{\prime \prime \prime} \rightarrow 0$ no ponto final de (I). Em (II) recaimos na hipótese anterior.

Proposição 2.3.3 Seja $f: M^{n} \rightarrow \mathbb{R}^{n+1}, n \geq 4$, uma hipersuperfície conformemente plana compacta e $p \in M$ um ponto não umbilico com $\lambda(p) \neq 0$. Então uma cirurgia conforme pode ser realizada ao longo da folha que passa por $p$.

Demonstração: Como $p$ é um ponto não umbílico e $M$ é localmente folheada por ( $n$-1)-esferas completas, pelo Corolário 2.2.5 existe uma vizinhança $H$ de $p$ tal que $H$ é uma vizinhança 
saturada da folha que passa por $p=\left(p_{0}, t_{0}\right)$ e é homeomorfa a $S^{n-1} \times\left(t_{0}-2 \varepsilon, t_{0}+2 \varepsilon\right)$. Então existe uma imersão $y: H \rightarrow \mathbb{R}^{n+1}$ tal que $y(H)=f(H)$ e $y$ é da forma (2.13).

Pela Proposição 2.2.6 os pontos singulares de $y$ são dados pelos pontos que satisfazem

$$
\begin{gathered}
1-S^{\prime}=\left\|c^{\prime}\right\|^{-2}\left\{R<\phi, c^{\prime \prime}>+S<c^{\prime}, c^{\prime \prime}>\right\}, \\
R^{\prime}=S<c^{\prime \prime}, \phi>
\end{gathered}
$$

e se $S \neq 0$, então basta que satisfaça

$$
\left\|c^{\prime}\right\|-\left(r^{\prime}\right)^{2}-r r^{\prime \prime}+S<c^{\prime}, c^{\prime \prime}>=R<\phi, c^{\prime \prime}>\text {. }
$$

Como $y$ independe da parametrização com relação à variável $t$, podemos tomar uma parametrização para $c(t)$ tal que $\left\|c^{\prime}\right\|=c t e$, ou seja, (2.19) se reduz a $\left\|c^{\prime}\right\|^{2}-\left(r^{\prime}\right)^{2}-r r^{\prime \prime}=R\left\langle\phi, c^{\prime \prime}\right\rangle$.

O processo de cirurgia conforme consiste na modificação da hipersuperfície resultante após a retirada da folha que passa por $p$ trocando-se $c(t)$ por uma reta mantendo $\left\|c^{\prime}\right\|$ constante em $\left(t_{0}-2 \varepsilon, t_{0}\right)$. A partir daí temos uma hipersuperfície de rotação de raio $R$ nessa vizinhança. Então fazemos no intervalo $\left(t_{0}-\varepsilon, t_{0}\right)$ o raio $R$ tender monotonamente para zero quando $t \rightarrow t_{0}$ de tal forma que $R^{\prime} \rightarrow-\infty$, ou seja, sem introduzir singularidades. O Lema 2.3.2 nos diz que isso é possível e que a hipersuperfície resultante é conformemente plana .

\subsection{Caracterização topológica das hipersuperfícies con- formemente planas compactas}

O teorema que se segue é um dos principais deste trabalho.

Teorema 2.4.1 Seja $M^{n}$ uma variedade compacta conexa diferenciável de dimensão $n \geq 4$. Então $M^{n}$ pode ser imersa como uma hipersuperfície conformemente plana $f: M^{n} \rightarrow \tilde{M}^{n+1}(c)$ se, e somente se, $M$ é difeomorfa $a S_{b}^{n}$, onde b é o primeiro número de Betti de $M^{n}$.

Demonstração: Faremos a demonstração para o caso $c=0$. Primeiro vamos demonstrar a recíproca. 
Temos, por hipótese, $M$ uma variedade difeomorfa a $S_{b}^{n}$, onde $S_{b}^{n}$ é uma $n$-esfera com $b$ alças (do tipo $S^{n-1} \times\left(a_{1}, a_{2}\right)$ ) coladas (veja definição na seção 1.4). É fácil ver que existe uma imersão conformemente plana $f: M^{n} \rightarrow \mathbb{R}^{n+1}$ obtida a partir da união de um número conveniente de $n$-esferas em $\mathbb{R}^{n+1}$ utilizando-se alças conformes. Ilustramos na figura 2.8 um exemplo para $b=1$.

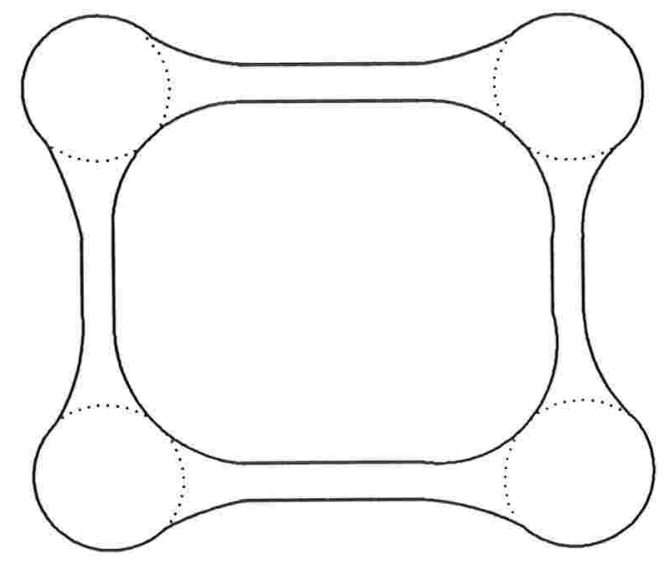

Figura 2.8: imersão conforme de uma variedade difeomorfa a $S_{1}^{n}$

Agora, para demonstrar a primeira implicação, consideremos a hipersuperfície compacta, orientável, conformemente plana e imersa $f: M^{n} \rightarrow \mathbb{R}^{n+1}, n \geq 4$. Seja $\xi \in \mathbb{R}^{n+1}$ tal que $\|\xi\|=1$ e $\xi$ é valor regular da aplicação de Gauss de $f, \phi: M^{n} \rightarrow S^{n}$. Seja $h_{\xi}: M \rightarrow \mathbb{R}$ a função altura de $f$ relativamente a $\xi$, definida por $h_{\xi}(p)=<f(p), \xi>$. Sabemos que num ponto crítico de $h_{\xi}, q \in M$, temos $\xi \perp T_{f(q)}(f(M))$ e que a segunda forma fundamental de $f$ em $q$ é o hessiano de $h_{\xi}$ (veja seção 1.4 , cap. 1).

Como $f$ é conformemente plana, sempre existe um autovalor $\lambda$ da segunda forma fundamental de $f$ com multiplicidade maior ou igual a $(n-1)$ (veja Teorema 2.1.1). Das duas últimas observações concluimos que $h_{\xi}$ tem somente pontos críticos de índices $0,1, n-1$, ou $n$.

Se $h_{\xi}$ só tem pontos críticos de índice 0 e $n$ então segue do teorema de Reeb que $M^{n}$ é homeomorfa a $S^{n}$. Neste caso, $M^{n}$ é simplesmente conexa e, pelo Teorema 1.3.3 (Kuiper), $M^{n}$ é conformemente equivalente a $S^{n}$. Em particular, $M^{n}$ é difeomorfa a $S^{n} \equiv S_{0}^{n}$. 
Por outro lado, se $M^{n}$ não é homeomorfa a $S^{n}$, existe pelo menos um ponto crítico $p$ de $h_{\xi}$ com índice 1 ou $n-1$. Além disso, em tal ponto $\lambda(p) \neq 0$ pois trata-se de ponto não umbílico. Pelos resultados da seção 2.2 , existe uma alça conforme em volta de $p$.

Suponhamos então que $M^{n}$ não é homeomorfa a $S^{n}$ e sejam $p_{1}, \ldots, p_{k}, \quad k \geq 1$, todos os pontos críticos de $h_{\xi}$ com índice 1 ou $n-1$. Sejam $H_{j}, j=1, \ldots, k$, as alças conformes em volta de cada $p_{j}$. Observamos que essa alças são difeomorfas a $S^{n-1} \times(a, b)$. É possível retirar de $M^{n}$ uma alça, eventualmente pequena, $H_{j}^{\prime} \subset H_{j}$ em volta de $p_{j}$, e preencher diferenciavelmente os "buracos esféricos" resultantes com $n$-discos, de modo que o ponto crítico $p_{j}$ seja retirado e que se introduzam, eventualmente, pontos de máximo ou mínimo para $h_{\xi}$. Veja figura 2.9.
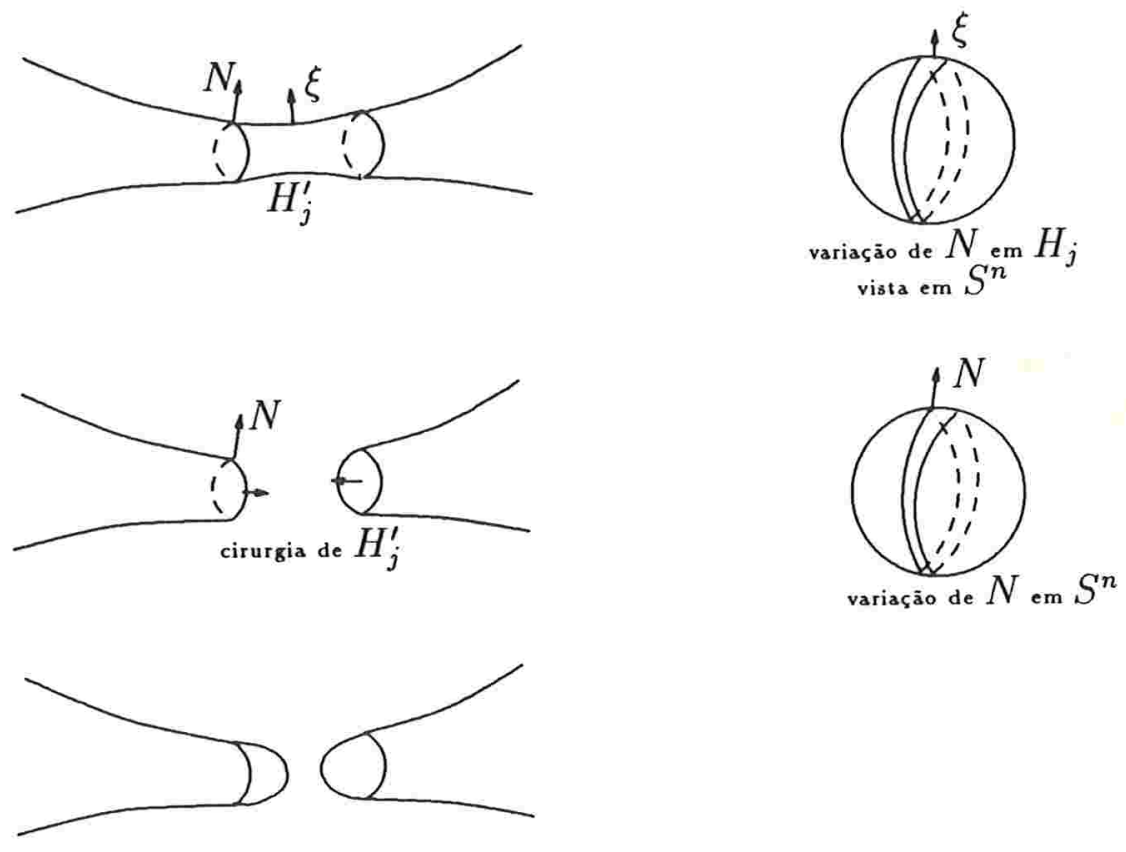

Figura 2.9: cirurgia sem introdução de pontos críticos

Afirmamos que, para pelo menos um índice $j$ do processo acima descrito, não desconectamos $M^{n}$. De fato, se isso ocorresse, depois de efetuado o processo de cirurgia para todos os $p_{j}$ 's teríamos $k+1$ componentes conexas $M_{i}, i=1, \ldots, k+1$. Veja figura 2.10

Como em $M_{i} \quad h_{\xi}$ tem apenas pontos de máximo ou mínimo, cada $M_{i}$ é homeomorfa a $S^{n}$. Por outro lado, como $H_{j}^{\prime}$ desconecta $M$, cada uma das duas componetes conexas de $\partial H_{j}^{\prime}$ estão em componentes conexas distintas de $\cup_{i=1}^{k+1} M_{i}$. Assim, invertendo o processo de cirurgia, 

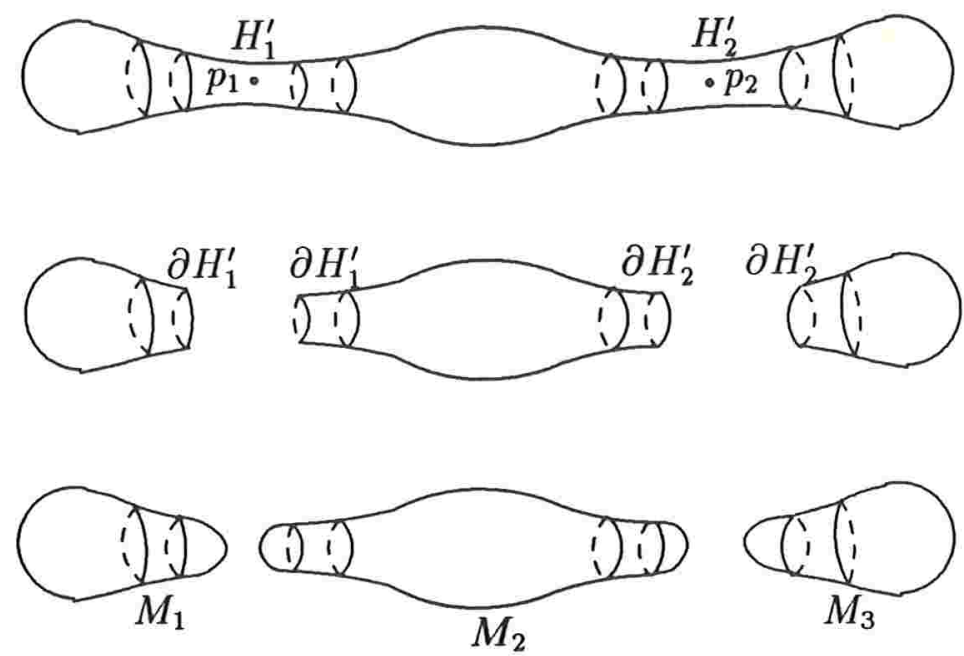

Figura 2.10:

notamos que

$$
M^{n}=M_{1} \sharp M_{2} \sharp \ldots \sharp M_{k+1},
$$

onde $\sharp$ denota a soma conexa. Como cada $M_{i}$ é homeomorfa a $S^{n}$, tem-se $M^{n}$ homeomorfa a $S^{n}$, o que contradiz a nossa suposição acima.

Consideremos $H_{j 1}, \ldots, H_{j b}$ alças cuja omissão, nessa ordem, não desconecte $M$ e apliquemos o processo descrito acima para estas alças. A variedade $M^{\prime}$ obtida ao final do processo é homeomorfa a $S^{n}$. De fato, a retirada de qualquer uma das $(k-b)$ alças restantes desconecta $M^{\prime}$ e, pelo visto acima, $M^{\prime}$ será a soma conexa de $(k-b+1)$ variedades homeomorfas à $S^{n}$.

Seja agora $\tilde{M}$ a variedade obtida a partir de $M^{n}$ após cirurgias conformes sucessivas (veja seção 2.3) nas alças $H_{j 1}, \ldots, H_{j b}$. É claro que $\tilde{f}: \tilde{M} \rightarrow \mathbb{R}^{n+1}, \tilde{f}=f_{\mid \bar{M}}$, é conformemente plana e, como $\tilde{M}$ é obviamente homeomorfa a $M^{\prime}, \tilde{M}$ é homeomorfa a $S^{n}$.

Então, $M^{n}$ é difeomorfa a $S^{n}$ ou $M^{n}$ é obtida a partir de $S_{0}^{n}$ após $b$ etapas, onde cada etapa consiste na retirada do par de discos utilizados para encher os "buracos" na cirurgia conforme e procedendo exatamente como na colagem de alças explicada na seção 1.4. Logo, $M^{n}$ é difeomorfa a $S_{b}^{n}$.

Afirmamos que o número de alças retiradas independe da ordem em que se realizam as cirurgias. Este número é o primeiro número de Betti de $M^{n}$. Para verificar tal fato, utilizaremos 
a sequência de Mayer-Vietoris para os grupos de homologia (onde usaremos coeficientes em $\mathbb{R})$.

Consideremos uma "partição" de $S_{b}^{n}$ por dois abertos $A$ e $B$, onde $A \subset S_{b}^{n}$ é formado pela união (disjunta) das $b$ alças de $S_{b}^{n}\left(A \simeq \bigcup_{j}\left(S^{n-1} \times\left(a_{1 j}, a_{2 j}\right)\right)\right)$, e $B \subset S_{b}^{n}$ é o subconjunto formado a partir de $S^{n}$, retirando-se $2 b n$-discos disjuntos (de raio 1) ( $B \simeq S^{n} \backslash\left(D_{1}^{n} \cup \ldots \cup D_{2 b}^{n}\right)$ ). Então, $A \cap B=S^{n-1} \cup \ldots \cup S^{n-1}, 2 b$ vezes.

Observemos que $B$ e $A \cup B$ são conexos e $A \cap B$ e $A$ tem $2 b$ e $b$ componentes conexas, respectivamente. Logo $H_{0}(A) \simeq \mathbb{R}^{b}, H_{0}(B) \simeq \mathbb{R}, H_{0}(A \cup B) \simeq \mathbb{R}$ e $H_{0}(A \cap B) \simeq \mathbb{R}^{2 b}$.

Da sequência de Mayer-Vietoris,

$$
\begin{aligned}
\ldots \longrightarrow H_{1}(A \cap B) & \longrightarrow H_{1}(A) \oplus H_{1}(B) \longrightarrow H_{1}(A \cup B) \rightarrow \\
& \longrightarrow H_{0}(A \cap B) \longrightarrow H_{0}(A) \oplus H_{0}(B) \longrightarrow H_{0}(A \cup B) \longrightarrow 0
\end{aligned}
$$

que é exata, temos a seguinte correspondência

$$
\ldots \longrightarrow 0 \longrightarrow 0 \oplus 0 \longrightarrow H_{1}(A \cup B) \longrightarrow \mathbb{R}^{2 b} \longrightarrow \mathbb{R}^{b} \oplus \mathbb{R} \longrightarrow \mathbb{R} \longrightarrow 0
$$

donde concluimos que $H_{1}(A \cup B) \simeq \mathbb{R}^{b}$, ou seja, $\operatorname{dim} H_{1}(A \cup B)=b$, o que nos dá $\operatorname{dim} H_{1}\left(M^{n}\right)=$ $b$, logo o primeiro número de Betti de $M^{n}$ é $b$.

Por outro lado, se supusermos que em outra ordem de retirada das alças, tenhamos $M^{n}$ difeomorfa a $S_{d}^{n}$, por procedimento análogo concluimos que $\operatorname{dim} H_{1}(A \cup B)=d$. Sendo assim, $d=b$ e, portanto, o número de alças retiradas independe da ordem em que tal processo ocorre.

Portanto, $M^{n}$ é difeomorfa a $S_{b}^{n}$, onde $b$ é o primeiro número de Betti de $M^{n}$.

\subsection{Observações Finais}

Pela leitura da demonstração do teorema 2.4.1, percebe-se que uma versão um pouco mais fraca do mesmo, a saber: Se $f: M^{n} \rightarrow \bar{M}^{n+1}(c)$ é uma hipersuperfície conformemente plana, compacta, orientável, com $n \geq 4$, então $M^{n}$ é homeomorfa $a S_{b}^{n}$, pode ser provada sem o uso 
de cirurgia conforme e, portanto, não depende do final da seção 2 e da seção 3.

O teorema 2.1.1, essencial em todo este capítulo, não é válido para $n=3$, veja-se [Ca1] e [C-D]. Entretanto, pode-se perceber facilmente que os resultados deste capítulo permanecem válidos, com as mesmas demonstrações, para uma 3-dimensional hipersuperfície conformemente plana onde, em todos os seus pontos, a segunda forma fundamental tem um autovalor de multiplicidade maior ou igual a 2. 


\section{Capítulo 3}

\section{Subvariedades conformemente planas em baixa codimensão}

3.1 Formas bilineares planas e exteriormente ortogonais

Nesta seção $V$ e $W$ denotam espaços vetoriais com dimensão finita $n$ e $r$, respectivamente. Considerando formas bilineares simétricas $\beta: V \times V \rightarrow W$ e $<,>$ : $W \times W \rightarrow \mathbb{R}$ temos as seguintes definições

Definição 3.1.1 A forma bilinear $\beta$ é plana com respeito $a<,>$ se, e somente se, $<\beta(X, Z), \beta(Y, T)>-<\beta(X, T), \beta(Y, Z)>=0, \forall X, Y, Z, T \in V$ 
Neste capítulo, definiremos um produto interno lorentziano $<,>$ para uma base $\left\{\xi_{1}, \ldots, \xi_{r}\right\}$ de $W$ que satisfaz

(i) $<\xi_{i}, \xi_{j}>=0, i \neq j$;

(ii) $\left\langle\xi_{1}, \xi_{1}>=-1 \mathrm{e}<\xi_{i}, \xi_{i}>=1\right.$, para $i=2, \ldots, r$.

Se $<,>$ é um produto interno lorentziano sobre $W$, então $\xi \in W$ é um vetor nulo se $<\xi, \xi>=0$. Não é difícil mostrar que se $\xi$ e $\tilde{\xi}$ são vetores nulos em $W$, então $<\xi, \tilde{\xi}>=0$ se, e somente se, $\xi$ e $\tilde{\xi}$ são linearmente dependentes.

Dada uma forma bilinear simétrica $\beta: V \times V \rightarrow W$ definimos, para cada $X \in V$, a aplicação linear $\beta(X): V \rightarrow W$ por $\beta(X)(Y):=\beta(X, Y)$. É claro que o núcleo e a imagem de $\beta(X)$ são os subespaços

$$
\operatorname{ker} \beta(X)=\{Y \in V: \beta(X, Y)=0\}, \beta(X, V)=\{T \in W: \beta(X, Y)=T, Y \in V\}
$$

de $V$ e $W$, respectivamente. Seja $k=\max _{X \in V} \operatorname{dim} \beta(X, V)$.

Definição 3.1.2 $O$ elemento $X \in V$ é dito regular se $\operatorname{dim} \beta(X, V)=k$. Chamaremos $R E(\beta)=\{X \in V: \operatorname{dim} \beta(X, V)=k\}$ o conjunto dos elementos regulares de $V$.

Para obtermos alguns resultados básicos da teoria das formas bilineares planas, utilizaremos o seguinte

Lema 3.1.3 Suponhamos que $\beta: V \times V \rightarrow W$ seja uma forma bilinear simétrica plana com respeito à forma bilinear simétrica não degenerada $<,>: W \times W \rightarrow \mathbb{R}$. Se $X \in R E(\beta)$ então (i) $<\beta(N, Y), \beta(X, Z)>=0, \forall N \in \operatorname{ker} \beta(X)$ e $Y, Z \in V$;

(ii) $\beta(N, Y) \in \beta(X, V), \forall N \in \operatorname{ker} \beta(X)$ e $Y \in V$.

Demonstração: Sejam $N \in \operatorname{ker} \beta(X)$ e $Y, Z \in V$. Como $\beta$ é simétrica e plana, temos que

$$
0=<\beta(X, N), \beta(Y, Z)>=<\beta(N, X), \beta(Z, Y)>=<\beta(N, Y), \beta(X, Z)>
$$

o que demonstra a parte (i). 
Demonstração: Seja $X \in R E(\beta)$. Afirmamos que $\operatorname{ker} \beta(X)=N(\beta)$. De fato, se $N \in$ $\operatorname{ker} \beta(X)$ e $Y, Z \in V$ temos por (i) do Lema 3.1.3 que $<\beta(N, Y), \beta(X, Z)>=0$, $\operatorname{logo}$ $\beta(N, Y) \in \beta(X, V)^{\perp}$ e, por (ii) do mesmo lema, temos $\beta(N, Y) \in \beta(X, V)$. Como $<,>$ é positivo definido, $\beta(X, V) \cap \beta(X, V)^{\perp}=\{0\}$ e $\beta(N, Y) \in \beta(X, V) \cap \beta(X, V)^{\perp}$. Logo $\beta(N, Y)=$ 0 , ou seja, $\operatorname{ker} \beta(X) \subset N(\beta)$. Mas $N(\beta) \subset \operatorname{ker} \beta(X)$ donde segue a afirmação. Então

$$
\operatorname{dim} N(\beta)=\operatorname{dim} \operatorname{ker} \beta(X)=\operatorname{dim} V-\operatorname{dim} \beta(X, V) \geq \operatorname{dim} V-\operatorname{dim} W .
$$

Proposição 3.1.6 Suponha que $(W,<,>)$ é um espaço com produto interno lorentziano e que $\beta: V \times V \rightarrow W$ é uma forma bilinear simétrica plana com respeito $a<,>$. Se $\operatorname{dim} V>\operatorname{dim} W$ e $\beta(X, X) \neq 0, \forall X \neq 0$, então existe um vetor nulo $\xi \neq 0$ em $W$ e uma forma bilinear simétrica $\varphi: V \times V \rightarrow \mathbb{R}$ tal que $\operatorname{dim} N(\beta-\xi \varphi) \geq \operatorname{dim} V-\operatorname{dim} W+2$.

Demonstração: Seja $X \in R E(\beta)$. Afirmamos que a restrição de $<,>$ a $\beta(X, V)$ é degenerada. De fato, se a restrição não fosse degenerada, valeria (i) do Lema 3.1.3 e teríamos

$$
<\beta(N, Y), \beta(X, Z)>=0, \forall Y, Z \in V, \forall N \in \operatorname{ker} \beta(X)
$$

ou seja, $\beta(N, Y)=0$ e $\operatorname{ker} \beta(X) \subset N(\beta)$ e, portanto, $\operatorname{ker} \beta(X)=N(\beta)$. Mas, pela Proposição $3.1 .5, \operatorname{dim} N(\beta) \geq \operatorname{dim} V-\operatorname{dim} W \geq 1 \mathrm{e}$, por hipótese $N(\beta)=\{0\}$, pois $\beta(X, X) \neq 0, \forall X \neq 0$, o que é uma contradição.

Partindo da afirmação acima, existe um vetor $\xi_{1} \neq 0 \mathrm{em} \beta(X, V) \operatorname{com}\left\langle\xi_{1}, \xi_{1}\right\rangle=0$. Como $(W,<,>)$ é um espaço com produto interno lorentziano, podemos estender $\left\{\xi_{1}\right\}$ à uma base $\left\{\xi_{1}, \ldots, \xi_{r}\right\}$ de $W$ tal que a equação matricial

$$
\left(<\xi_{i}, \xi_{j}>\right)=\left(\begin{array}{ccccc}
0 & 1 & 0 & \ldots & 0 \\
1 & 0 & 0 & \ldots & 0 \\
0 & 0 & 1 & \ldots & 0 \\
\vdots & \vdots & \vdots & \ddots & \vdots \\
0 & 0 & 0 & \ldots & 1
\end{array}\right)
$$


é satisfeita. Pelo Lema 3.1.3 (i), $<\beta(N, Y), \beta(X, Z)>=0, \forall Y, Z \in V, \forall N \in \operatorname{ker} \beta(X)$, donde $\beta(N, Y)$ deve ser um vetor nulo em $\beta(X, V)$. Como $\xi_{1}=\beta(X, Z)$ para algum $Z \in V$ e $\beta$ é simétrica e plana, para $N \in \operatorname{ker} \beta(X)$ e $Y \in V$ temos

$$
<\beta(N, Y), \xi_{1}>=<\beta(Y, Z), \beta(X, N)>=0 .
$$

Mas $<,>$ é lorentziano, logo existe $\lambda \in \mathbb{R}$ tal que $\beta(N, Y)=\lambda \xi_{1}$. Fixado então $N \in \operatorname{ker} \beta(X)$, $N \neq 0$, existe $\lambda_{N} \neq 0$ tal que $\beta(N, N)=\lambda_{N} \xi_{1}$. Usando novamente o fato de $\beta$ ser plana temos, para $Y, Z \in V$, que

$$
<\beta(N, N), \beta(Y, Z)>=<\lambda_{Y} \xi_{1}, \lambda_{Z} \xi_{1}>=0 .
$$

Por outro lado, $<\beta(N, N), \beta(Y, Z)>=\left\langle\lambda_{N} \xi_{1}, \beta(Y, Z)>\right.$, donde $<\xi_{1}, \beta(Y, Z)>=$ $0, \forall Y, Z \in V$. Escrevendo

$$
\beta=\sum_{i=1}^{r} \xi_{i} \varphi_{i}
$$

onde $\varphi_{i}=\left\langle\beta, \xi_{i}>\right.$, se $i=3, \ldots, r$, e $\varphi_{2}=\left\langle\beta, \xi_{1}>, \varphi_{1}=\left\langle\beta, \xi_{2}>\right.\right.$, temos que $<\beta, \xi_{1}>=0$ $\mathrm{e}<\xi_{i}, \xi_{1}>=0$, se $i \neq 2 \mathrm{e}<\xi_{2}, \xi_{1}>=1$, e daí concluimos que $\varphi_{2} \equiv 0$. Sejam $\tilde{W} \subset W$ o subespaço gerado por $\left\{\xi_{3}, \ldots, \xi_{r}\right\}$ e $\tilde{\beta}=\beta-\xi_{1} \varphi_{1}$. Então, $\tilde{\beta}(V \times V)=\sum_{i=3}^{r} \xi_{i} \varphi_{i} \subseteq \tilde{W}$. Como $\beta$ e $\xi_{1} \varphi_{1}$ são formas bilineares planas e $<\beta(Y, Z), \xi_{1} \varphi_{1}(A, B)>=0, \forall Y, Z, A, B \in V$, concluimos que $\tilde{\beta}$ é plana.

A restrição de $<,>$ a $\tilde{W}$ é positiva definida e, pela Proposição 3.1 .5 vem que

$$
\operatorname{dim} N(\tilde{\beta}) \geq \operatorname{dim} V-\operatorname{dim} \tilde{W} .
$$

Para concluir basta escrever $\xi=\xi_{1}$ e $\varphi=\varphi_{1}$.

Sejam $\left\{\eta_{1}, \ldots, \eta_{r}\right\}$ uma base ortonormal qualquer de $W$ e $\beta: V \times V \rightarrow W$ forma bilinear simétrica plana, onde agora $<,>$ é um produto interno positivo definido sobre $W$. Temos a seguinte

Definição 3.1.7 Sejam $\left.\varphi_{i}=<\beta, \eta_{i}\right\rangle$, para $i=1, \ldots, r$, formas bilineares simétricas reais. Como $\beta$ é plana tais formas satisfazem a equação

$$
\sum_{i=1}^{r}\left[\varphi_{i}(X, Z) \varphi_{i}(Y, T)-\varphi_{i}(X, T) \varphi_{i}(Y, Z)\right]=0, \forall X, Y, Z, T \in V,
$$

e são chamadas exteriormente ortogonais. 
A definição acima foi dada por Moore em [Moo1]. Para relacioná-la com a definição dada por Cartan em [Ca2], fixemos uma base $\left\{e_{1}, \ldots, e_{n}\right\}$ de $V$ e observemos que, como cada $\varphi_{i}$ é simétrica,

$$
\varphi_{i}(X, Y)=\frac{1}{2} \sum_{j=1}^{n} y_{j} \frac{\partial \varphi_{i}}{\partial x_{j}}(X), i=1, \ldots, r
$$

onde $X=\sum_{j=1}^{n} x_{j} e_{j}, Y=\sum_{j=1}^{n} y_{j} e_{j}$ e $\frac{\partial \varphi_{i}}{\partial x_{j}}(X)=2 \sum_{k=1}^{n} \varphi_{i}\left(e_{j}, e_{k}\right) x_{k}$ é o funcional linear em $V$ obtido calculando-se formalmente a derivada direcional, na direção $e_{j}$, da forma quadrática $X \rightarrow \varphi_{\mathbf{i}}(X, X)$. Convencionando que

$$
\left[\frac{\partial \varphi_{i}}{\partial x_{j}}, \frac{\partial \varphi_{i}}{\partial x_{k}}\right](X, Y)=\frac{\partial \varphi_{i}}{\partial x_{j}}(X) \frac{\partial \varphi_{i}}{\partial x_{k}}(Y)-\frac{\partial \varphi_{i}}{\partial x_{k}}(X) \frac{\partial \varphi_{i}}{\partial x_{j}}(Y)
$$

obtemos que a relação

$$
\sum_{i=1}^{r}\left[\varphi_{i}(X, Z) \varphi_{i}(Y, T)-\varphi_{i}(X, T) \varphi_{i}(Y, Z)\right]=0
$$

é equivalente à relação

$$
\frac{1}{4} \sum_{j, k=1}^{n} z_{j} t_{k}\left\{\sum_{i=1}^{r}\left[\frac{\partial \varphi_{i}}{\partial x_{j}}, \frac{\partial \varphi_{i}}{\partial x_{k}}\right](X, Y)\right\}=0, \forall X, Y, Z, T \in V
$$

onde $Z=\sum_{j=1}^{n} z_{j} e_{j}$ e $T=\sum_{j=1}^{n} t_{j} e_{j}$. Da definição 3.1.7, concluimos que as formas $\varphi_{i}$ são exteriormente ortogonais se, e somente se,

$$
\sum_{i=1}^{r}\left[\frac{\partial \varphi_{i}}{\partial x_{j}}, \frac{\partial \varphi_{i}}{\partial x_{k}}\right](X, Y)=0, \forall X, Y \in V, 1 \leq j, k \leq n
$$

o que é exatamente a definição que Cartan dá para que as $r$ formas quadráticas, $\varphi_{i}(X, X)$, $1 \leq i \leq r$, sejam exteriormente ortogonais. 


\subsection{Os teoremas de Moore e Moore-Morvan}

Seja $f: M^{n} \rightarrow \mathbb{R}^{n+r}$ uma imersão isométrica e $p \in M^{n}$ um ponto qualquer de $M^{n}$.

Definição 3.2.1 Um subespaço $U \subseteq T_{p} M$ é chamado um subespaço umbílico se existe um vetor normal $\eta \in\left(T_{p} M\right)^{\perp}$ tal que

$$
\alpha(X, Y)=<X, Y>\eta, \forall X \in T_{p} M, Y \in U,
$$

onde $\alpha$ é a segunda forma fundamental de $f$.

Proposição 3.2.2 Seja $f: M^{n} \rightarrow \mathbb{R}^{n+r}$ uma imersão isométrica, $r \leq n-3$. Se $M$ é conformemente plana, para todo $p \in M$ existe um subespaço umbílico $U \subseteq T_{p} M$ tal que $\operatorname{dim} U \geq n-r$.

Demonstração: Seja $p \in M^{n}$ e consideremos a segunda forma fundamental $\alpha: T_{p} M \times T_{p} M \rightarrow$ $\left(T_{p} M\right)^{\perp}$ e a forma bilinear simétrica $L: T_{p} M \times T_{p} M \rightarrow \mathbb{R}$ definida em (1.3), Cap. 1. Chamemos $T_{p} M=V$ e $\left(T_{p} M\right)^{\perp} \oplus \mathbb{R}^{2}=W$.

Vamos definir um produto interno lorentziano $\ll, \gg$ sobre $W$ como

$$
\ll\left(A,\left(s_{1}, t_{1}\right)\right),\left(B,\left(s_{2}, t_{2}\right)\right) \gg=<A, B>+s_{1} t_{2}+s_{2} t_{1}
$$

para $A, B \in\left(T_{p} M\right)^{\perp}, s_{1}, s_{2}, t_{1}, t_{2} \in \mathbb{R}$.

Seja $\beta: V \times V \rightarrow W$ forma bilinear simétrica dada por

$$
\beta(X, Y)=(\alpha(X, Y),<X, Y>,-L(X, Y)) .
$$

Pela equação de Gauss temos

$$
R(X, Y, Z, T)=<\alpha(X, Z), \alpha(Y, T)>-<\alpha(X, T), \alpha(Y, Z)>
$$


$\forall X, Y, Z, T \in T_{p} M$ e, como $M^{n}$ é conformemente plana, temos a relação $R(X, Y, Z, T)=L(Y, Z)<X, T>-L(X, Z)<Y, T>+L(X, T)<Y, Z>-L(Y, T)<X, Z>$

$\forall X, Y, Z, T \in T_{p} M$. De (3.1) e (3.2) concluimos que

$$
\ll \beta(X, Z), \beta(Y, T) \gg-\ll \beta(X, T), \beta(Y, Z) \gg=0, \forall X, Y, Z, T \in T_{p} M,
$$

ou seja, $\beta$ é plana. Como $r \leq n-3$ e $\beta(X, X) \neq(0,0,0), \forall X \neq 0$, pela Proposição 3.1.6 existe um vetor nulo $\xi \neq 0$ em $W$, e uma forma bilinear simétrica $\varphi: V \times V \rightarrow \mathbb{R}$ tal que $\operatorname{dim} N(\beta-\xi \varphi) \geq \operatorname{dim} V-\operatorname{dim} W+2=n-r$. Tomemos $U=N(\beta-\xi \varphi)$. Então, $\forall X \in U, Y \in V$ temos $(\beta-\xi \varphi)(X, Y)=0$, ou seja, $\beta(X, Y)=\xi \varphi(X, Y)$.

Normalizando o vetor $\xi$ tal que $\xi=(\eta,(1, t))$, onde $\eta \in\left(T_{p} M\right)^{\perp}$ e $t \in \mathbb{R}$, teremos para $X \in U$ que

$$
\beta(X, Y)=(\alpha(X, Y),<X, Y>,-L(X, Y)>=(\eta,(1, t)) \varphi(X, Y),
$$

então $\alpha(X, Y)=\eta \varphi(X, Y)=\eta<X, Y>, \forall Y \in V$.

Concluimos então que $U \subseteq T_{p} M$ é o subespaço umbílico procurado.

Um dos principais resultados dessa seção é a seguinte caracterização topológica das subvariedades conformemente planas compactas. Observamos que no caso $r=1$ o teorema abaixo não contém os resultados principais do Capítulo 2, mas ainda é o melhor resultado que se tem nesse sentido.

Teorema 3.2.3 Seja $M^{n}$ uma variedade conformemente plana compacta e $f: M^{n} \rightarrow \mathbb{R}^{n+r}$ uma imersão isométrica, $r \leq n-3$. Então $M^{n}$ tem o tipo homotópico de um $C W$ sem células de dimensão $k$ para $r<k<n-r$. Em particular, para $k$ nessas condições, os grupos de homologia $H_{k}\left(M^{n}, \mathbb{Z}\right)$ são nulos.

Para fazermos uma demonstração completa deste teorema precisaremos de alguns resultados preliminares. São eles o teorema fundamental da teoria de Morse (Teorema 1.4.1) e a seguinte proposição 


\section{CAPÍTULO 3. SUBVARIEDADES CONFORMEMENTE PLANAS EM BAIXA CODIMENSÃO 60}

Proposição 3.2.4 Sejam $M^{n}$ uma variedade $n$-dimensional compacta conformemente plana de $\mathbb{R}^{n+r}$ e $h: M^{n} \rightarrow \mathbb{R}$ uma função altura não degenerada sobre $M^{n}$. Então o índice de $h$ em qualquer ponto crítico é menor ou igual a $r$ ou maior ou igual a $n-r$.

Demonstração: Vimos na demonstração da Proposição 3.2.2 que $\alpha(X, Y)=\eta \varphi(X, Y)=$ $=\eta\left\langle X, Y>, \forall X \in N(\beta-\xi \varphi), Y \in V\right.$, onde $\beta: T_{p} M \times T_{p} M \rightarrow\left(T_{p} M\right)^{\perp} \oplus \mathbb{R}^{2} \mathrm{e}$ $\varphi: T_{p} M \times T_{p} M \rightarrow \mathbb{R}$ são bilineares simétricas e $\beta$ é plana sobre $\ll, \gg$ definido por

$$
\ll\left(A,\left(s_{1}, t_{1}\right)\right),\left(B,\left(s_{2}, t_{2}\right)\right) \gg=<A, B>+s_{1} t_{2}+s_{2} t_{1}
$$

onde $<,>$ é o produto interno canônico e $\xi \in\left(T_{p} M\right)^{\perp} \oplus \mathbb{R}^{2}$ é vetor nulo.

Seja $\zeta \in\left(T_{p} M\right)^{\perp}$ vetor unitário e $h_{\zeta}: M^{n} \rightarrow \mathbb{R}$ a função altura. Então, o hessiano de $h_{\zeta}$ no ponto $p$ está relacionado com a segunda forma fundamental do seguinte modo

$$
\left(d^{2} h_{\zeta}\right)(p)(X, Y)=<\alpha(X, Y), \zeta>_{p}, \forall X, Y \in T_{p} M
$$

Pelo visto acima, temos que

$$
<\alpha(X, Y), \zeta>=<X, Y><\eta, \zeta>, \forall X \in N(\beta-\xi \varphi), Y \in T_{p} M
$$

Se $p$ é ponto crítico não degenerado de $h_{\zeta}$, decorre de (3.3) e (3.4) que

$$
\left(d^{2} h_{\zeta}\right)(p)(X, Y)=\lambda<X, Y>, \forall X \in N(\beta-\xi \varphi), Y \in T_{p} M
$$

Escolhemos então uma base ortonormal $\left\{e_{1}, \ldots, e_{n}\right\}$ para $T_{p} M$ tal que $\left\{e_{r+1}, \ldots, e_{n}\right\}$ estão em $N(\beta-\xi \varphi)$. Analisando a matriz de $\left(d^{2} h_{\zeta}\right)$ no ponto $p$ com relação a esta base temos

$$
\left(d^{2} h_{\zeta}\right)=\left[\begin{array}{c|c}
A & 0 \\
\hline 0 & \lambda I_{n-r}
\end{array}\right],
$$

onde $A$ é uma matriz $r \times r$ e $I_{n-r}$ é matriz identidade. Então, o índice de $h_{\zeta}$ em $p$ é menor ou igual a $r(\lambda>0)$, ou maior ou igual a $n-r(\lambda<0)$.

Demonstração do teorema 3.2.3: Para chegarmos à tese do teorema, observemos que, como $M$ é compacta, o conjunto $M^{c}=\left\{p \in M: h_{\zeta}(p) \leq c\right\}$ é compacto $\forall c \in \mathbb{R}$, onde $\zeta$ é unitário em $\mathbb{R}^{n+r}$ e tal que $h_{\zeta}$ é função de Morse em $M$. Logo, pelo Teorema fundamental da 
teoria de Morse (Teorema 1.4.1), $M^{n}$ tem o tipo homotópico de um $C W$ com uma célula de dimensão $k$ para cada ponto crítico de índice $k$ de $h_{\zeta}$. Pela Proposição 3.2.4, o índice de $h_{\zeta}$ em qualquer ponto crítico é menor ou igual a $r$ ou maior ou igual a $n-r$, donde concluimos que o $C W$ não tem células de dimensão $k$ para $r<k<n-r$. Daí, o grupo gerado pelas $k$-células, $C_{k}(M)$, é nulo para $r<k<n-r$. Considerando a sequência longa juntamente com os operadores bordo $\partial_{i}$

$$
\cdots \longrightarrow C_{n-r}(M) \stackrel{\partial_{n-r}}{\longrightarrow} C_{n-r-1}(M) \stackrel{\partial_{n-r-1}}{\longrightarrow} \cdots \stackrel{\partial_{r+2}}{\longrightarrow} C_{r+1}(M) \stackrel{\partial_{r+1}}{\longrightarrow} C_{r}(M) \longrightarrow \cdots,
$$

observamos que $k e r \partial_{r+1} \subset\{0\}, k e r \partial_{r+2} \subset\{0\}, \ldots, k e r \partial_{n-r-1} \subset\{0\}$, donde

$$
H_{k}(M, \mathbb{Z})=\frac{k e r \partial_{k}}{\operatorname{Im} \partial_{k+1}}=0 \text { para } r<k<n-r .
$$

Passamos agora à descrição e demonstração do Teorema de Moore-Morvan [Moo-Mor].

Definição 3.2.5 Um vetor normal $\xi \in\left(T_{p} M\right)^{\perp}$ é chamado quase umbílico se $A_{\xi}$ tem um autovalor de multiplicidade, no mínimo, $n-1$. A imersão $f: M^{n} \rightarrow \mathbb{R}^{n+r}$ é chamada quase umbílica se, para todo $p \in M$, existe uma base ortonormal $\left\{\xi_{1}, \ldots, \xi_{r}\right\}$ de $\left(T_{p} M\right)^{\perp}$ tal que $\xi_{i}$ é quase umbilico para $i=1, \ldots, r$.

Teorema 3.2.6 Seja $f: M^{n} \rightarrow \mathbb{R}^{n+r}$ uma imersão isométrica com $r \leq \min \{4, n-3\}$. Então $M^{n}$ é conformemente plana se, e somente se, $f$ é quase umbílica.

Demonstração: Iniciaremos demonstrando que se $M$ é conformemente plana, então $f$ é quase umbílica.

Seja $R$ o tensor de curvatura de $M$. Como $M$ é conformemente plana temos a expressão

$$
\begin{aligned}
R(X, Y, Z, T)= & L(X, Z)<Y, T>-L(Y, Z)<X, T>+L(Y, T)<X, Z>+ \\
& -L(X, T)<Y, Z>,
\end{aligned}
$$

$\forall X, Y, Z, T \in T_{p} M$, onde $L$ está definido em (1.3). Usaremos a seguir o seguinte resultado de Moore [Mool] que é um corolário imediato da demonstração da Proposição 3.2.4. 
Proposição 3.2.7 Seja $M^{n}$ uma subvariedade conformemente plana de $\mathbb{R}^{n+r}, r \leq n-3$. Então, para todo $p \in M^{n}$, existe uma base ortonormal de $T_{p} M$ tal que a matriz da segunda forma fundamental é dada por

$$
\left(\alpha\left(e_{i}, e_{j}\right)\right)=\left[\begin{array}{c|c}
A & 0 \\
\hline 0 & \lambda \xi I
\end{array}\right]
$$

onde A é uma matriz $r \times r$ de vetores normais, $\xi \in\left(T_{p} M\right)^{\perp}$ é um vetor normal unitário particular e $\lambda \in \mathbb{R}$.

Consideremos agora a forma bilinear simétrica $\beta: T_{p} M \times T_{p} M \rightarrow\left(T_{p} M\right)^{\perp}$ definida por

$$
\beta(X, Y)=\alpha(X, Y)-\lambda<X, Y>\xi
$$

O lema seguinte constitui um dos principais passos da demonstração do teorema.

Lema 3.2.8 $\beta$ é plana.

Admitindo por enquanto o Lema 3.2.8, temos pela Proposição 3.1.5. que $\operatorname{dim} N(\beta) \geq$ $n-r$, onde $N(\beta)$ é definido em 3.1.4. Seja $\left\{\eta_{1}, \ldots, \eta_{r}\right\}$ base ortonormal qualquer de $\left(T_{p} M\right)^{\perp}$ e considerermos as $r$ formas bilineares simétricas reais $\varphi_{i}=<\beta, \eta_{i}>, i=1, \ldots, r$. Pelo que já vimos, as formas quadráticas associadas que chamaremos também de $\varphi_{1}, \ldots, \varphi_{r}$, são exteriormente ortogonais e podemos aplicar os resultados de Cartan [Ca2].

Chamemos $d=\operatorname{dim} N(\beta)$ e observemos que as $\varphi_{k}^{\prime}$ s acima definidas podem ser expressas em função de $n-d$ variáveis, e não menos do que isso. De fato, seja $\left\{e_{1}, \ldots, e_{n-d}, e_{n-d+1}, \ldots, e_{n}\right\}$ uma base ortonormal de $T_{p} M$ tal que $\left[e_{n-d+1}, \ldots, e_{n}\right]=N(\beta)$. Escrevendo $\varphi_{k}\left(e_{i}, e_{j}\right)=a_{i j}^{k}$, vê-se facilmente que $a_{i j}^{k}=0$ se $i$ e/ou $j>n-d$. Logo,

$$
\varphi_{k}(X, X)=\sum_{i, j=1}^{n-d} a_{i j}^{k} x_{i} x_{j}, \quad X=\sum_{i=1}^{n} x_{i} e_{i}
$$

o que mostra a observação feita. O mesmo tipo de argumento mostra ainda que se as $\varphi_{k}^{\prime} \mathrm{s}$ podem ser expressas em função de $d^{\prime}$ variáveis então $d=\operatorname{dim} N(\beta) \geq n-d^{\prime}$. 
Se $\operatorname{dim} N(\beta)=n-r$, então $\varphi_{1}, \ldots, \varphi_{r}$ não podem ser escritas em função de menos que $r$ variáveis e podemos aplicar o resultado principal da seção 20 de Cartan [Ca2] (veja também [S], vol.V, pag. 202) para concluir que existem $r$ formas quadráticas $\psi_{1}, \ldots, \psi_{r}$ que são quadrados perfeitos e tais que $\varphi_{i}=\sum_{j=1}^{r} a_{i j} \psi_{j}, i=1, \ldots, r$, onde $\left(a_{i j}\right)$ é matriz ortogonal. Tomemos agora $\xi_{1}, \ldots, \xi_{r}$ ortonormais em $\left(T_{p} M\right)^{\perp}$ dados por $\xi_{i}=\sum_{j=1}^{r} a_{j i} \eta_{j}$. Então $\beta_{i}=<\beta, \xi_{i}>$ tem posto 1 e portanto, o núcleo de $\beta_{i}, N\left(\beta_{i}\right)$, tem dimensão $n-1$ para todo $i \in\{1, \ldots, r\}$. Fixado $i \in$ $\{1, \ldots, r\}$, seja $\left\{e_{1}, \ldots, e_{n-1}, e_{n}\right\}$ uma base ortonormal de $T_{p} M$ tal que $\left[e_{1}, \ldots, e_{n-1}\right]=N\left(\beta_{i}\right)$. Então,

$$
\begin{aligned}
A_{\xi_{i}}\left(e_{j}\right) & =\sum_{k=1}^{n}<A_{\xi_{i}}\left(e_{j}\right), e_{k}>e_{k}=\sum_{k=1}^{n}<\alpha\left(e_{j}, e_{k}\right), \xi_{i}>e_{k}= \\
& =\sum_{k=1}^{n} \beta_{i}\left(e_{j}, e_{k}\right) e_{k}+\lambda \delta_{j k}<\xi, \xi_{i}>e_{k} .
\end{aligned}
$$

Como $\beta_{i}\left(e_{j}, e_{k}\right)=0$ para $j, k=1, \ldots, n-1$, temos que

$$
A_{\xi_{i}}\left(e_{j}\right)= \begin{cases}\lambda<\xi, \xi_{i}>e_{j} & \text {, se } j \neq n \\ \left(\lambda<\xi, \xi_{i}>+\beta\left(e_{n}, e_{n}\right)\right) e_{n} & , \text { se } j=n\end{cases}
$$

donde concluimos que $A_{\xi_{i}}$ tem um autovalor de multiplicidade, pelo menos, $n-1$, para $i=$ $1, \ldots, r$.

Se $d=\operatorname{dim} N(\beta)>n-r$, temos que $n-d<r \leq 4$, logo $n-d \leq 3$. Neste caso, as condições do resultado da seção 21 de Cartan [Ca2] são satisfeitas, então existem $r$ formas que são quadrados perfeitos $\psi_{1}, \ldots, \psi_{r}$ e tais que $\varphi_{i}=\sum_{j=1}^{r} a_{i j} \psi_{j},\left(a_{i j}\right)$ é matriz ortogonal. Recaímos no caso anterior, o que encerra esta parte da demonstração.

Para demonstrar a recíproca, tomemos $p \in M$ e $\left\{\xi_{1}, \ldots, \xi_{r}\right\}$ base ortonormal de $\left(T_{p} M\right)^{\perp}$ tal que, cada $\xi_{s}, 1 \leq s \leq r$, é quase umbílico. Fixado $s \in\{1, \ldots, r\}$, seja $\left\{e_{1}^{s}, \ldots, e_{n-1}^{s}, e_{n}^{s}\right\}$ base ortonormal de $T_{p} M$ tal que

$$
A_{\xi_{s}}\left(e_{i}^{s}\right)= \begin{cases}\lambda_{s} e_{i}^{s}, & \text { se } 1 \leq i \leq n-1 \\ \mu_{s} e_{n}^{s}, & \text { se } i=n\end{cases}
$$


$\operatorname{Sejam~} X, Y \in T_{p} M$. Então $X=\sum_{i=1}^{n} x_{i} e_{i}^{s}, Y=\sum_{i=1}^{n} y_{i} e_{i}^{s}, \mathrm{e}$

$$
\begin{aligned}
<\alpha(X, Y), \xi_{s}> & =<A_{\xi_{s}}(X), Y> \\
& =<\lambda_{s}\left(\sum_{i=1}^{n-1} x_{i} e_{i}^{s}\right)+\mu_{s} x_{n} e_{n}, \sum_{i=1}^{n} y_{i} e_{i}^{s}> \\
& =\lambda_{s} \sum_{i=1}^{n-1} x_{i} y_{i}+\mu_{s} x_{n} y_{n} \\
& \left.\left.=\lambda_{s}<X, Y>+\left(\mu_{s}-\lambda_{s}\right)<X, e_{n}^{s}\right\rangle<Y, e_{n}^{s}\right\rangle .
\end{aligned}
$$

Tomando $X, Y \in T_{p} M$ tal que $X \perp Y$ e $\|X\|=\|Y\|=1$ temos, pela equação de Gauss, que

$$
k(X, Y)=<\alpha(X, X), \alpha(Y, Y)>-\|\alpha(X, Y)\|^{2} .
$$

Calculando $\alpha(X, X)$ e $\alpha(Y, Y)$ temos

$$
\begin{aligned}
& \alpha(X, X)=\sum_{s=1}^{r}<\alpha(X, X), \xi_{s}>\xi_{s}=\sum_{s=1}^{r}\left[\lambda_{s}+\left(\mu_{s}-\lambda_{s}\right)<X, e_{n}^{s}>^{2}\right] \xi_{s} \\
& \alpha(Y, Y)=\sum_{s=1}^{r}<\alpha(Y, Y), \xi_{s}>\xi_{s}=\sum_{s=1}^{r}\left[\lambda_{s}+\left(\mu_{s}-\lambda_{s}\right)<Y, e_{n}^{s}>^{2}\right] \xi_{s}
\end{aligned}
$$

donde vem que $\%$

$$
\begin{aligned}
k(X, Y)= & <\sum_{s=1}^{r}\left[\lambda_{s}+\left(\mu_{s}-\lambda_{s}\right)<X, e_{n}^{s}>^{2}\right] \xi_{s}, \sum_{s=1}^{r}\left[\lambda_{s}+\left(\mu_{s}-\lambda_{s}\right)<Y, e_{n}^{s}>^{2}\right] \xi_{s}>+ \\
& -\sum_{s=1}^{r}\left(\mu_{s}-\lambda_{s}\right)^{2}<X, e_{n}^{s}>^{2}<Y, e_{n}^{s}>^{2} \\
= & \sum_{s=1}^{r} \lambda_{s}^{2}+\lambda_{s}\left(\mu_{s}-\lambda_{s}\right)\left[<X, e_{n}^{s}>^{2}+<Y, e_{n}^{s}>^{2}\right] .
\end{aligned}
$$

Como $n \geq 4$, sejam $X_{1}, X_{2}, X_{3}, X_{4}$ vetores ortonormais de $T_{p} M$. Então

$$
k\left(X_{1}, X_{2}\right)+k\left(X_{3}, X_{4}\right)=\sum_{s=1}^{r} 2 \lambda_{s}^{2}+\lambda_{s}\left(\mu_{s}-\lambda_{s}\right)\left[\sum_{i=1}^{4}<X_{i}, e_{n}^{s}>^{2}\right]
$$

e

$$
k\left(X_{1}, X_{3}\right)+k\left(X_{2}, X_{4}\right)=\sum_{s=1}^{r} 2 \lambda_{s}^{2}+\lambda_{s}\left(\mu_{s}-\lambda_{s}\right)\left[\sum_{i=1}^{4}<X_{i}, e_{n}^{s}>^{2}\right],
$$

ou seja, $k\left(X_{1}, X_{2}\right)+k\left(X_{3}, X_{4}\right)=k\left(X_{1}, X_{3}\right)+k\left(X_{2}, X_{4}\right)$.

Pelo Teorema 1.3.7 concluimos que $M$ é conformemente plana o que encerra a demonstração. 
Sejam $X, Y \in T_{p} M$. Então $X=\sum_{i=1}^{n} x_{i} e_{i}^{s}, Y=\sum_{i=1}^{n} y_{i} e_{i}^{s}, \mathrm{e}$

$$
\begin{aligned}
<\alpha(X, Y), \xi_{s}> & =<A_{\xi_{o}}(X), Y> \\
& =<\lambda_{s}\left(\sum_{i=1}^{n-1} x_{i} e_{i}^{s}\right)+\mu_{s} x_{n} e_{n}, \sum_{i=1}^{n} y_{i} e_{i}^{s}> \\
& =\lambda_{s} \sum_{i=1}^{n-1} x_{i} y_{i}+\mu_{s} x_{n} y_{n} \\
& =\lambda_{s}<X, Y>+\left(\mu_{s}-\lambda_{s}\right)<X, e_{n}^{s}><Y, e_{n}^{s}>.
\end{aligned}
$$

Tomando $X, Y \in T_{p} M$ tal que $X \perp Y$ e $\|X\|=\|Y\|=1$ temos, pela equação de Gauss, que

$$
k(X, Y)=<\alpha(X, X), \alpha(Y, Y)>-\|\alpha(X, Y)\|^{2} .
$$

Calculando $\alpha(X, X)$ e $\alpha(Y, Y)$ temos

$$
\begin{aligned}
& \alpha(X, X)=\sum_{s=1}^{r}<\alpha(X, X), \xi_{s}>\xi_{s}=\sum_{s=1}^{r}\left[\lambda_{s}+\left(\mu_{s}-\lambda_{s}\right)<X, e_{n}^{s}>^{2}\right] \xi_{s}, \\
& \alpha(Y, Y)=\sum_{s=1}^{r}<\alpha(Y, Y), \xi_{s}>\xi_{s}=\sum_{s=1}^{r}\left[\lambda_{s}+\left(\mu_{s}-\lambda_{s}\right)<Y, e_{n}^{s}>^{2}\right] \xi_{s},
\end{aligned}
$$

donde vem que $\%$

$$
\begin{aligned}
k(X, Y)= & <\sum_{s=1}^{r}\left[\lambda_{s}+\left(\mu_{s}-\lambda_{s}\right)<X, e_{n}^{s}>^{2}\right] \xi_{s}, \sum_{s=1}^{r}\left[\lambda_{s}+\left(\mu_{s}-\lambda_{s}\right)<Y, e_{n}^{s}>^{2}\right] \xi_{s}>+ \\
& -\sum_{s=1}^{r}\left(\mu_{s}-\lambda_{s}\right)^{2}<X, e_{n}^{s}>^{2}<Y, e_{n}^{s}>^{2} \\
= & \sum_{s=1}^{r} \lambda_{s}^{2}+\lambda_{s}\left(\mu_{s}-\lambda_{s}\right)\left[<X, e_{n}^{s}>^{2}+<Y, e_{n}^{s}>^{2}\right] .
\end{aligned}
$$

Como $n \geq 4$, sejam $X_{1}, X_{2}, X_{3}, X_{4}$ vetores ortonormais de $T_{p} M$. Então

$$
k\left(X_{1}, X_{2}\right)+k\left(X_{3}, X_{4}\right)=\sum_{s=1}^{r} 2 \lambda_{s}^{2}+\lambda_{s}\left(\mu_{s}-\lambda_{s}\right)\left[\sum_{i=1}^{4}<X_{i}, e_{n}^{s}>^{2}\right],
$$

e

$$
k\left(X_{1}, X_{3}\right)+k\left(X_{2}, X_{4}\right)=\sum_{s=1}^{r} 2 \lambda_{s}^{2}+\lambda_{s}\left(\mu_{s}-\lambda_{s}\right)\left[\sum_{i=1}^{4}<X_{i}, e_{n}^{s}>^{2}\right],
$$

ou seja, $k\left(X_{1}, X_{2}\right)+k\left(X_{3}, X_{4}\right)=k\left(X_{1}, X_{3}\right)+k\left(X_{2}, X_{4}\right)$.

Pelo Teorema 1.3.7 concluimos que $M$ é conformemente plana o que encerra a demonstração. 
Para finalizar este capítulo, apresentamos agora a

Demonstração do Lema 3.2.8: Iremos mostrar que

$$
<\beta(X, Z), \beta(Y, W)>-<\beta(X, W), \beta(Y, Z)>=0, \forall X, Y, Z, W \in T_{p} M
$$

Seja $V \subset T_{p} M$ o subespaço gerado por $\left\{e_{1}, \ldots, e_{r}\right\}$ e $V^{\perp}$ o subespaço gerado por $\left\{e_{r+1}, \ldots, e_{n}\right\}$, onde $\left\{e_{1}, \ldots, e_{r}, e_{r+1}, \ldots, e_{n}\right\}$ é a base ortonormal da Proposição 3.2.7. Afirmamos que

$$
L(X, Y)=\frac{1}{2} \lambda^{2}<X, Y>, \forall X \in T_{p} M \forall Y \in V^{\perp} .
$$

De fato, basta mostrarmos a relação para $X$ e $Y$ em $\left\{e_{1}, \ldots, e_{n}\right\}$.

Quando $X=e_{i}, Y=e_{j} \operatorname{com} i \neq j, \quad i \leq r<j$, decorre da equação de Gauss e da Proposição 3.2 .7 que

$$
\begin{aligned}
L\left(e_{i}, e_{j}\right) & =\frac{1}{n-2} \operatorname{Ricc}\left(e_{i}, e_{j}\right)=\frac{1}{n-2} \sum_{k=1}^{n} R\left(e_{k}, e_{i}, e_{j}, e_{k}\right)= \\
& =\frac{1}{n-2} \sum_{k=1}^{n}<\alpha\left(e_{i}, e_{j}\right), \alpha\left(e_{k}, e_{k}\right)>-<\alpha\left(e_{i}, e_{k}\right), \alpha\left(e_{k}, e_{j}\right)>=0 .
\end{aligned}
$$

Quando $X=e_{i}, Y=e_{j}$ com $i \neq j>r$, concluimos por procedimento análogo que $L\left(e_{i}, e_{j}\right)=0$.

Quando $X=Y=e_{i}$ e $i>r$, vamos mostrar que $L\left(e_{i}, e_{i}\right)=\frac{\lambda^{2}}{2}$. Como $n \geq r+3$, existem pelo menos três índices distintos $i, j, l$ tais que $i, j, l>r$. Temos pela Proposição 3.2.7. e por $k(X, Y)=L(X, X)+L(Y, Y)($ veja Teorema 1.3.7), que

$$
\begin{aligned}
& \lambda^{2}=k\left(e_{i}, e_{j}\right)=L\left(e_{i}, e_{i}\right)+L\left(e_{j}, e_{j}\right) \\
& \lambda^{2}=k\left(e_{i}, e_{l}\right)=L\left(e_{i}, e_{i}\right)+L\left(e_{l}, e_{l}\right) \\
& \lambda^{2}=k\left(e_{l}, e_{j}\right)=L\left(e_{l}, e_{l}\right)+L\left(e_{j}, e_{j}\right) .
\end{aligned}
$$

Fazendo $(3.5)+(3.6)$ - (3.7) nas equações acima, obtemos a relação desejada e terminamos a demonstração da afirmação. Observamos agora que, como $V^{\perp} \subset T_{p} M$ é um subespaço umbílico e utilizando a Proposição 3.2.7 temos que $\alpha(X, Y)=\lambda<X, Y>\xi, \forall X \in T_{p} M, \forall Y \in V^{\perp}$. Então, $\langle\alpha(X, Y), \xi\rangle=\lambda\langle X, Y\rangle, \forall X \in T_{p} M, \forall Y \in V^{\perp}$. Logo a relação

$$
\mathrm{L}(X, Y)-\lambda<\alpha(X, Y), \xi>+\frac{1}{2} \lambda^{2}<X, Y>=0, \forall X \in T_{p} M \forall Y \in V^{\perp},
$$


é verdadeira. Daí, dados dois vetores unitários $X \in V$ e $Y \in V^{\perp}$ temos

$$
k(X, Y)=\left\langle\alpha(X, X), \alpha(Y, Y)>-\|\alpha(X, Y)\|^{2}=\lambda<\alpha(X, X), \xi>\right.
$$

$\mathrm{e}$

$$
k(X, Y)=L(X, X)+L(Y, Y)=L(X, X)+\frac{1}{2} \lambda^{2},
$$

donde deduzimos que

$$
L(X, X)-\lambda<\alpha(X, X), \xi>+\frac{1}{2} \lambda^{2}<X, X>=0, \forall X \in V .
$$

Isso mostra que a forma quadrática $Q(X)=B(X, X)$ associada à forma bilinear simétrica $B(X, Y)=L(X, Y)-\lambda\left\langle\alpha(X, Y), \xi>+\frac{1}{2} \lambda^{2}<X, Y>\right.$ é identicamente nula, donde $B \equiv 0$ em $T_{p} M$, isto é, (3.8) vale para qualquer $X, Y \in T_{p} M$.

Agora, se tomarmos $X, Y$ em $T_{p} M$, ortonormais, teremos

$$
\begin{aligned}
& <\beta(X, X), \beta(Y, Y)>-<\beta(X, Y), \beta(X, Y)>= \\
& =k(X, Y)-\lambda<\alpha(X, X), \xi>+\frac{\lambda^{2}}{2}-\lambda<\alpha(Y, Y), \xi>+\frac{\lambda^{2}}{2}= \\
& =L(X, X)-\lambda<\alpha(X, X), \xi>+\frac{1}{2} \lambda^{2}+L(Y, Y)-\lambda<\alpha(Y, Y), \xi>+\frac{1}{2} \lambda^{2}=0 .
\end{aligned}
$$

Consideremos a aplicação 4-linear $\psi: T_{p} M \times T_{p} M \times T_{p} M \times T_{p} M \rightarrow \mathbb{R}$ dada por

$$
\psi(X, Y, Z, W)=<\beta(X, Z), \beta(Y, W)>-<\beta(X, W), \beta(Y, Z)>.
$$

Esta aplicação satisfaz as condições

(i) $\psi(X, Y, Z, W)+\psi(Y, Z, X, W)+\psi(Z, X, Y, W)=0$;

(ii) $\psi(X, Y, Z, W)=-\psi(Y, X, Z, W)$;

(iii) $\psi(X, Y, Z, W)=-\psi(Y, X, W, Z)$;

(iv) $\psi(X, Y, Z, W)=\psi(Z, W, X, Y)$.

Como $\psi(X, Y, X, Y)=0, \forall X, Y \in T_{p} M$ tais que $X \perp Y$ e $\|X\|=\|Y\|=1$, concluimos que $\psi \equiv 0$, ou seja, $\beta$ é plana. 


\section{Referências Bibliográficas}

[A-D ] A. C. Asperti, M. Dajczer: Conformally flat Riemannian manifolds as hipersurfaces of the light cone, Canadian Math. Bull., (1989)

[B ] A. Barros: Hipersuperfícies Conformemente Planas, Monografia de Mestrado (UFC), Fortaleza, Ce, ago/83.

[C ] M. do Carmo: Geometria Riemanniana, IMPA, Rio de Janeiro, 1988, 2 ed.

[C-D ] M. do Carmo, M. Dajczer: General conformally flat hipersurfaces of $\mathbb{R}^{n+1}$, relatório técnico, IMPA, série A-008-junho/82.

[C-D-M ] M. do Carmo, M. Dajczer, F. Mercuri: Compact conformally flat hipersurfaces in a conformally flat Riemannian manifold, Trans. Am. Math. Soc.,288(1985), 189-203.

[Ca1] E. Cartan: La déformation des hypersurfaces dans l'espace conform réel a $n \geq 5$ dimensions, Bull. Soc. Math. France, 45(1917), 57-121.

[Ca2] E. Cartan: Sur les variétés de courbure constante d'un espace euclidien ou noneuclidien, Bull. Soc. Math. France, 48(1920), 132-208.

[D ] M. Dajczer: Submanifolds and isometric immersions, Publish or Perish, Inc., Houston, 1990.

[E ] L.P. Eisenhart: Riemannian Geometry, University Press, Princeton, 1964.

[G ] M.J. Greenberg: Lectures on Algebraic Topology, W.A. Benjamin, Inc., Massachusetts, 1967.

[K ] N. Kuiper: On conformally flat spaces in the large, Ann. Math, 50(1949), 916-924.

[Kul-1 ] R. S. Kulkarni: Curvature and metric, Ann. of Math., 91(1970), 311-331. 
[Kul-2 ] R. S. Kulkarni: Curvature structures and conformal transformations, J. Diff. Geom., $4(1970), 425-451$.

[Ma ] W. S. Massey: Algebraic Topology: An Introduction, Springer-Verlag

[M] F. Mercuri: Conformally Flat Immersions, Note di Matematica, Vol IX, (1989), 85-99.

[Mil] J. Milnor: Morse Theory, Princeton University Press, Princeton, 1973.

[Moo1] J. D. Moore: Conformally flat submanifolds of Euclidean spaces, Math. Ann., 225(1977), 89-97.

[Moo2 ] J. D. Moore: Submanifolds of constant positive curvature I, Duke Math. J., 44(1977), 449-483.

[Moo-Mor] J. D. Moore, J-M. Morvan: Sous-variétés conformemént plates de codimension quatre, C. R. Acad. Sc. Paris, 287(1978), 655A-657A.

[N-Md] S. Nishikawa, Y. Maeda: Conformally flat hipersurfaces in a conformally flat Riemannian manifold, Tôhoku Math. J., 26(1974), 159-168.

[O'N ] B. O'Neill: Semi-Riemannian Geometry, Academic Press, New York, 1983.

[Re ] H. Reckziegel: Completness of curvature surfaces of an isometric immersion, J. Diff. Geom., 14(1979), 7-20.

[Ro ] L. Rodriguêz: Geometria das subvariedades, IMPA, Rio de Janeiro, 1976.

[Ry ] P. Ryan: Homogeneity and some curvature conditions for hipersurfaces, Tôhoku Math. J., 21(1969), 363-388.

[S ] M. Spivak: A Comprehensive Introduction to Differential Geometry, Publish or Perish, Inc., Berkley, 1979.

[St ] N. Steenrod: Topology of Fiber Bundles, Princeton University Press, Princeton, 1951. 